NBER WORKING PAPER SERIES

FISCAL MULTIPLIERS:

LIQUIDITY TRAPS AND CURRENCY UNIONS

Emmanuel Farhi

Iván Werning

Working Paper 18381

http://www.nber.org/papers/w18381

\author{
NATIONAL BUREAU OF ECONOMIC RESEARCH \\ 1050 Massachusetts Avenue \\ Cambridge, MA 02138 \\ September 2012
}

The views expressed herein are those of the authors and do not necessarily reflect the views of the National Bureau of Economic Research.

NBER working papers are circulated for discussion and comment purposes. They have not been peerreviewed or been subject to the review by the NBER Board of Directors that accompanies official NBER publications.

(C) 2012 by Emmanuel Farhi and Iván Werning. All rights reserved. Short sections of text, not to exceed two paragraphs, may be quoted without explicit permission provided that full credit, including $\odot$ notice, is given to the source. 
Fiscal Multipliers: Liquidity Traps and Currency Unions

Emmanuel Farhi and Iván Werning

NBER Working Paper No. 18381

September 2012

JEL No. E52,E62,F41

\section{ABSTRACT}

We provide explicit solutions for government spending multipliers during a liquidity trap and within a fixed exchange regime using standard closed and open-economy models. We confirm the potential for large multipliers during liquidity traps. For a currency union, we show that self-financed multipliers are small, always below unity. However, outside transfers or windfalls can generate larger responses in out- put, whether or not they are spent by the government. Our solutions are relevant for local and national multipliers, providing insight into the economic mechanisms at work as well as the testable implications of these models.

Emmanuel Farhi

Harvard University

Department of Economics

Littauer Center

Cambridge, MA 02138

and NBER

efarhi@harvard.edu

Iván Werning

Department of Economics

MIT

50 Memorial Drive, E51-251a

Cambridge, MA 02142-1347

and NBER

iwerning@mit.edu 


\title{
Fiscal Multipliers: Liquidity Traps and Currency Unions
}

\author{
Emmanuel Farhi \\ Harvard University
}

\author{
Iván Werning \\ MIT
}

August 2012

\begin{abstract}
We provide explicit solutions for government spending multipliers during a liquidity trap and within a fixed exchange regime using standard closed and open-economy models. We confirm the potential for large multipliers during liquidity traps. For a currency union, we show that self-financed multipliers are small, always below unity. However, outside transfers or windfalls can generate larger responses in output, whether or not they are spent by the government. Our solutions are relevant for local and national multipliers, providing insight into the economic mechanisms at work as well as the testable implications of these models.
\end{abstract}

\section{Introduction}

Economists generally agree that macroeconomic stabilization should be handled first and foremost by monetary policy. Yet monetary policy can run into constraints that impair its effectiveness. For example, the economy may find itself in a liquidity trap, where interest rates hit zero, preventing further reductions in the interest rate. Similarly, countries that belong to currency unions, or states within a country, do not have the option of an independent monetary policy. Some economists advocate for fiscal policy to fill this void, increasing government spending to stimulate the economy. Others disagree, and the issue remains deeply controversial, as evidenced by vigorous debates on the magnitude of fiscal multipliers. No doubt, this situation stems partly from the lack of definitive empirical evidence, but, in our view, the absence of clear theoretical benchmarks also plays an important role. Although various recent contributions have substantially furthered our understanding, to date, the implications of standard macroeconomic models have not been fully worked out. This is the goal of our paper. 
We solve for the response of the economy to changes in the path for government spending during liquidity traps or within currency unions using standard closed and open-economy monetary models. A number of features distinguish our approach and contribution. First, our approach departs from existing literature by focusing on fiscal multipliers that encapsulate the effects of spending for any path for government spending, instead of solving for a particular multiplier associated with the expansion of a single benchmark path for spending. Doing so provides insight into the economic mechanism for the effects and delivers results on the varying effectiveness in the timing-back loading versus front loading-for stimulus plans. Second, we obtain simple closed-form solutions for these multipliers. These help uncover the precise mechanisms underlying the effects of fiscal policy and allow us to deliver several new results. Third, we investigate a sharp contrast between the liquidity trap and currency union cases. We isolate the crucial role that outside transfers can play and we compute the corresponding transfer multipliers. Finally, we consider non-Ricardian effects by studying a version of the model with liquidity constrained consumers. This extension may be especially relevant for the recent downturn, which is widely associated with a sharp disruption in financial and credit markets, followed by a slow recovery featuring household deleveraging. Throughout, we draw implications for recent empirical studies on national and local multipliers.

Our results confirm that fiscal policy can be especially potent during a liquidity trap. The multiplier for output is greater than one. The mechanism for this result is that government spending promotes inflation. With fixed nominal interest rates, this reduces real interest rates which increases current spending. The increase in consumption in turn leads to more inflation, creating a feedback loop. The fiscal multiplier is increasing in the degree of price flexibility, which is intuitive given that the mechanism relies on the response of inflation. We show that backloading spending leads to larger effects; the rationale is that inflation then has more time to affect spending decisions.

In a currency union, by contrast, government spending is less effective at increasing output. We show that consumption is depressed, so that the multiplier is less than one. Moreover, price flexibility diminishes the effectiveness of spending, instead of increasing it. We explain this result using a simple argument that illustrates its robustness. Government spending leads to inflation in domestically produced goods and this loss in competitiveness depresses private spending. Applied to current debates in Europe, this highlights a possible tradeoff: putting off fiscal consolidation may postpone internal devaluations that actually help reactivate private spending.

It may seem surprising that fiscal multipliers are necessarily less than one whenever the exchange rate is fixed, because this contrasts sharply with the effects during liquidity 
traps. Our analytical approach allows us to uncover the crucial difference in monetary policy: although a fixed exchange rate implies a fixed nominal interest rate, the converse is not true. Indeed, we prove that the liquidity trap analysis implicitly combines a shock to government spending with a one-off devaluation. The positive response of consumption relies entirely on this devaluation. A currency union rules out such devaluations, explaining the negative response of consumption.

In the context of a currency union, our results uncover the importance of transfers from the outside, from other countries or regions. In the short run, when prices haven't fully adjusted, positive transfers from the rest of the world increase the demand for home goods, stimulating output. We compute "transfer multipliers" that capture the response of the economy to transfers from the outside. We show that these multipliers may be large and depend crucially on the degree of openness of the domestic economy.

Outside transfers are often tied to government spending. In the United States federal military spending allocated to a particular state is financed by the country as a whole. The same is true for exogenous differences in stimulus payments, due to idiosyncratic provisions in the law. Likewise, idiosyncratic portfolio returns accruing to a particular state's coffers represent a windfall for this state against the rest. When changes in spending are financed by such outside transfers, the associated multipliers are a combination of self-financed multipliers and transfer multipliers. As a result, multipliers may be substantially larger than one.

Finally, we explore non-Ricardian effects from fiscal policy by introducing hand-tomouth consumers. We think of this as a tractable way of modeling liquidity constraints. In both in a liquidity trap and in a currency union, government spending now has an additional stimulative effect. It increases the income and consumption of hand-to-mouth agents. This effects is largest when spending is deficit financed; indeed, the effects may in some cases depend entirely on deficits, not spending per se. Overall, although hand to mouth consumers introduce an additional effect most of our conclusions, such as the comparison of fiscal multipliers in a liquidity trap and a currency union, are unaffected.

Related literature. Our paper is related to several strands of theoretical and empirical literatures. We will discuss those that are most closely related.

We contribute to the literature that studies fiscal policy in the New Keynesian model in liquidity traps. Eggertsson (2011), Woodford (2011), Christiano et al. (2011), show that fiscal multipliers can be large at the zero lower bound, while Werning (2012) studies optimal government spending with and without commitment to monetary policy. Gali and Monacelli (2008) study optimal fiscal policy in a currency union, but they conduct an 
exclusively normative analysis and do not compute fiscal multipliers. The results and simulations reported in Corsetti et al. (2011), Nakamura and Steinsson (2011), and Erceg and Linde (2012) show that fiscal multipliers are generally below one under fixed exchange rates yet higher than under flexible exchange rates (away from the zero bound), somewhat validating the conventional Mundell-Flemming view that fiscal policy is more effective with fixed exchange rates (see e.g. Dornbusch, 1980). Our solutions extend these results and help sharpen the intuition for them, by discussing the role of implicit devaluations and transfers. Gali, Lopez-Salido and Valles (2007) introduce hand-to-mouth consumers and study the effects of government spending under a Taylor rule in a closed economy. Our setup extends such an analysis to liquidity traps and currency unions in an open economy.

Our paper is also related to a large empirical literature on fiscal multipliers. Estimating national fiscal multipliers poses serious empirical challenges. The main difficulties arise from the endogeneity of government spending, the formation of expectations about future tax and spending policies, and the reaction of monetary policy. Most of the literature tries to resolve these difficulties by resorting to Structural VARs. Some papers use military spending as an instrument for government spending. The relevant empirical literature is very large, so we refer the reader to Ramey (2011) for a recent survey. Estimating fiscal multipliers in liquidity traps is nearly impossible because liquidity traps are rare. The closest substitute is provided by estimates that condition of the level of economic activity. Some authors (see e.g. Gordon and Krenn, 2010; Auerbach and Gorodnichenko, 2012) estimate substantially larger national multipliers during deep recessions, but the magnitude of these differential effects remains debated (see e.g. Barro and Redlick, 2009).

States or regions within a country offer an attractive alternative with plausible exogenous variations in spending. Indeed the literature on local multipliers has recently been very active, with contributions by Clemens and Miran (2010), Cohen et al. (2010), Serrato and Wingender (2010), Shoag (2010), Acconcia et al. (2011), Chodorow-Reich et al. (2011), Fishback and Kachanovskaya (2010) and Nakamura and Steinsson (2011). These papers tend to find large multipliers. Our paper helps interpret these findings. Government spending at the local level in these experiments is generally tied to transfers from outside. It follows that these estimates may be interpreted as combining spending and transfer multipliers, as we define them here. 


\section{A Closed Economy}

We adopt a continuous time framework. This is convenient for some calculations but is completely inessential to any of our results.

We consider a one-time shock to the current and future path of spending that is realized at the beginning of time $t=0$ that upsets the steady state. To simplify and focus on the impulse response to this shock, we abstract from ongoing uncertainty at other dates. ${ }^{1}$

The remainder of this section spells out the standard New Keynesian model assumptions; readers familiar with this setting may wish to skip directly to Section 3.

Households. There is a representative household with preferences represented by the utility function

$$
\int_{0}^{\infty} e^{-\rho t}\left[\frac{C_{t}^{1-\sigma}}{1-\sigma}+\chi \frac{G_{t}^{1-\sigma}}{1-\sigma}-\frac{N_{t}^{1+\phi}}{1+\phi}\right] d t,
$$

where $N_{t}$ is labor, and $C_{t}$ is a consumption index defined by

$$
C_{t}=\left(\int_{0}^{1} C_{t}(j)^{\frac{\epsilon-1}{\epsilon}} d j\right)^{\frac{\epsilon}{\epsilon-1}}
$$

where $j \in[0,1]$ denotes an individual good variety. Thus, $\epsilon$ is the elasticity between varieties produced within a given country.

Households seek to maximize their utility subject to the budget constraints

$$
\dot{D}_{t}=i_{t} D_{t}-\int_{0}^{1} P_{t}(j) C_{t}(j) d j+W_{t} N_{t}+\Pi_{t}+T_{t}
$$

for $t \geq 0$ together with a no-Ponzi condition. In this equation, $P_{t}(j)$ is the price of domestic variety $j, W_{t}$ is the nominal wage, $\Pi_{t}$ represents nominal profits and $T_{t}$ is a nominal lump sum transfer. The bond holdings of home agents are denoted by $D_{t}$ and the nominal interest rate for the currency union is denoted by $i_{t}$.

Government. Government consumption $G_{t}$ is an aggregate of varieties just as private consumption,

$$
G_{t}=\left(\int_{0}^{1} G_{t}(j)^{\frac{\epsilon-1}{\epsilon}} d j\right)^{\frac{\epsilon}{\epsilon-1}} .
$$

\footnotetext{
${ }^{1}$ Since we are interested in a first order approximation of the equilibrium response to shocks, which can be solved by studying the log-linearized model, the presence of ongoing uncertainty would not affect any of our calculation or conclusions (we have certainty equivalence).
} 
For any level of expenditure $\int_{0}^{1} P_{H, t}(j) G_{t}(j) d j$, the government splits its expenditure across these varieties to maximize $G_{t}$. Spending is financed by lump-sum taxes. Ricardian equivalence holds, so that the the timing of these taxes is irrelevant.

Firms. A typical firm produces a differentiated good with a linear technology

$$
Y_{t}(j)=A_{t} N_{t}(j)
$$

where $A_{t}$ is productivity in the home country.

We allow for a constant employment tax $1+\tau^{L}$, so that real marginal cost deflated is given by $\frac{1+\tau^{L}}{A_{t}} \frac{W_{t}}{P_{t}}$. We take this employment tax to be constant in our model. The tax rate is set to offset the monopoly distortion so that $\tau^{L}=-\frac{1}{\varepsilon}$.

We adopt the standard Calvo price-setting framework. In every moment a randomly flow $\rho_{\delta}$ of firms can reset their prices. Those firms that reset choose a reset price $P_{t}^{r}$ to solve

$$
\max _{P_{t}^{r}} \int_{0}^{\infty} e^{-\rho_{\delta} s-\int_{0}^{s} i_{t+z} d z}\left(P_{t}^{r} Y_{t+s \mid t}-\left(1+\tau^{L}\right) W_{t} \frac{Y_{t+s \mid t}}{A_{t}}\right)
$$

where $Y_{t+k \mid t}=\left(\frac{P_{t}^{r}}{P_{t+k}}\right)^{-\epsilon} Y_{t+k}$, taking the sequences for $W_{t}, Y_{t}$ and $P_{t}$ as given.

\subsection{Equilibrium Conditions}

We now summarize equilibrium conditions for the home country. Market clearing in the goods and labor market

$$
\begin{aligned}
Y_{t} & =C_{t}+G_{t}, \\
N_{t} & =\frac{Y_{t}}{A_{t}} \Delta_{t},
\end{aligned}
$$

where $\Delta_{t}$ is an index of price dispersion $\Delta_{t}=\int_{0}^{1}\left(\frac{P_{H, t}(j)}{P_{H, t}}\right)^{-\epsilon}$. The Euler equation

$$
\sigma \frac{\dot{C_{t}}}{C_{t}}=i_{t}-\pi_{t}-\rho
$$

ensures the agents' intertemporal optimization, where $\pi_{t}=\dot{P}_{t} / P_{t}$ is inflation.

The natural allocation is a reference allocation that prevails if prices are flexible and government consumption is held constant at its steady state value G. We denote the natural allocation with a bar over variables. 
We omit the first-order conditions for the price-setting problem faced by firms here. We shall only analyze a log-linearized version of the model which collapses these equilibrium conditions into the New Keynesian Phillips curve presented below.

\section{National Multipliers in a Liquidity Trap}

To obtain multipliers we study the log-linearized equilibrium conditions around the natural allocation. Define

$$
\begin{gathered}
c_{t}=(1-\mathcal{G})\left(\log \left(C_{t}\right)-\log \left(\bar{C}_{t}\right)\right) \approx \frac{C_{t}-\bar{C}_{t}}{Y} \\
y_{t}=\log Y_{t}-\log \bar{Y}_{t} \approx \frac{Y_{t}-\bar{Y}_{t}}{Y} \quad g_{t}=\mathcal{G}\left(\log G_{t}-\log G\right) \approx \frac{G_{t}-G}{Y}
\end{gathered}
$$

where $\mathcal{G}=\frac{G}{Y}$. So that we have, up to a first order approximation,

$$
y_{t}=c_{t}+g_{t}
$$

The log linearized system is then

$$
\begin{aligned}
\dot{c}_{t} & =\hat{\sigma}^{-1}\left(i_{t}-\pi_{t}-\bar{r}_{t}\right), \\
\dot{\pi}_{t} & =\rho \pi_{t}-\kappa\left(c_{t}+(1-\xi) g_{t}\right),
\end{aligned}
$$

where $\hat{\sigma}=\frac{\sigma}{1-\mathcal{G}}, \lambda=\rho_{\delta}\left(\rho+\rho_{\delta}\right), \kappa=\lambda(\hat{\sigma}+\phi)$ and $\xi=\frac{\hat{\sigma}}{\hat{\sigma}+\phi}$. Equation (1) is the Euler equation and equation (2) is the New Keynesian Philips curve. Here $\bar{r}_{t}$ is the natural rate of interest, defined as the real interest rate that prevail at the natural allocation, i.e. equation (1) with $c_{t}=0$ for all $t \geq 0$ implies $i_{t}-\pi_{t}=\bar{r}_{t}$ for all $t \geq 0$.

It will prove useful to define the following two numbers $v$ and $\bar{v}$ (the eigenvalues of the system):

$$
v=\frac{\rho-\sqrt{\rho^{2}+4 \kappa \hat{\sigma}^{-1}}}{2} \quad \bar{v}=\frac{\rho+\sqrt{\rho^{2}+4 \kappa \hat{\sigma}^{-1}}}{2} .
$$

If prices were completely flexible, then consumption and labor are determined in every period by two static conditions: the labor consumption condition and the resource constraint. Spending affects the solution and gives rise to the neoclassical multiplier $1-\xi$, which is positive but less than 1 and entirely due to a wealth effect on labor supply.

From now on we take as given a path for the interest rate $\left\{i_{t}\right\}$ summarizing monetary policy. To resolve or sidestep issues of multiplicity one can assume that there is a date 
$T$ such that $c_{t}=g_{t}=\pi_{t}=0$ and $i_{t}=\bar{r}_{t}$ for $t \geq T$. $^{2}$ A leading example is a liquidity trap scenario where $i_{t}=0$ and $\bar{r}_{t}<0$ for $t<T$. However, although this is a useful interpretation but is not required for the analysis below.

Remark 1. Suppose $c_{T}=0$ for some date $T$, then

$$
c_{t}=\int_{t}^{T}\left(i_{t+s}-\pi_{t+s}-\bar{r}_{t+s}\right) d s
$$

so that given the inflation path $\left\{\pi_{t}\right\}$ the consumption path $\left\{c_{t}\right\}$ is independent of the spending path $\left\{g_{t}\right\}$.

This remark highlights that the mechanism by which government spending affects consumption, in the New Keynesian model, is inflation which affects the real interest rate. One can draw two implications from this. First, other policy instruments that affect inflation, such as taxes, may have similarly policy effects. Second, empirical work on fiscal multipliers have not focused on the role inflation plays and it may be interesting to test the predicted connection between output and inflation present in New Keynesian models.

\subsection{Fiscal Multipliers Solved}

Since the system is linear it admits a closed form solution. We can express any solution with government spending as

$$
\begin{aligned}
& c_{t}=\tilde{c}_{t}+\int_{0}^{\infty} \alpha_{s}^{c} g_{t+s} d s, \\
& \pi_{t}=\tilde{\pi}_{t}+\int_{0}^{\infty} \alpha_{s}^{\pi} g_{t+s} d s,
\end{aligned}
$$

where $\left\{\tilde{c}_{t}, \tilde{\pi}_{t}\right\}$ are equilibria with $g_{t}=0$ for all $t$. We focus on the integral term $\int_{0}^{\infty} \alpha_{s}^{i} g_{t+s} d s$ for $i=c, \pi$ as a measure of the effects of fiscal policy $g \neq 0$. We assume the integrals are well defined, although we allow and discuss the case where it is $+\infty$ or $-\infty$ below.

Focusing on consumption, we call the sequence of coefficients $\left\{\alpha_{s}^{c}\right\}$ fiscal multipliers. Note that they do not depend on calendar time $t$, nor do they depend on the interest rate paths $\left\{i_{t}\right\}$ and $\left\{r_{t}\right\}$. Thus, the impact on consumption or output, given by the

\footnotetext{
${ }^{2}$ Note that $T$ may be arbitrarily large and will have no impact on the solution provided below. Indeed, the characterization of the equilibrium is valid even without selecting an equilibrium this way: one just interprets $c^{*}$ and $\pi^{*}$ below any equilibrium in the set of equilibrium attained when $g_{t}=0$ for all $t$. The solution then describes the entire set of equilibria for other spending paths $\left\{g_{t}\right\}$.
} 
term $\int_{0}^{\infty} \alpha_{s}^{c} g_{t+s} d s$, depends only on the future path for spending summarized weighted by $\left\{\alpha_{s}^{c}\right\}$.

There are two motivations for adopting $\int_{0}^{\infty} \alpha_{s}^{c} g_{t+s} d s$ as a measure of the impact of fiscal policy, one more practical the other more conceptual:

1. The more practical motivation applies if the economy finds itself in a liquidity trap where interest rates immobilized at zero, at least for some time. Fiscal multipliers $\left\{\alpha_{s}^{c}\right\}$ can then be used to predict the effects of fiscal policy. To see this, suppose the zero lower bound is binding until $T$ so that $i_{t}=0$ for $t<T$; suppose that after $T$ monetary policy delivers an equilibrium with zero inflation, so that $\pi_{t}=0$ for $t \geq T$. As is well known, the resulting equilibrium without government spending ( $g_{t}=0$ for all $t$ ) features a negative consumption gap and deflation: $\tilde{c}_{t}, \tilde{\pi}_{t}<0$ for $t<T$ (e.g. see Werning, 2012).

Now, consider a stimulus plan that attempts to improve this outcome by setting $g_{t}>0$ for $t<T$ and $g_{t}=0$. Then $\int_{0}^{\infty} \alpha_{s}^{c} g_{t+s} d s=\int_{0}^{T} \alpha_{s}^{c} g_{t+s} d s$ is precisely the effect of the fiscal expansion on consumption $c_{t}$, relative to the outcome without the stimulus plan $\tilde{c}_{t}$.

More generally, suppose after the trap we spending may be nonzero and that monetary may or may not be described as securing zero inflation. Even in this case, we may still use fiscal multipliers to measure the impact of fiscal policy during the liquidity trap: one can write $c_{t}=c_{T}+\int_{0}^{T-t} \alpha_{s}^{c} g_{t+s} d s$ for $t<T$, where the $c_{T}$ encapsulates the combined effects of fiscal and monetary policy after the trap $t \geq T$.

2. More conceptually, our fiscal multipliers provide a natural decomposition of the effects of the fiscal policy, over what is attainable by monetary policy alone.

Equations (3a)-(3b) characterize the entire set of equilibria for $g \neq 0$ by providing a one-to-one mapping between equilibria with $g=0$. Both $\tilde{c}_{t}$ and $\tilde{\pi}_{t}$ are equilibria with $g=0$ and are affected by monetary policy, as summarized, among other things, by the interest rate path $\left\{i_{t}\right\}$.

We can represent these facts as a relationship between the set of equilibria with and without government spending,

$$
\mathcal{E}_{g}=\mathcal{E}_{0}+\alpha \cdot g
$$

where $\mathcal{E}_{0}$ represents the set of equilibria when $g_{t}=0$ for all $t$, while $\mathcal{E}_{g}$ is the set of equilibria for a given path for spending $g=\left\{g_{t}\right\}$. Here $\alpha=\left\{\alpha_{s}^{c}, \alpha_{s}^{\pi}\right\}$ collects the 


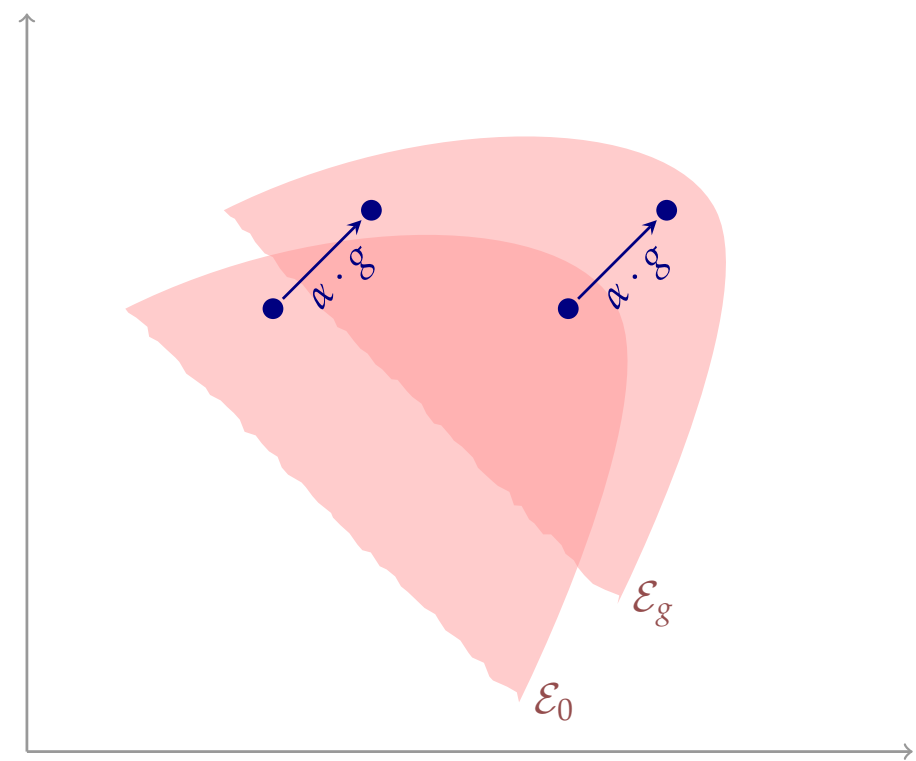

Figure 1: A schematic depiction of the set of equilibria without government spending and the set of equilibria for a given spending path $\left\{g_{t}\right\}$.

fiscal multipliers and the cross product $\alpha \cdot g$ represents the integrals $\int_{0}^{\infty} \alpha_{s}^{i} g_{t+s} d s$ for $i=c, \pi$. The set $\mathcal{E}_{g}$ is a displaced version of $\mathcal{E}_{0}$ in the direction $\alpha \cdot g$. Each equilibrium point in $\mathcal{E}_{0}$ is shifted in parallel by $\alpha \cdot g$ to another equilibrium point in $\mathcal{E}_{g}$ and it shares the same nominal interest rate path $\left\{i_{t}\right\}$. This last fact is unimportant for this second conceptual motivation, since the focus is on comparing the two sets, not equilibrium points. Instead, the important issue is that $\alpha \cdot g$ measures the influence of government spending on the set of equilibria. This provides a conceptual motivation for studying the multipliers $\alpha$, since they summarize this influence. In other words, without spending one can view monetary policy as selecting from the set $\mathcal{E}_{0}$, while with government spending monetary policy can choose from $\mathcal{E}_{\mathcal{g}}$. The effects of fiscal policy on the new options is then precisely determined by the shift $\alpha \cdot g$. Figure 1 represents this idea pictorially. ${ }^{3}$

Our first result delivers a closed-form solution for fiscal multipliers. Using this closed form one can characterize the multiplier quite tightly.

\footnotetext{
${ }^{3}$ The figure is purposefully abstract and meant to convey the notion of a parallel shift only, so we have not labeled either axis and the shape of the sets is purely for illustrative purposes.
} 
liquidity trap

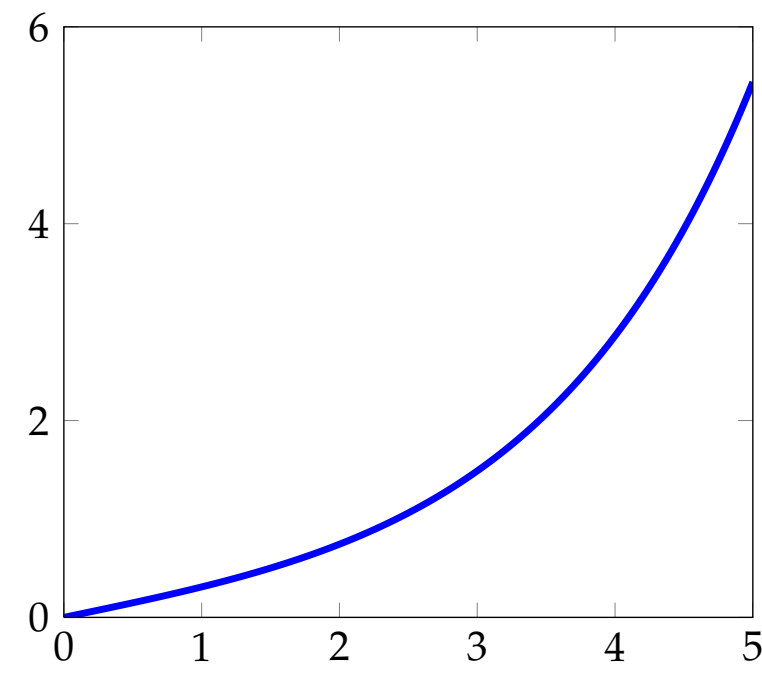

currency union

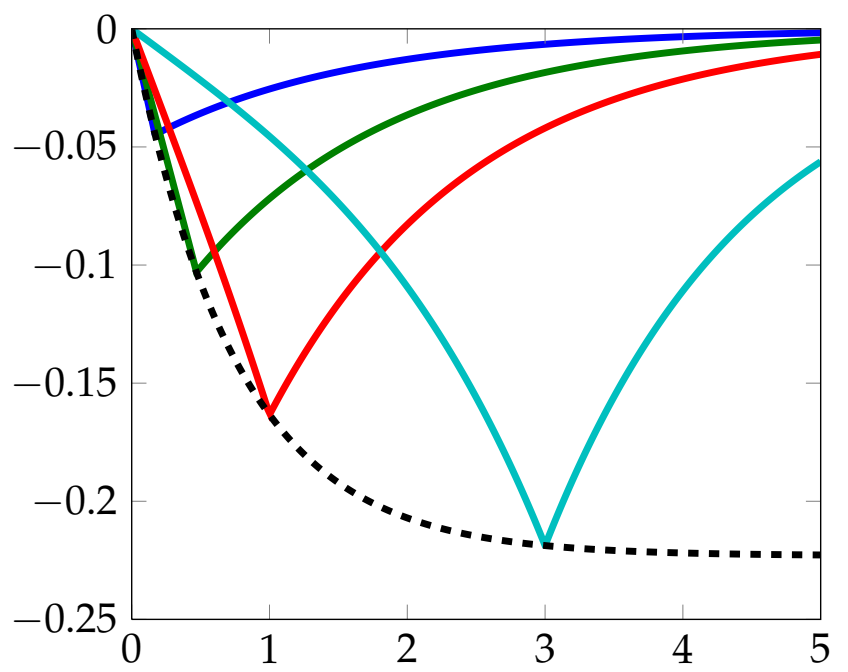

Figure 2: Liquidity trap and currency union consumption multipliers $\alpha_{s}^{c}$ and $\alpha_{s-t}^{c, t, C M}$ as a function of $s$. Each curve for $\alpha_{s-t}^{c, t, C M}$ is plotted for different values of $t \in\{0.25,0.5,1,3\}$. The black dashed line shows the lower envelope. Parameters are $\sigma=1, \eta=\gamma=1, \epsilon=6$, $\phi=3, \lambda=0.14$ and $\alpha=0.4$.

Proposition 1 (Closed Economy Multiplier). The fiscal multipliers are given by

$$
\alpha_{s}^{c}=\hat{\sigma}^{-1} \kappa(1-\xi) e^{-\bar{v} s}\left(\frac{e^{(\bar{v}-v) s}-1}{\bar{v}-v}\right) .
$$

The instantaneous consumption fiscal multiplier is zero $\alpha_{0}^{c}=0$, but is positive, increasing and convex for large s so that $\lim _{s \rightarrow \infty} \alpha_{s}^{c}=\infty$.

The left panel of figure 2 displays these consumption multipliers $\alpha_{s}^{c}$ as a function of $s$ for a standard calibration. The proposition states that current spending has no effect on consumption: $\alpha_{0}^{c}=0$. By implication, changes in spending that are very temporary are expected to have negligible effects on consumption and have an output multiplier that is near unity. As stated earlier, the effects of government spending on consumption work through inflation. Current spending does affect the current inflation rate and this affects the growth rate of consumption. However, since this higher inflation is so short-lived the lower growth rate for consumption has no significant stretch of time to impact the level of consumption.

In contrast, spending that takes place in the far future can have a very large impact. The further out into the future, the larger the impact, since $\alpha_{s}^{c}$ is increasing in $s$. Indeed, in the limit the effect becomes unbounded, since $\lim _{s \rightarrow \infty} \alpha_{s}^{c}=\infty$. The logic behind these 
results is that spending at $s>0$ increases inflation over the entire interval of time $[0, s]$. This then lowers the real interest over this same time interval and lowers the growth rate of consumption. Since the long-run consumption level is fixed, the lower growth rate raises the level of consumption. This rise in consumption in turn leads to higher inflation, creating a feedback cycle. The larger the interval $[0, s]$ over which these effect have time to act, the larger is the effect on consumption.

The fact that fiscal multipliers are unbounded as $s \rightarrow \infty$ stands in strong contrast to the zero multiplier at $s=0$. It also has important implications. For example, a positive path for spending $\left\{g_{t}\right\}$ that is very backloaded can create a very large response for consumption. This is the case if the shock to spending is very persistent.

Example $1\left(\mathrm{AR}(1)\right.$ spending). Suppose $g_{t}=g e^{-\rho_{g} t}$, then if $\rho_{g}>-v>0$ the response of consumption $c_{t}$ is finite and given by

$$
\int \alpha_{s}^{c} g e^{-\rho_{g}(t+s)} d s=\frac{\hat{\sigma}^{-1} \mathcal{K}(1-\xi)}{\left(\rho_{g}+v\right)\left(\rho_{g}+\bar{v}\right)} g e^{-\rho_{g} t}
$$

The condition $\rho_{g}>-v>0$ requires spending to revert to zero fast enough to prevent the integral from being infinite.

Some paths for spending imply an infinite value for $\int_{0}^{\infty} \alpha_{s}^{c} g_{s} d s$. For instance, this is the case in the example above when $\rho_{g}<-v$. How should one interpret such cases? Technically, this may invalidate our approximation. However, we think the correct economic conclusion to draw is that spending will have an explosive positive effect on consumption. One way to see this is to truncate the path of spending $\left\{g_{t}\right\}$, by setting $g_{t}=0$ for all $t \geq T$ for some large $T$. This ensures that $\int_{0}^{T} \alpha_{s}^{c} g_{s} d s$ is finite but the response is guaranteed to be very large if the cutoff is large.

Next, we ask how fiscal multipliers are affected by the degree of price stickiness. Departures from the neoclassical benchmark, where the consumption multiplier is negative, require some stickiness in prices. Perhaps surprisingly, the resulting Keynesian effects turn out to be decreasing in the degree of price stickiness.

Proposition 2 (Price Stickiness). The fiscal multipliers $\left\{\alpha_{s}^{c}\right\}$

1. are zero when prices are rigid $\kappa=0$;

2. are increasing in price flexibility $\kappa$;

3. converge to infinity, $\alpha_{s}^{c} \rightarrow \infty$, in the limit as prices become fully flexible so that $\kappa \rightarrow \infty$. 
The logic for these results relies on the fact that spending acts on consumption through inflation. At one extreme, if prices were perfectly rigid then inflation would be fixed at zero and spending has no effect on consumption. As prices become more flexible spending has a greater impact on inflation and, hence, on consumption. Indeed, in the limit as prices become perfectly flexible, inflation becomes so responsive that the effects on consumption explode.

Recall that our fiscal multipliers are calculated under the assumption that the path for interest rates remains unchanged when spending rises. These results seem less counterintuitive when one realizes that such a monetary policy, insisting on keeping interest rates unchanged, may be deemed to be looser when prices are more flexible and inflation reacts more. Of course, this is precisely the relevant calculation when the economy finds itself in a liquidity trap, so that interest rates are up against the zero lower bound.

\subsection{Summary Fiscal Multipliers}

Up to now we have discussed properties of fiscal multipliers $\left\{\alpha_{s}^{c}\right\}$. Usually, fiscal multipliers are portrayed as a single number that summarizes the impact of some change in spending on output or consumption, perhaps conditional on the state of the economy or monetary policy. This requires collapsing the entire sequence of fiscal multipliers $\left\{\alpha_{s}^{c}\right\}$ into a single number $\bar{\alpha}$, which we shall call a summary fiscal multiplier.

At time $t$ we shock spending from zero to $\left\{g_{t+s}\right\}$ and wish to summarize the impact on current consumption $c_{t}$ by the ratio

$$
\bar{\alpha}=\frac{\int_{0}^{\infty} \alpha_{s}^{c} g_{s} d s}{\int_{0}^{\infty} \alpha_{s}^{g} g_{s} d s}
$$

The numerator is simply the impact on consumption, while the denominator introduces a weighting sequence $\left\{\alpha_{s}^{g}\right\}$ to summarize the path of spending into a single number. One natural candidate is to have the denominator represent a present value by setting $\alpha_{S}^{g}=e^{-\int_{0}^{s} r_{z} d z}, \alpha_{S}^{g}=e^{-\int_{0}^{S}\left(i_{t}-\pi_{t}\right) d z}$ or $\alpha_{S}^{g}=e^{-\rho s}$. It is common in the literature to compute multipliers using current spending only as follows:

$$
\bar{\alpha}=\frac{\int_{0}^{\infty} \alpha_{s}^{c} g_{s} d s}{g_{0}}
$$

One can interpret this as an extreme (Dirac) weighting function $\alpha_{g}$ that puts full mass at $s=0$ and no weight on any $s>0$.

We could also take an average for the impact on consumption $\int_{0}^{\infty} \alpha_{s}^{c} g_{t+s} d s$ at each 
moment in time $t$ in the numerator,

$$
\frac{\int \lambda_{t}^{c} \int_{0}^{\infty} \alpha_{s}^{c} g_{t+s} d s d t}{\int_{0}^{\infty} \alpha_{s}^{g} g_{s} d s}
$$

where $\left\{\lambda_{t}^{c}\right\}$ are weights. The summary multipliers above implicitly put full weight on the consumption response at $t=0$, but we can also consider nonzero weights on future consumption responses. For example, we could set $\left\{\lambda_{t}^{c}\right\}$ so as to compute the response in the present value of consumption.

As this discussion makes clear there are many possibilities for summary multipliers and no universal criteria to select them. Instead, one can adapt the summary multiplier to the application and relevant policy at hand. The characterizations provided in the previous section have implications for any of these measures. Namely,

1. the instantaneous multiplier is zero: if spending $\left\{g_{t}\right\}$ converges to being concentrated at $t=0$ then $\bar{\alpha}=0$;

2. the multiplier is increasing in flexibility, it is zero with rigid prices $\kappa=0$ and goes to infinity in the limit of flexible prices $\kappa \rightarrow \infty$, provided $g_{t}$ is positive for all $t$.

Example 2. Suppose we have an autoregressive spending path $g_{t}=g e^{-\rho_{g} t}$ for $\rho_{g}>0$. The summary multiplier is independent of $g_{0}$ and given by

$$
\bar{\alpha}=\frac{\int_{0}^{\infty} \alpha_{s}^{c} g_{s} d s}{\int_{0}^{\infty} \alpha_{s}^{g} g_{s} d s}=\frac{\frac{1}{\rho_{g}} \int_{0}^{\infty} \alpha_{s}^{c} e^{-\rho_{g} s} d s}{\frac{1}{\rho_{g}} \int_{0}^{\infty} \alpha_{s}^{g} e^{-\rho_{g} s} d s} .
$$

Both the numerator and denominator are weighted averages of $\left\{\alpha_{s}^{c}\right\}$ and $\left\{\alpha_{s}^{g}\right\}$. Higher values of $\rho_{g}$ then shift weight towards the future. Recall that $\alpha^{c}$ is increasing. If $\alpha^{g}$ is decreasing, as is most natural, then the numerator increases and the denominator decreases with $\rho_{g}$. More persistence leads to higher summary multipliers.

The next result is related to this example and shows that delays in spending increase the summary multiplier.

Proposition 3. Consider two paths $\left\{g_{t}^{a}\right\}$ and $\left\{g_{t}^{b}\right\}$ with $\int_{0}^{\infty} \alpha_{s}^{g} g_{s}^{a} d s=\int_{0}^{\infty} \alpha_{s}^{g} g_{s}^{b} d s$. Suppose $\left\{g_{t}^{b}\right\}_{t \geq 0}$ single crosses $\left\{g_{t}^{a}\right\}_{t \geq 0}$ from below (i.e. there exists $t^{\prime}>0$ such that $g_{t}^{a}>g_{t}^{b}$ for $t<t^{\prime}$ and $g_{t}^{a}<g_{t}^{b}$ for $\left.t>t^{\prime}\right)$. Then $\bar{\alpha}^{b}>\bar{\alpha}^{a}$. 


\subsection{Endogenous Spending and Filling the Gap}

The policies for the spending under consideration may be best thought of as endogenous, because they depend on the state of the economy which itself depends on parameters. As we vary parameters this affects both structural fiscal multipliers $\left\{\alpha_{t}^{c}\right\}$ and the path $\left\{g_{t}\right\}$. Both impact summary fiscal multipliers.

Example 3. Christiano, Eichenbaum and Rebelo (2011) compute summary multipliers in a liquidity trap as a function of parameters, using initial spending in the denominator. Their results suggest that parameter values that make the recession worse also lead to larger multipliers. In some cases this follow because the parameters affect the fiscal multipliers $\left\{\alpha_{s}^{c}\right\}$ directly. For example, this is true for the degree of price flexibility $\kappa$. Higher price flexibility makes the recession worse (Werning, 2012) and also leads to higher fiscal multipliers as shown in Proposition 2. However, in other cases their conclusion rely on the indirect effects that these parameters have on the policy experiment $\left\{g_{t}\right\}$ and, thus, on summary multipliers.

Their setup features Poisson uncertainty, but a deterministic version of their calculations is as follows. ${ }^{4}$ Suppose the economy is in a liquidity trap with zero interest rates for $t \leq T$ and will returns to the natural allocation $c_{t}=g_{t}=0$ for $t \geq T$. Consider fiscal policy interventions that increase spending during the trap, $g_{t}=g$ for $t \leq T$ and $g_{t}=0$ for $t>T$. Higher $T$ leads to a deeper recession (see Werning, 2012), but has no effect on the fiscal multipliers $\left\{\alpha_{t}^{c}\right\}$. However, $T$ does have an indirect effect on the summary multiplier

$$
\bar{\alpha}=\frac{\int_{0}^{T} \alpha_{s}^{c} \bar{g} d s}{\bar{g}}=\int_{0}^{T} \alpha_{s}^{c} d s,
$$

which is increasing and convex in $T$.

If $T$ is larger the summary multiplier is larger, but not because spending is any more effective. For instance, it would be wrong conclude that a stimulus plan that increased spending for a fixed amount of time, such as a year or two, would be more powerful when $T$ is large. Rather, when $T$ is increased then spending during the trap has a bigger effect because we are doing more of it by extending the time interval over which it takes place. Indeed, since this extension backloads spending we know from Proposition 1 that it will be particularly effective. Of course, this extension may be warranted precisely because spending affects the allocation at all prior dates.

Example 4. Another perspective is provided when $g_{t}$ is set as a function of current con-

\footnotetext{
${ }^{4}$ Their parameter $p$, which represents the probability of remaining in the trap, has an effect similar to $T$ in our deterministic setting.
} 
sumption:

$$
g_{t}=-\Psi c_{t},
$$

then the Phillips curve becomes

$$
\dot{\pi}_{t}=\rho \pi_{t}-\kappa(1-(1-\xi) \Psi) c_{t},
$$

and the equilibrium is just as if we had a lower $\kappa$ given by $\hat{\kappa}=\kappa(1-(1-\xi) \Psi)$.

Suppose further that $\Psi=(1-\xi)^{-1}$, so that spending fills the gap and $c_{t}+(1-\xi) g_{t}=$ 0 . Inflation is then zero and the outcome for consumption is as if prices were completely rigid $\hat{\kappa}=0$. With this particular policy in place, consider different values for $\kappa$. The spending path $g_{t}$ does not depend on $\kappa$. However, the equilibrium outcome without spending (i.e. $g_{t}=0$ ) is decreasing in $\kappa$ (see Werning, 2012). Thus, fiscal policy affects consumption more the more flexible prices are. This result is consistent with our more general results in Proposition 2 regarding the effects of price flexibility.

\section{An Open Economy Model of a Currency Union}

We now turn to open economy models similar to Farhi and Werning $(2012 a, b)$ which in turn build on Gali and Monacelli $(2005,2008)$.

The model focuses on a continuum of regions or countries that share a common currency. One interpretation is that these regions are states or provinces within a country. Our analysis is then directly relevant to the literature estimating "local" multipliers, exploiting cross-sectional variation in spending behavior across states in the United States to estimate the effects on income and employment. Another interpretation is to member countries within a currency union, such as the European Monetary Union (EMU). Our analysis then sheds light on the debates over fiscal policy, stimulus versus austerity, for periphery countries.

For concreteness, from now on we will refer to these economic units (regions or countries) simply as countries. We focus on the effects around a symmetric steady state after a fiscal policy is realized in every country. A crucial ingredient is how private agents share risk internationally. We consider the two polar case: (i) incomplete markets, where agents can only trade a risk-free bond; and (ii) complete markets with perfect risk sharing. These two market structures have different implications for fiscal multipliers. 


\subsection{Households}

There is a continuum measure one of countries $i \in[0,1]$. We focus attention on a single country, which we call "home" and can be thought of as a particular value $H \in[0,1]$. We will focus on a one time shock, so that all uncertainty is realized at $t=0$. Thus, we can describe the economy after the realization of the shock as a deterministic function of time.

In every country, there is a representative household with preferences represented by the utility function

$$
\int_{0}^{\infty} e^{-\rho t}\left[\frac{C_{t}^{1-\sigma}}{1-\sigma}+\chi \frac{G_{t}^{1-\sigma}}{1-\sigma}-\frac{N_{t}^{1+\phi}}{1+\phi}\right] d t
$$

where $N_{t}$ is labor, and $C_{t}$ is a consumption index defined by

$$
C_{t}=\left[(1-\alpha)^{\frac{1}{\eta}} C_{H, t}^{\frac{\eta-1}{\eta}}+\alpha^{\frac{1}{\eta}} C_{F, t}^{\frac{\eta-1}{\eta}}\right]^{\frac{\eta}{\eta-1}},
$$

where $C_{H, t}$ is an index of consumption of domestic goods given by

$$
C_{H, t}=\left(\int_{0}^{1} C_{H, t}(j)^{\frac{\epsilon-1}{\epsilon}} d j\right)^{\frac{\epsilon}{\epsilon-1}},
$$

where $j \in[0,1]$ denotes an individual good variety. Similarly, $C_{F, t}$ is a consumption index of imported goods given by

$$
C_{F, t}=\left(\int_{0}^{1} C_{i, t}^{\frac{\gamma-1}{\gamma}} d i\right)^{\frac{\gamma}{\gamma-1}}
$$

where $C_{i, t}$ is, in turn, an index of the consumption of varieties of goods imported from country $i$, given by

$$
C_{i, t}=\left(\int_{0}^{1} C_{i, t}(j)^{\frac{\epsilon-1}{\epsilon}} d j\right)^{\frac{\epsilon}{\epsilon-1}} .
$$

Thus, $\epsilon$ is the elasticity between varieties produced within a given country, $\eta$ the elasticity between domestic and foreign goods, and $\gamma$ the elasticity between goods produced in different foreign countries. An important special case obtains when $\sigma=\eta=\gamma=1$. We call this the Cole-Obstfeld case, in reference to Cole and Obstfeld (1991).

The parameter $\alpha$ indexes the degree of home bias, and can be interpreted as a measure of openness. Consider both extremes: as $\alpha \rightarrow 0$ the share of foreign goods vanishes; as $\alpha \rightarrow 1$ the share of home goods vanishes. Since the country is infinitesimal, the latter 
captures a very open economy without home bias; the former a closed economy barely trading with the outside world.

Households seek to maximize their utility subject to the budget constraints

$$
\dot{D}_{t}=i_{t} D_{t}-\int_{0}^{1} P_{H, t}(j) C_{H, t}(j) d j-\int_{0}^{1} \int_{0}^{1} P_{i, t}(j) C_{i, t}(j) d j d i+W_{t} N_{t}+\Pi_{t}+T_{t}
$$

for $t \geq 0$. In this equation, $P_{H, t}(j)$ is the price of domestic variety $j, P_{i, t}$ is the price of variety $j$ imported from country $i, W_{t}$ is the nominal wage, $\Pi_{t}$ represents nominal profits and $T_{t}$ is a nominal lump-sum transfer. All these variables are expressed in the common currency. The bond holdings of home agents is denoted by $D_{t}$ and the common nominal interest rate within the union is denoted by $i_{t}$.

We sometimes allow for transfers across countries that are contingent on shocks. These transfers may be due to private arrangements in complete financial markets. or due to government arrangements. These transfers can accrue to the government or directly to the agents. This is irrelevant since lump-sum taxes are available. For example, we sometimes consider the assumption of complete markets where agents in different countries can perfectly share risks in a complete set of financial markets. Agents form international portfolios, the returns of which result in international transfers that are contingent on the realization of the shock. A different example is in Section 6 where we consider government spending in the home country paid for by a transfer from the rest of the world. In this case, we have in mind a direct transfer to the government of the home country, or simply spending paid for and made by the rest of the world.

\subsection{Government}

Government consumption $G_{t}$ is an aggregate of different varieties. Importantly, we assume that government spending is concentrated exclusively on domestic varieties

$$
G_{t}=\left(\int_{0}^{1} G_{t}(j)^{\frac{\epsilon-1}{\epsilon}} d j\right)^{\frac{\epsilon}{\epsilon-1}}
$$

For any level of expenditure $\int_{0}^{1} P_{H, t}(j) G_{t}(j) d j$, the government splits its expenditure across varieties to maximize government consumption $G_{t}$. Spending is financed by lump-sum taxes. The timing of these taxes is irrelevant since Ricardian equivalence holds in our basic model. We only examine a potentially non-Ricardian setting in Section 7 where we introduce hand-to-mouth consumers into the model. 


\subsection{Firms}

Technology. A typical firm in the home economy produces a differentiated good using a linear technology

$$
Y_{t}(j)=A_{H, t} N_{t}(j),
$$

where $A_{H, t}$ is productivity in the home country. We denote productivity in country $i$ by $A_{i, t}$.

We allow for a constant employment tax $1+\tau^{L}$, so that real marginal cost deflated by Home PPI is $\frac{1+\tau^{L}}{A_{H, t}} \frac{W_{t}}{P_{H, t}}$. We take this employment tax to be constant and set to offset the monopoly distortion so that $\tau^{L}=-\frac{1}{\varepsilon}$, as is standard in the literature. However, none of our results hinge on this particular value.

Price-setting assumptions. We assume that the Law of One Price holds so that at all times, the price of a given variety in different countries is identical once expressed in the same currency.

We adopt the Calvo price setting framework, where in every period, a randomly flow $\rho_{\delta}$ of firms can reset their prices. Those firms that get to reset their price choose a reset price $P_{t}^{r}$ to solve

$$
\max _{P_{t}^{r}} \int_{0}^{\infty} e^{-\rho_{\delta} s-\int_{0}^{s} i_{t+z} d z}\left(P_{t}^{r} Y_{t+s \mid t}-\left(1+\tau^{L}\right) W_{t} \frac{Y_{t+s \mid t}}{A_{H, t}}\right)
$$

where $Y_{t+k \mid t}=\left(\frac{P_{t}^{r}}{P_{H, t+k}}\right)^{-\epsilon} Y_{t+k}$, taking the sequences for $W_{t}, Y_{t}$ and $P_{H, t}$ as given.

\subsection{Terms of Trade and Real Exchange Rate}

It is useful to define the following price indices: the home Consumer Price Index (CPI) is

$$
P_{t}=\left[(1-\alpha) P_{H, t}^{1-\eta}+\alpha P_{t}^{* 1-\eta}\right]^{\frac{1}{1-\eta}}
$$

the home Producer Price Index (PPI)

$$
P_{H, t}=\left[\int_{0}^{1} P_{H, t}(j)^{1-\epsilon} d j\right]^{\frac{1}{1-\epsilon}},
$$

and $P_{t}^{*}$ is the price index for imported goods. The terms of trade are defined by

$$
S_{t}=\frac{P_{t}^{*}}{P_{H, t}} .
$$


Similarly let the real exchange rate be

$$
\mathcal{Q}_{t}=\frac{P_{t}^{*}}{P_{t}}
$$

\subsection{Equilibrium Conditions}

We now summarize the equilibrium conditions. For simplicity of exposition, we focus on the case where all foreign countries are identical. Because agents face the same sequence of interest rates optimal consumption satisfies

$$
C_{t}=\Theta C_{t}^{*} \mathcal{Q}_{t}^{\frac{1}{\sigma}}
$$

where $\Theta$ is a relative Pareto weight which might depend on the realization of the shocks, the goods market clearing condition

$$
Y_{t}=\left[(1-\alpha) C_{t}+G_{t}\right]\left(\frac{\mathcal{Q}_{t}}{S_{t}}\right)^{-\eta}+\alpha S_{t}^{\gamma} C_{t}^{*} .
$$

We also have the labor market clearing condition

$$
N_{t}=\frac{Y_{t}}{A_{H, t}} \Delta_{t}
$$

where $\Delta_{t}$ is an index of price dispersion $\Delta_{t}=\int_{0}^{1}\left(\frac{P_{H, t}(j)}{P_{H, t}}\right)^{-\epsilon}$ and the Euler equation

$$
\sigma \frac{\dot{C}_{t}}{C_{t}}=i_{t}-\pi_{t}-\rho
$$

where $\pi_{t}=\dot{P}_{t} / P_{t}$ is CPI inflation. Finally, we must include the country-wide budget constraint

$$
N \dot{F} A_{t}=\left(P_{H, t} Y_{t}-P_{t} C_{t}\right)+i_{t} N F A_{t}
$$

where $N F A_{t}$ is the country's net foreign assets at $t$, which for convenience, we measure in home numeraire. We impose a standard no-Ponzi condition, $e^{-\int_{0}^{t} i_{s} d s} N F A_{t} \rightarrow 0$ as $t \rightarrow \infty$.

Absent transfers or insurance across countries we set $N F A_{0}$ to be constant for all shock realizations, and normalize its value to zero. Instead, when markets are complete we require that $\Theta$ does not vary with the shock realization. We then solve for the initial value of $N F A_{0}$ that is needed, for each shock realization. This value can be interpreted as an insurance transfer from the rest of the world. 
Finally with Calvo price setting we have the equations summarizing the first-order condition for optimal price setting. We omit these conditions since we will only analyze a log-linearized version of the model.

\section{National and Local Fiscal Multipliers in Currency Unions}

To compute local multipliers we study the log-linearized equilibrium conditions around a symmetric steady state with zero inflation. We denote the deviations of output and public consumption on home goods relative to steady state output by

$$
\begin{gathered}
c_{t}=(1-\mathcal{G})\left(\log \left(Y_{t}-G_{t}\right)-\log (Y-G)\right) \approx \frac{Y_{t}-G_{t}-(Y-G)}{Y} \\
y_{t}=\log \left(Y_{t}\right)-\log (Y) \approx \frac{Y_{t}-Y}{Y} \quad g_{t}=\mathcal{G}\left(\log G_{t}-\log G\right) \approx \frac{G_{t}-G}{Y}
\end{gathered}
$$

where $\mathcal{G}=\frac{G}{Y}$ denotes the steady state share of government spending in output. Then we have, up to a first order approximation,

$$
y_{t}=c_{t}+g_{t}
$$

Note that $c_{t}$ does not represent private domestic total consumption (of home and foreign goods); instead it is private consumption (domestic and foreign) of domestic goods. In a closed economy the two coincide, but in an open economy, for our purposes, the latter is more relevant and convenient.

The log linearized system can then be written as a set of differential equations

$$
\begin{aligned}
\dot{\pi}_{H, t} & =\rho \pi_{H, t}-\kappa\left(c_{t}+(1-\xi) g_{t}\right)-\lambda \hat{\sigma} \alpha(\omega-1) c_{t}^{*}-(1-\mathcal{G}) \lambda \hat{\sigma} \alpha \omega \theta, \\
\dot{c}_{t} & =\hat{\sigma}^{-1}\left(i_{t}^{*}-\pi_{H, t}-\rho\right)-\alpha(\omega-1) \dot{c}_{t}^{*},
\end{aligned}
$$

with an initial condition and the definition of the variable $\theta$,

$$
\begin{aligned}
c_{0} & =(1-\mathcal{G})(1-\alpha) \theta+c_{0}^{*}, \\
\theta & =\frac{\rho}{\alpha} \frac{1}{1-\mathcal{G}} \frac{1}{\Gamma} \mathrm{nfa}_{0}+\Omega \rho \int_{0}^{\infty} e^{-\rho t}\left(c_{t}-c_{t}^{*}\right) d t
\end{aligned}
$$

and either

$$
\mathrm{nfa}_{0}=0
$$


if markets are incomplete or

$$
\theta=0
$$

if markets are complete, where $\mathrm{nfa}_{0}=\frac{N F A_{0}}{Y}$ is the normalized deviation of the initial net foreign asset position from ( $\mathrm{nfa}_{0}=0$ at the symmetric steady state) and $\theta=\log \Theta$ is the wedge in the log-linearized Backus-Smith equation $(\theta=0$ at the symmetric steady state). In these equations, we have used the following definitions: $\lambda=\rho_{\delta}\left(\rho+\rho_{\delta}\right), \kappa=\lambda(\hat{\sigma}+\phi)$, $\xi=\frac{\hat{\sigma}}{\hat{\sigma}+\phi}$ and

$$
\begin{aligned}
\omega & =\sigma \gamma+(1-\alpha)(\sigma \eta-1), \\
\hat{\sigma} & =\frac{\sigma}{1-\alpha+\alpha \omega} \frac{1}{1-\mathcal{G}^{\prime}} \\
\Gamma & =1+(1-\mathcal{G})\left(\frac{\omega}{\sigma}-1\right) \hat{\sigma}(1-\alpha), \\
\Omega & =\frac{\left(\frac{\omega}{\sigma}-1\right) \hat{\sigma}}{\Gamma} .
\end{aligned}
$$

Equation (4) is the New-Keynesian Philips Curve. Equation (5) is the Euler equation. Equation (6) is derived from the requirement that the terms of trade are predetermined at $t=0$ because prices are sticky and the exchange rate is fixed. Finally equation (7) together with either (8) or (9) depending on whether markets are incomplete or complete, represents the country budget constraint. In the Cole-Obstfeld case $\sigma=\eta=\gamma=1$, we have $\Omega=0$ so that the complete and incomplete markets solutions coincide. Away from the Cole-Obstfeld case, the complete and incomplete markets solutions differ. The incomplete markets solution imposes that the country budget constraint (7) with nfa $\mathrm{a}_{0}=0$, while the complete markets solution solves for the endogenous value of $\mathrm{nfa}_{0}$ that ensures that the country budget constraint (7) holds with $\theta=0$. This can be interpreted as an insurance payment from the rest of the world.

These equations form a linear differential system with forcing variables $\left\{g_{t}, g_{t}^{*}, i_{t}^{*}\right\}$. It will prove useful to define the following two numbers $v$ and $\bar{v}$ (the eigenvalues of the system):

$$
v=\frac{\rho-\sqrt{\rho^{2}+4 \kappa \hat{\sigma}^{-1}}}{2} \quad \bar{v}=\frac{\rho+\sqrt{\rho^{2}+4 \kappa \hat{\sigma}^{-1}}}{2} .
$$

\subsection{Home Government Spending}

We first consider the experiment where the only shock is home government spending, so that $i_{t}^{*}=\rho, g_{t}^{*}=y_{t}^{*}=c_{t}^{*}=0$. Note that if $g_{t}=0$ throughout then $\theta=0$ and $y_{t}=c_{t}=0$. We shall compute the deviations from this steady state when $g_{t} \neq 0$. 
The assumptions one makes about financial markets can affect the results. We consider, in turn, both the cases of complete markets and incomplete markets.

Complete Markets. We start by studying the case where markets are complete. This assumption is representative of most of the literature. The assumption is often adopted as a benchmark due to its tractability. The key implication is that consumption is insured against spending shocks. In equilibrium, private agents make arrangements with the rest of the world to receive transfers when spending shoots up and, conversely, to make transfers when spending shoots down. As a result, government sending shocks to not affect consumption on impact. Formally, we have $\theta=0$, so the system becomes

$$
\begin{aligned}
\dot{\pi}_{H, t} & =\rho \pi_{H, t}-\kappa\left(c_{t}+(1-\xi) g_{t}\right), \\
\dot{c}_{t} & =-\hat{\sigma}^{-1} \pi_{H, t},
\end{aligned}
$$

with initial condition

$$
c_{0}=0 .
$$

Because the system is linear, we can write

$$
\begin{aligned}
c_{t} & =\int_{-t}^{\infty} \alpha_{s}^{c, t, C M} g_{t+s} d s, \\
\pi_{H, t} & =\int_{-t}^{\infty} \alpha_{s}^{\pi, t, C M} g_{t+s} d s,
\end{aligned}
$$

where the superscript $C M$ stands for complete markets. Note two important differences with the closed economy case. First, there are both forward-and backward-looking effects from government spending; the lower bound in these integrals is now given by $-t$ instead of 0 . At every point in time consumption is pinned down by the terms of trade which depend on past inflation. Second, the multipliers depend on calendar time $t$.

Proposition 4 (Complete Markets). Suppose that markets are complete, then the fiscal multipliers are given by

$$
\alpha_{s}^{c, t, C M}= \begin{cases}-\hat{\sigma}^{-1} \mathcal{K}(1-\xi) e^{-v s} \frac{1-e^{(v-\bar{v})(s+t)}}{\bar{v}-\nu} & s<0 \\ -\hat{\sigma}^{-1} \mathcal{K}(1-\xi) e^{-\bar{\nu} s} \frac{1-e^{-(\bar{\nu}-v) t}}{\bar{v}-v} & s \geq 0 .\end{cases}
$$

It follows that

1. for $t=0$ we have $\alpha_{s}^{c, t, C M}=0$ for all $s$, 
2. for $t>0$ we have $\alpha_{s}^{c, t, C M}<0$ for all $s$,

3. for $t \rightarrow \infty$ we have $\alpha_{s-t}^{c, t, C M} \rightarrow 0$ for all $s$,

4. spending at zero and infinity have no impact: $\alpha_{-t}^{c, t, C M}=\lim _{s \rightarrow \infty} \alpha_{s}^{c, t, C M}=0$.

The right panel of figure 2 displays consumption multipliers for a standard calibration. Consumption multipliers are very different in an open economy with a fixed exchange rate. For starters, part (1) says that the initial response of consumption is always zero, simply restating the initial condition above that $c_{0}=0$. This follows from the fact that the terms of trade are predetermined and complete markets insure consumption.

Part (2) proves that the consumption response at any other date is actually negative. Note that the Euler equation and the initial condition together imply that

$$
c_{t}=-\hat{\sigma}^{-1} \log \frac{P_{H, t}}{P_{H}}
$$

Government spending increases demand, leading to inflation, a rise in $P_{H, t}$. In other words, it leads to an appreciation in the terms of trade and this loss in competitiveness depresses private demand, from both domestic and foreign consumers. Although we have derived this result in a specific setting, we expect it to be robust. The key ingredients are that consumption depends negatively on the terms of trade and that government spending creates inflation.

It may seem surprising that the output multiplier is necessarily less than one whenever the exchange rate is fixed, because this contrasts sharply with our conclusions in a closed economy with a fixed interest rate. They key here is that a fixed exchange rate implies a fixed interest rate, but the reverse is not true. We expand on this idea in the next subsection.

Part (3) says that the impact of government spending at any date on private consumption vanishes in the long run. This exact long run neutrality relies on the assumption of complete markets; otherwise, there are potential long-run neoclassical wealth effects from accumulation of foreign assets.

Part (4) says that spending near zero and spending in the very far future have negligible impacts on consumption at any date. Spending near zero affects inflation for a trivial amount of time and thus have has insignificant effects on the level of home prices. Similarly, spending in the far future has vanishing effects on inflation at any date.

Example 5 (Complete Markets, $\mathrm{AR}(1))$. Suppose that $g_{t}=g e^{-\rho_{g} t}$ and that markets are 
complete. Then

$$
c_{t}=-g e^{\nu t} \frac{1-e^{-\left(v+\rho_{g}\right) t}}{v+\rho_{g}} \frac{\hat{\sigma}^{-1} \kappa(1-\xi)}{\bar{v}+\rho_{g}} .
$$

For $g>0$, this example shows that $c_{t}$ is always negative. In other words, in the open economy model with complete markets, output always expands less than the increase in government spending. The intuition is simple. Because the terms of trade are predetermined, private spending on home goods is also predetermined so that $c_{0}=0$. Government spending initially leads to inflation because the total (public and private) demand for home goods is increased in the short run. With fixed nominal interest rates, inflation depresses real interest rates, leading to a decreasing path of private consumption of domestic goods, so that $c_{t}$ becomes negative. The inflationary pressures are greatest at $t=0$ and they then recede over time as public and private demand decrease. Indeed at some point in time, inflation becomes negative and in the long run, the terms of trade return to their steady state value. At that point, private consumption of domestic goods $\hat{c}_{t}$ reaches its minimum and starts increasing, returning to 0 in the long run. The crucial role of inflation in generating $c_{t}<0$ is most powerfully illustrated in the rigid price case. When prices are entirely rigid, we have $\kappa=0$ so that $c_{t}=0$ throughout. $^{5}$

An interesting observation is that the openness parameter $\alpha$ enters Proposition 4 or Example 5 only through its effect on $\hat{\sigma} .^{6}$ As a result, in the Cole-Obstfeld case $\sigma=\eta=\gamma=$ 1 and the private consumption multipliers $\alpha_{s}^{c, t}$ are completely independent of openness $\alpha$. Away from the Cole-Obstfeld case, $\alpha_{s}^{c, t}$ depends on $\alpha$, but its dependence can be positive or negative depending on the parameters. ${ }^{7}$

Next, we ask how fiscal multipliers are affected by the degree of price stickiness.

Proposition 5 (Price Stickiness). The fiscal multipliers $\left\{\alpha_{s}^{c, t, C M}\right\}$ depend on price flexibility as follows:

1. when prices are rigid so that $\kappa=0$, we have $\alpha_{s}^{c, t, C M}=0$ for all s and $t$;

2. when prices become perfectly flexible $\kappa \rightarrow \infty$, then for all $t$, the function $s \rightarrow \alpha_{s}^{c, t, C M}$ converges in distributions to $-(1-\xi)$ times a Dirac distribution concentrated at $s=0$, implying that $\int_{-t}^{\infty} \alpha_{s}^{c, t, C M} g_{t+s} d s=-(1-\xi) g_{t}$ for all (continuous and bounded) paths of government spending $\left\{g_{t}\right\}$.

\footnotetext{
${ }^{5}$ Note that the above calculation is valid even if $\rho_{g}<0$, as long as $\bar{v}+\rho_{g}>0$. If this condition is violated, then $c_{t}$ is $-\infty$ for $g>0$ and $+\infty$ for $g<0$.

${ }^{6}$ Recall that $\hat{\sigma}=\frac{\sigma}{1+\alpha[(\sigma \gamma-1)+(\sigma \eta-1)-\alpha(\sigma \eta-1)]} \frac{1}{1-\mathcal{G}}$.

${ }^{7}$ For example, when $\sigma \eta>1$ and $\sigma \gamma>1, \alpha_{s}^{c, t}$ is increasing in $\alpha$ for $\alpha \in\left[0, \min \left\{\frac{(\sigma \gamma-1)+(\sigma \eta-1)}{2(\sigma \eta-1)}, 1\right\}\right]$ and decreasing in $\alpha$ for $\alpha \in\left[\min \left\{\frac{(\sigma \gamma-1)+(\sigma \eta-1)}{2(\sigma \eta-1)}, 1\right\}, 1\right]$.
} 
Unlike in the liquidity trap, fiscal multipliers do not explode when prices become more flexible. In a liquidity trap, government spending sets into motion a feedback loop between consumption and inflation: government spending increases inflation, which lower real interest rates, increases private consumption, further increasing inflation etc. ad infinitum. This feedback loop is non-existent in a currency union: government spending increases inflation, appreciates the terms of trade, reduces private consumption, reducing the inflationary pressure. Instead, the allocation converges to the flexible price allocation $c_{t}=-(1-\xi) g_{t}$ when prices become very flexible. At the flexible price allocation, private consumption is entirely determined by contemporaneous government spending. Hence the function $\alpha_{s}^{c, t, C M}$ of $s$ converges in distributions to $-(1-\xi)$ times a Dirac function at $s=0$. This implies that fact that for $s=0, \lim _{\kappa \rightarrow \infty} \alpha_{s}^{c, t, C M}=-\infty$ and for $s \neq 0$, $\lim _{\kappa \rightarrow \infty} \alpha_{s}^{c, t, C M}=0$.

One can reinterpret the neoclassical outcome with flexible prices as applying to the case with rigid prices and a flexible exchange rate that is adjusted to replicate the flexible price allocation. The output multiplier is then less than one. The first result says that with rigid prices but fixed exchange rates, output multipliers are equal to one. In this sense, the comparison between fixed with flexible exchange rates confirms the conventional view from the Mundell-Flemming model that fiscal policy is more effective with fixed exchange rates (see e.g. Dornbusch, 1980). This is consistent with the simulation findings in Corsetti, Kuester and Muller (2011).

Incomplete Markets. We now turn our attention to the case where markets are incomplete. Although the complete market assumption is often adopted for tractability, we believe incomplete markets may be a better approximation to reality in most cases of interest.

A shock to spending may create income effects that affect consumption and labor responses. The complete markets solution secures transfers from the rest of the world that effectively cancel these income effects. As a result, the incomplete markets solution is in general different from the complete market case. One exception is the Cole-Obstfeld case, where $\sigma=\eta=\gamma=1$.

With incomplete markets, the system becomes

$$
\begin{aligned}
\dot{\pi}_{H, t} & =\rho \pi_{H, t}-\kappa\left(c_{t}+(1-\xi) g_{t}\right)-(1-\mathcal{G}) \lambda \hat{\sigma} \alpha \omega \theta, \\
\dot{c}_{t} & =-\hat{\sigma}^{-1} \pi_{H, t},
\end{aligned}
$$


with initial condition

$$
\begin{aligned}
c_{0} & =(1-\mathcal{G})(1-\alpha) \theta, \\
\theta & =\Omega \rho \int_{0}^{\infty} e^{-\rho t} c_{t} d t .
\end{aligned}
$$

We denote the consumption multipliers with a superscript $I M$, which stands for incomplete markets. We denote by $\hat{t}$ the time such that

$$
\frac{e^{\nu \hat{t}}}{1-e^{v \hat{t}}}=\omega \frac{\hat{\sigma}}{\hat{\sigma}+\phi} \frac{\alpha}{1-\alpha}
$$

We also define

$$
\bar{\Omega}=\frac{\Omega(1-\xi)}{1-\Omega(1-\mathcal{G})(1-\alpha)\left[\frac{\rho}{\bar{v}}+\frac{v}{\bar{v}} \omega \frac{\hat{\sigma}}{\hat{\sigma}+\phi} \frac{\alpha}{1-\alpha}\right]} .
$$

Note that $\bar{\Omega}=0$ in the Cole-Obstfeld case.

Proposition 6 (Incomplete Markets). Suppose that markets are incomplete, then

$$
\alpha_{s}^{c, t, I M}=\alpha_{s}^{c, t, C M}+\delta_{s}^{c, t, I M}
$$

where $\alpha_{s}^{c, t, C M}$ is the complete markets consumption multiplier characterized in Proposition 4 and

$$
\delta_{s}^{c, t, I M}=(1-\mathcal{G}) \alpha \rho \bar{\Omega}\left[e^{\nu t} \frac{1-\alpha}{\alpha}-\left(1-e^{\nu t}\right) \omega \frac{\hat{\sigma}}{\hat{\sigma}+\phi}\right] e^{-\rho(s+t)}\left(1-e^{v(s+t)}\right) .
$$

The difference $\delta_{s}^{c, t, I M}$ is 0 in the Cole-Obstfeld case $\sigma=\eta=\gamma=1$. Away from the ColeObstfled case, the sign of $\delta_{s}^{c, t, I M}$ is the same as the sign of $\left(\frac{\omega}{\sigma}-1\right)(t-\hat{t})$; moreover, $\delta_{-t}^{c, t, I M}=0$ and $\lim _{s \rightarrow \infty} \delta_{s}^{c, t, I M}=0$.

The difference between the complete and incomplete market solution vanishes in the Cole-Obstfeld case. Although, away from the this case $\delta_{s}^{c, t, I M}$ is generally nonzero, it necessarily changes signs (both as a function of $s$ for a given $t$, and as a function of $t$, for a given $s$ ). In this sense, incomplete markets cannot robustly overturn the conclusion of Proposition 4 and guarantee positive multipliers for consumption.

With complete markets

$$
\theta=0,
$$


while with incomplete markets

$$
\theta=-\rho \bar{\Omega} \int_{0}^{\infty} g_{s} e^{-\rho s}\left(1-e^{\nu s}\right) d s
$$

This means that with complete markets, home receives an endogenous transfer $\mathrm{nfa}_{0}$ from the rest of the world following a government spending shock. In the Cole-Obstfeld case, this transfer is zero, but away from this case, this transfer is nonzero. The difference between these two solutions can then be obtained as the effect of this endogenous transfer, using the analysis in Section 6.2.

Example 6 (Incomplete Markets, $\mathrm{AR}(1)$ ). Suppose that $g_{t}=g e^{-\rho_{g} t}$. Then

$$
c_{t}=(1-\mathcal{G}) \alpha \theta\left[e^{\nu t} \frac{1-\alpha}{\alpha}-\left(1-e^{\nu t}\right) \omega \frac{\hat{\sigma}}{\hat{\sigma}+\phi}\right]-g e^{\nu t} \frac{1-e^{-\left(\nu+\rho_{g}\right) t}}{v+\rho_{g}} \frac{\hat{\sigma}^{-1} \mathcal{K}(1-\xi)}{\bar{v}+\rho_{g}},
$$

where

$$
\theta=-g \rho \bar{\Omega} \frac{-v}{\left(\rho+\rho_{g}\right)\left(\bar{v}+\rho_{g}\right)} .
$$

The second term of the right hand side of the expression for $c_{t}$ is identical to the complete markets solution identified in Example 5. When $g>0$, it is always negative. The first term on the right hand side of this expression arises only because markets are incomplete. Indeed in vanishes in the Cole-Obstfeld case where the complete and incomplete markets solution coincide. It is small compared to the first term in the neighborhood of the ColeObstfeld case. It necessarily changes sign over time. For $\frac{\omega}{\sigma}$ close to $1, \theta$ is of the same sign as $1-\frac{\omega}{\sigma}$. Hence the first term is of the same sign as $1-\frac{\omega}{\sigma}$ for small $t$ and of the opposite sign for large $t$.

\subsection{Understanding Closed versus Open Economy Multipliers}

Figure 2 provides a sharp illustration of the difference between a liquidity trap and a currency union. In a liquidity trap, consumption multipliers are positive, increase with the date of spending, and becomes arbitrarily large for long-dated spending. By contrast, in a currency union, consumption multipliers are negative, V-shaped and bounded as a function of the date of spending, and asymptote to zero for long-dated spending.

Before continuing it is useful to pause to develop a deeper understanding of the key difference between the closed and open economy results. The two models are somewhat different-the open economy features trade in goods and the closed economy does notyet they are quite comparable. Indeed, we will highlight that the crucial difference lies 
in monetary policy, not model primitives. Although a fixed exchange rate implies a fixed nominal interest rate, the converse is not true.

To make the closed economy and open economies more comparable we consider the limit of the latter as $\alpha \rightarrow 0$. This limit represents a closed economy in the sense that preferences display an extreme home bias and trade is zero. To simplify, we focus on the case of complete markets so that $\theta=0$. Even in this limit case, the closed and open economy multipliers differ. This might seems surprising since, after all, both experiments consider the effects of government spending for a fixed nominal interest rate. To understand the difference, we allow for an initial devaluation.

Consider then the open economy model in the closed-economy limit $\alpha \rightarrow 0$ and let $e_{0}$ denote the new value for the exchange rate after the shock in log deviations relative to its steady-state value (so that $e_{0}=0$ represents no devaluation). The only difference introduced in the system by such one-time devaluation is a change the initial condition to ${ }^{8}$

$$
c_{0}=\hat{\sigma}^{-1} e_{0} .
$$

The exchange rate devaluation $e_{0}$ depreciates the initial terms of trade one for one and increases the demand for home goods through an expenditure switching effect. Of course, this stimulative effect is present in the short run, but vanishes in the long run once prices have adjusted.

Now if in the closed economy limit of the open economy model, we set the devaluation $e_{0}$ so that $\hat{\sigma}^{-1} e_{0}$ exactly equals the initial consumption response $\int_{0}^{\infty} \alpha_{s}^{c} g_{t+s} d s$ of the closed economy model, i.e.

$$
e_{0}=\int_{0}^{\infty} \kappa(1-\xi) e^{-\bar{v} s}\left(\frac{e^{(\bar{v}-v) s}-1}{\bar{v}-v}\right) g_{s} d s,
$$

then we find exactly the same response for consumption and inflation as in the closed economy model. This means that if we combined the government spending shock with

\footnotetext{
${ }^{8}$ The full system allowing for a flexible exchange rate and an independent monetary policy $i_{t}$ is (with $\theta=0$ and $c_{t}^{*}=0$ )

$$
\begin{aligned}
\dot{\pi}_{H, t} & =\rho \pi_{H, t}-\kappa\left(c_{t}+(1-\xi) g_{t}\right), \\
\dot{c}_{t} & =\hat{\sigma}^{-1}\left(i_{t}-\pi_{H, t}-\rho\right), \\
\dot{e}_{t} & =i_{t}-i_{t}^{*}
\end{aligned}
$$
}

with initial condition

$$
c_{0}=\hat{\sigma}^{-1} e_{0} .
$$

If we set $i_{t}=i_{t}^{*}$ then $\dot{e}_{t}=0$ so that $e_{t}=e_{0}$, which amounts to a one-time devaluation. 
an initial devaluation given by (10), then the multipliers of the closed economy limit of the open economy model would coincide with those of the closed economy model. ${ }^{9}$

This analysis shows that the policy analysis conducted for our closed economy model implicitly combines a shock to government spending with a devaluation. ${ }^{10}$ In contrast, our open economy analysis assumes fixed exchange rates, ruling out such devaluations. The positive response of consumption in the closed economy model relies entirely on this one-time devaluation. Thus, the key difference between the two models is in monetary policy, not whether the economy is modeled as open or closed. Indeed, we have taken the closed-economy limit $\alpha \rightarrow 0$, but the results hold more generally: the degree of openness $\alpha$ matters only indirectly through its impact on $\hat{\sigma}, v$ and $\bar{v}$ and in the Cole-Obstfeld case, $\alpha$ actually does not even affect these parameters.

\subsection{Domestic Government Spending on Foreign Goods}

We now analyze the impact of domestic government spending on foreign goods $g_{F, t}$. We treat both the cases of complete and incomplete markets.

When markets are complete, spending on foreign goods has absolutely no effect, $c_{t}=$ 0 and $\pi_{H, t}=0$. This follows because this shock to spending is then effectively fully paid for by foreigners and it does not affect the demand for home goods. Of course, if we consider intermediate cases, where the home government spends on both home and foreign goods, the conclusion is that only the spending on home goods has any effect. Part of government spending leaks abroad, increasing the demand for foreign goods. Only the increase in home goods provides stimulus to the home economy.

When markets are incomplete these negative conclusions are reinforced. The effect of a spending shock in the home country that is spent entirely on foreign goods is identical to the response in a complete market setting together with a negative transfer equal to the present value of the spending shock,

$$
\mathrm{nfa}_{0}=-\int_{0}^{\infty} e^{-\rho t} g_{F, t} d t
$$

\footnotetext{
${ }^{9}$ Note that the size of this devaluation is endogenous and grows without bound as prices become more flexible i.e. as $\kappa$ increases. This explains why large multipliers are possible with high values of $\kappa$ in the closed economy model: they are associated with large devaluations.

${ }^{10}$ To see what this implies, suppose the spending shock has a finite life so that $g_{t}=0$ for $t \geq T$ for some $T$ and that monetary policy targets inflation for $t \geq T$. In the closed economy model inflation is always positive and the price level does not return to its previous level. In contrast, in the open economy model with a fixed exchange rate (no devaluation) inflation is initially positive but eventually negative and the price level returns to its initial steady state value. Indeed, if $g_{t}>0$ for $t<T$ and $g_{t}=0$ for $t \geq T$ for some $T$, then inflation is strictly negative for $t \geq T$ and the price level falls towards its long run value asymptotically.
} 
In the short run, it reduces the domestic demand for home goods, depressing output. In the long run, output is increased by the standard neoclassical wealth effects on labor supply. We refer the reader to Section 6.2 below where we provide a detailed analysis of the effects of such transfers.

\subsection{Foreign Government Spending}

We now consider the effects of a shock to spending by a foreign country. In particular, suppose the rest of the world changes its government spending adopting the path $\left\{g_{t}^{*}\right\}$ but that spending remains unchanged in the home country so that $g_{t}=0$ for all $t \geq 0$. We must take a stand on monetary policy within the union. We assume that monetary policy adjusts so as to implement the flexible price allocation for the union as a whole. This amounts to

$$
c_{t}^{*}=-(1-\xi) g_{t}^{*} \quad \pi_{t}^{*}=0 \quad i_{t}^{*}-\rho=-\hat{\sigma}(1-\xi) \dot{g}_{t}^{*},
$$

so that at the union level we obtain the neoclassical effect from spending and no inflation. The interest rate is adjusted depending on the growth rate of spending; if the shock is transitory, so that $g_{0}^{*}>0$ and $\dot{g}_{t}^{*}<0$, the interest rate $i_{t}^{*}$ rises.

To see how this affects the home country we assume, for simplicity, the case of complete markets so that $\theta=0$. The system is then

$$
\begin{aligned}
\dot{\pi}_{H, t} & =\rho \pi_{H, t}-\kappa c_{t}+\lambda \hat{\sigma} \alpha(\omega-1)(1-\xi) g_{t}^{*}, \\
\dot{c}_{t} & =-\hat{\sigma}^{-1} \pi_{H, t}-(1-\alpha(\omega-1))(1-\xi) \dot{g}_{t}^{*},
\end{aligned}
$$

with the initial condition

$$
c_{0}=-(1-\xi) g_{0}^{*}
$$

Proposition 7 (Foreign Government Spending). Suppose that markets are complete, then

$$
c_{t}=-e^{\nu t} \alpha(\omega-1)(1-\xi) g_{0}^{*}-(1-\alpha(\omega-1))(1-\xi) g_{t}^{*}+\int_{-t}^{\infty} \alpha_{s}^{c, t, C M *} g_{s} d s,
$$

where

$$
\alpha_{s}^{c, t, C M *}= \begin{cases}\hat{\sigma}^{-1} \mathcal{K}(1-\xi)\left[1+\left(\frac{\hat{\sigma}}{\hat{\sigma}+\phi}-1\right) \alpha(\omega-1)\right] e^{-v s \frac{1-e^{(v-\bar{v})(s+t)}}{\bar{v}-v}} & s<0, \\ \hat{\sigma}^{-1} \mathcal{K}(1-\xi)\left[1+\left(\frac{\hat{\sigma}}{\hat{\sigma}+\phi}-1\right) \alpha(\omega-1)\right] e^{-\bar{v} s \frac{1-e^{(v-\bar{v}) t}}{\bar{v}-v}} & s \geq 0 .\end{cases}
$$

There are now both direct effects and indirect effects. The indirect effects work through 
inflation, which affect the terms of trade and, hence, demand for home goods. To simplify the expressions consider the Cole-Obstfeld case where $\omega=1$. To isolate the direct effects set $\kappa=0$, so that there is no inflation and $\alpha_{s}^{c, t, C M *}=0$. The demand for home goods is then equal to $c_{t}=-(1-\xi) g_{t}^{*}$. When foreign spending rises it depresses private spending by foreigners, lowering the demand for home output. When $\kappa>0$, the indirect effect works through inflation and is positive in the Cole-Obstfeld case. The lower demand for home goods creates deflation at home, which makes the economy more competitive. The lower prices then increase the demand for home goods. ${ }^{11}$

Example 7 (Foreign Government Spending, AR(1)). Suppose that $g_{t}^{*}=g^{*} e^{-\rho_{g} t}$ and that markets are complete, then we have

$$
c_{t}=-e^{\nu t}(1-\xi) g^{*}\left[1-\frac{1-e^{-\left(v+\rho_{g}\right) t}}{\rho_{g}+v} \frac{\rho_{g}\left(\rho+\rho_{g}\right)+\left[\lambda-\rho_{g}\left(\rho+\rho_{g}\right)\right] \alpha(\omega-1)}{\rho_{g}+\bar{v}}\right] .
$$

In the Cole-Obstfeld case, so that $\omega=1$, this implies that $c_{0}$ is negative if $g^{*}$ is positive. If $\rho_{g}+v<0$ then $c_{t}$ will remain negative. If instead $\rho_{g}+v>0$ then $c_{t}$ starts out negative, but eventually switches signs.

This results suggests that a temporary increase in government spending abroad accompanied by monetary tightening to ensure no inflation abroad induces a recession at home. This fits a common narrative regarding the post German reunification in the early 90s. The fiscal expansion was combined with a monetary contraction in Germany, so as to avoid inflation. The quasi-fixed exchange rate arrangements of the EMS forced other countries to follow suit and tighten monetary policy, negatively affecting their economic performance.

\section{Spending Paid by Foreigners and Transfer Multipliers}

\subsection{Home Government Spending Paid by Foreign}

In this section, we examine what happens when the home country "doesn't pay" for the increase in government spending at home. We show that this modification can make an important difference and produce larger multipliers. This consideration is likely to be important in practice: indeed, a large part of the "local multiplier" literature considers

\footnotetext{
${ }^{11}$ As the expressions show, the result that $\alpha_{s}^{c, t, C M *} \geq 0$ holds in the neighborhood of the Cole-Obstfeld case or whenever $\omega<1$. Sufficiently away from the Cole-Obstfeld case in the direction of $\omega>1$ one may obtain $\alpha_{s}^{c, t, C M *}<0$.
} 
experiments where government spending is not paid by the economic unit under consideration.

The only difference with Section 5.1 is that we now have

$$
\theta=\frac{\rho}{\alpha} \frac{1}{1-\mathcal{G}} \frac{1}{\Gamma} \mathrm{nfa}_{0}+\Omega \rho \int_{0}^{\infty} e^{-\rho t} c_{t} d t
$$

where

$$
\mathrm{nfa}_{0}=\int_{0}^{\infty} e^{-\rho t} g_{t} d t
$$

is the transfer from foreign to home that pays for the increase in government spending. We provide a closed form solution in the appendix, but the expressions for this closed form solution are quite involved. Here we focus on two cases that are more tractable and amenable to clean intuitions: the Cole-Obstfeld case and the case of entirely rigid prices.

In in the Cole-Obstfeld case $\sigma=\eta=\gamma=1$, we have $\Gamma=1$ and $\Omega=0$. We denote the consumption multipliers with a superscript $P F$, which stands for paid for.

Proposition 8 (Spending Paid by Foreign, Cole-Obstfeld). Suppose that government spending is paid for by foreign, then in the Cole-Obstfeld case $\sigma=\eta=\gamma=1$, we have

$$
\alpha_{s}^{c, t, P F}=\alpha_{s}^{c, t, C M}+\delta_{s}^{c, t, P F},
$$

where $\alpha_{s}^{c, t, C M}$ is the complete markets consumption multiplier characterized in Proposition 4 and

$$
\delta_{s}^{c, t, P F}=\left[e^{v t} \frac{1-\alpha}{\alpha}-\left(1-e^{v t}\right) \frac{1}{1-\mathcal{G}} \frac{1}{\frac{1}{1-\mathcal{G}}+\phi}\right] \rho e^{-\rho(s+t)} .
$$

The sign of $\delta_{s}^{c, t, I M}$ is the same as that of $(\hat{t}-t)$; moreover, $\delta_{-t}^{c, t, I M}=\rho \frac{1-\alpha}{\alpha}$ and $\lim _{s \rightarrow \infty} \delta_{s}^{c, t, I M}=0$.

When government spending is paid for by foreign, the associated transfer from foreign to home increases the demand for home goods as long as there is home bias. It also creates a wealth effect which tends to reduces the output of home goods. In the short run, output is demand-determined leading to a Keynesian expansion effect on private consumption. The neoclassical effect only kicks in in the long run when the terms of trade have appreciated significantly. The Keynesian effect dominates dominates in the short run and the neoclassical wealth effect dominates in the long run.

Example 8 (Spending Paid by Foreign, Cole-Obstfeld, AR(1)). Suppose that $g_{t}=g e^{-\rho_{g} t}$. 
In the Cole-Obstfeld case $\sigma=\eta=\gamma=1$, we have

$$
\begin{aligned}
& c_{t}=g\left[e^{\nu t} \frac{1-\alpha}{\alpha}-\left(1-e^{\nu t}\right) \frac{1}{1-\mathcal{G}} \frac{1}{\frac{1}{1-\mathcal{G}}+\phi}\right] \frac{\rho}{\rho+\rho_{g}} \\
& -g e^{v t}\left(\frac{1-e^{-\left(v+\rho_{g}\right) t}}{v+\rho_{g}}\right) \kappa(1-\xi) \frac{1-\mathcal{G}}{\bar{v}+\rho_{g}} .
\end{aligned}
$$

Moreover we have $c_{0}=g \frac{1-\alpha}{\alpha} \frac{\rho}{\rho+\rho_{g}}$ and $\lim _{t \rightarrow \infty} c_{t}=-g \frac{1}{1-\mathcal{G}} \frac{1}{\frac{1}{1-\mathcal{G}}+\phi} \frac{\rho}{\rho+\rho_{g}}$.

Note that the second term on the right hand side of the expression for $c_{t}$ in Proposition 8 is simply the term identified in Proposition 4 in the complete markets case. The first term is different from the corresponding term in Proposition 6 in the incomplete markets case, which is simply 0 in the Cole-Obstfeld case. The discrepancy arises precisely because government spending is now paid for by foreign. This takes the form of a transfer from foreign to home.

It is particularly useful to look at the predictions of this proposition for $t=0$ and $t \rightarrow \infty$. In the case of a stimulus $g>0$, we have $c_{0}>0>\lim _{t \rightarrow \infty} c_{t}$. Following a positive stimulus shock, we can get $c_{0}>0$ and actually $c_{t}>0$ for some time (because $\theta>0$ ) and eventually $\hat{c}_{t}<0$. The conclusion would be that an unpaid for fiscal stimulus at home has a larger consumption multiplier in the short run and smaller in the long run. This is true as long as there is home bias $\alpha<1$. The reason is that the associated transfer redistributes wealth from foreign to home consumers. This increases the demand for home goods because of home bias. In the neoclassical model with flexible prices, there would be an appreciation of the terms of trade and a reduction in the output of home goods because of a neoclassical wealth effect. With sticky prices, prices cannot adjust in the short term, and so this appreciation cannot take place right away, and so the output of home goods increases. In the long run, prices adjust and we get the neoclassical effect.

We now turn to the case of entirely rigid prices.

Proposition 9 (Spending Paid by Foreign, Rigid Prices). Suppose that government spending is paid for by foreigners and that prices are entirely rigid, then

$$
\alpha_{s}^{c, t, P F}=\frac{1}{1-\Omega(1-\mathcal{G})(1-\alpha)} \frac{1}{\Gamma} \frac{1-\alpha}{\alpha} \rho e^{-\rho(t+s)} .
$$

The solution takes a particularly simple form in the case where government spending follows an AR(1).

Example 9 (Spending Paid by Foreign, Rigid Prices, AR(1)). Suppose that $g_{t}=g e^{-\rho_{g} t}$, 
and that prices are entirely rigid, then

$$
c_{t}=g \frac{1}{1-\Omega(1-\mathcal{G})(1-\alpha)} \frac{1}{\Gamma} \frac{\rho}{\rho+\rho_{g}} \frac{1-\alpha}{\alpha} .
$$

With rigid prices, when government spending is not paid by foreign, we have $c_{t}=0$. Instead when government spending is paid by foreign, we have $c_{t}>0$ when $g>0$. The annuity value of the transfer received from foreign is simple $g \frac{\rho}{\rho+\rho_{g}}$. The multiplier effect of this transfer on output is given by $\frac{1-\alpha}{\alpha} \frac{1}{1-\Omega(1-\mathcal{G})(1-\alpha)} \frac{1}{\Gamma}$. In the Cole-Obstfeld case, this multiplier takes the simple form $\frac{1-\alpha}{\alpha}$, the relative expenditure share of home goods. Note that this effect is permanent despite the fact that the government spending shock is meanreverting. This is because the effect of the transfer is permanent. We provide a detailed intuition for this result in Section 6.2 below.

The lesson of this section is that we can partly overturn the conclusion of Proposition 4 when government spending is paid for by foreign. The reason is that the associated transfer from foreign stimulates private consumption and output.

\subsection{Transfer Multipliers}

The previous section highlights the role of transfers. We do it in the Cole-Obstfeld case where $\sigma=\eta=\gamma=1$ for simplicity. The next proposition characterizes the response of the economy to a marginal increase in transfers nfa $_{0}$. Because the model is linear, we can represent this dependence by some coefficients $\beta^{c, t}$ such that

$$
\begin{aligned}
\hat{c_{t}} & =\beta^{c, t} \mathrm{nfa}_{0}, \\
\pi_{H, t} & =\beta^{\pi, t} \mathrm{nfa}_{0} .
\end{aligned}
$$

Proposition 10 (Transfer Multipliers, Cole-Obstfeld). In the Cole-Obstfeld case $\sigma=\eta=$ $\gamma=1$, transfer multipliers are given by

$$
\beta^{c, t}=e^{v t} \rho \frac{1-\alpha}{\alpha}-\left(1-e^{v t}\right) \rho \frac{1}{\frac{1}{1-\mathcal{G}}+\phi} .
$$

We can also compute the effects of transfers on inflation $\beta^{\pi, t}=-v e^{v t}\left[\rho \frac{1-\alpha}{\alpha}+\rho \frac{1}{\frac{1}{1-\mathcal{G}}+\phi}\right]$ and on the terms of trade $\beta^{s, t}=-\left[1-e^{\nu t}\right]\left[\rho \frac{1-\alpha}{\alpha}+\rho \frac{1}{\frac{1}{1-\mathcal{G}}+\phi}\right]$ (note that the terms of trade gap equals accumulated inflation $s_{t}=-\int_{0}^{t} \pi_{H, s} d s$ ). The presence of the discount factor $\rho$ in all these expressions is natural because what matters is the annuity value $\rho \mathrm{nfa}_{0}$ of the 
transfer.

Transfers have opposite effects on output in the short and long run. In the short run, when prices are rigid, there is a Keynesian effect due to the fact that transfers stimulate the demand for home goods: $\beta^{c, 0}=\rho \frac{1-\alpha}{\alpha}$. In the long run, when prices adjust, the neoclassical wealth effect on labor supply lowers output: $\lim _{t \rightarrow \infty} \beta^{c, t}=-\rho \frac{1}{\frac{1}{1-\mathcal{G}}+\phi}$. In the medium run, the speed of adjustment, from the Keynesian short-run response to the neoclassical longrun response, is controlled by the degree of price flexibility $\kappa$, which affects $v .{ }^{12}$

Note that the determinants of the Keynesian and neoclassical wealth effects are very different. The strength of the Keynesian effect hinges on the relative expenditure share of home goods $\frac{1-\alpha}{\alpha}$ : the more closed the economy, the larger the Keynesian effect. The strength of the neoclassical wealth effect depends on the elasticity of labor supply $\phi$ : the more elastic labor supply, the larger the neoclassical wealth effect.

Positive transfers also increase home inflation. The long-run cumulated response in the price of home produced goods equals $\rho \frac{1-\alpha}{\alpha}+\rho \frac{1}{\frac{1}{1-\mathcal{G}}+\phi}$. The first term $\rho \frac{1-\alpha}{\alpha}$ comes from the fact that transfers increase the demand for home goods, due to home bias. The second term $\rho \frac{1}{\frac{1}{1-\mathcal{G}}+\phi}$ is due to a neoclassical wealth effect that reduces labor supply, raising the wage. How fast this increase in the price of home goods occurs depends positively on the flexibility of prices through its effect on $v{ }^{13}$

These effects echo the celebrated Transfer Problem controversy of Keynes (1929) and Ohlin (1929). With home bias, a transfer generates a boom when prices are sticky, and a real appreciation of the terms of trade when prices are flexible. The neoclassical wealth effect associated with a transfer comes into play when prices are flexible, and generates an output contraction and a further real appreciation.

The analysis generalizes away from the Cole-Obstfeld case, but the corresponding formulas become more involved. However, a simple formula arises in the case where prices are rigid, in which cases transfer multipliers are constant.

Proposition 11 (Transfer Multipliers, Rigid Prices). When prices are entirely rigid, the transfer multipliers are given by

$$
\beta^{c, t}=\frac{1}{1-\Omega(1-\mathcal{G})(1-\alpha)} \frac{1}{\Gamma} \frac{1-\alpha}{\alpha} \rho .
$$

We can also treat the closed economy limit $(\alpha=0)$ and the fully open economy limit $(\alpha=1)$.

\footnotetext{
${ }^{12}$ Note that $v$ is decreasing in $\kappa$, with $v=0$ when prices are rigid $(\kappa=0)$, and $v=-\infty$ when prices are flexible $(\kappa=\infty)$.

${ }^{13}$ Recall that $v$ is decreasing in the degree of price flexibility $\kappa$.
} 
Proposition 12 (Transfer Multipliers). In the closed economy limit we have $\lim _{\alpha \rightarrow 0} \beta^{c, t}=\infty$. In the fully open economy limit we have $\lim _{\alpha \rightarrow 0} \beta^{c, t}=0$.

The intuition for this proposition is that the Keynesian effect of transfers is commensurate with the relative expenditure share on home goods $\frac{1-\alpha}{\alpha}$. This proposition underscores that transfers are much more stimulative than government spending, the more so, the more closed the economy. This robust negative dependence of transfer multipliers $\beta^{c, t}$ on openness $\alpha$ should be contrasted with the lack of clear dependence on openness of government spending multipliers $\alpha_{s}^{c, t, C M}$ noted above (indeed in the Cole Obstfeld case, $\alpha_{s}^{c, t, C M}$ is independent of $\alpha$ ).

\section{Liquidity Constraints and Non-Ricardian Effects}

In this section, we explore non-Ricardian effects of fiscal policy in a closed and open economy setting. To do so, we follow Campbell and Mankiw (1989), Mankiw (2000) and Gali et al. (2007) and introduce hand-to-mouth consumers, a tractable way of modeling liquidity constraints. The latter paper studied the effects of government spending under a Taylor rule in a closed economy. Instead, our focus here is on liquidity traps and currency unions.

\subsection{Hand to Mouth in the Closed Economy}

The model is modified as follows. A fraction $1-\chi$ of agents are optimizers, and a fraction $\chi$ are hand-to-mouth. Optimizers are exactly as before. Hand-to-mouth agents cannot save or borrow, and instead simply consume their labor income in every period, net of lump-sum taxes $t_{t}^{r}$. These lump-sum taxes are allowed to differ between optimizers $\left(t_{t}^{o}\right)$ and hand-to-mouth agents $\left(t_{t}^{r}\right)$.

We log-linearize around a steady state where optimizers and hand-to-mouth consumers have the same consumption and supply the same labor. In the appendix, we show that the model can be summarized by the following two equations

$$
\begin{aligned}
\dot{c}_{t} & =\tilde{\sigma}^{-1}\left(i_{t}-\bar{r}_{t}-\pi_{t}\right)+\tilde{\Theta}_{n} \dot{g}_{t}-\tilde{\Theta}_{\tau} \dot{t}_{t}^{r}, \\
\dot{\pi}_{t} & =\rho \pi_{t}-\kappa\left[c_{t}+(1-\xi) g_{t}\right],
\end{aligned}
$$

where $\tilde{\sigma}, \tilde{\Theta}_{n}$ and $\tilde{\Theta}_{\tau}$ are positive constants defined in the appendix. The presence of hand-to-mouth consumers introduces two new terms in the Euler equation, one involving government spending and the other one involving taxes-both direct determinants of the 
consumption of hand-to-mouth agents. These terms drop out without hand-to-mouth consumers, since $\chi=0$ implies $\tilde{\Theta}_{n}=\tilde{\Theta}_{\tau}=0$ and $\tilde{\sigma}=\hat{\sigma}$.

As before we define

$$
\tilde{v}=\frac{\rho-\sqrt{\rho^{2}+4 \kappa \tilde{\sigma}^{-1}}}{2} \quad \quad \bar{v}=\frac{\rho+\sqrt{\rho^{2}+4 \kappa \tilde{\sigma}^{-1}}}{2} .
$$

We denote the corresponding multipliers with a HM superscript, which stands for handto-mouth.

Proposition 13 (Closed Economy Multiplier, Hand-to-Mouth). With hand to mouth consumers, we have

$$
c_{t}=\tilde{\Theta}_{n} g_{t}-\tilde{\Theta}_{\tau} t_{t}^{r}+\int_{0}^{\infty} \alpha_{s}^{c, H M} g_{t+s} d s-\int_{0}^{\infty} \gamma_{s}^{c, H M} t_{t+s}^{r} d s,
$$

where

$$
\begin{aligned}
& \alpha_{s}^{c, H M}=\left(1+\frac{\tilde{\Theta}_{n}}{1-\xi}\right) \tilde{\alpha}_{s}^{c, H M} \quad \gamma_{s}^{c, H M}=\frac{\tilde{\Theta}_{\tau}}{1-\tilde{\xi}} \tilde{\alpha}_{s}^{c, H M} . \\
& \tilde{\alpha}_{s}^{c, H M}=\tilde{\sigma}^{-1} \kappa(1-\xi) e^{-\tilde{\tilde{v} s}}\left(\frac{e^{(\tilde{v}-\tilde{v}) s}-1}{\tilde{v}-\tilde{v}}\right) .
\end{aligned}
$$

With hand-to-mouth consumers, we have additional terms in the closed form solution. We start with the tax terms. Lower taxes on hand-to-mouth consumers today increases consumption. This is intuitive since this redistributes income either from future hand-tomouth consumers or optimizing agents towards current hand-to-mouth consumers, i.e. from consumers with a low propensity to consume today out of current income to consumers with a high propensity to consume today out of current income. Lower taxes on hand-to-mouth consumers in the future stimulates their consumption tomorrow, which increases inflation today and in the future, reducing the real interest rate and increasing consumption of optimizing agents today.

We continue with the government spending terms. Higher government spending today increases consumption. This is because government spending increases the income of current hand to mouth consumers, and hence their consumption. Higher government spending in the future increases consumption more than in the model with only optimizing agents. The reason is that government spending in the future partly falls on handto-mouth consumers, stimulating their consumption tomorrow, which increases inflation today and in the future, reducing the real interest rate and increasing consumption of optimizing agents today. 
Hence both for taxes and government spending, hand-to-mouth consumers introduces additional traditional Keynesian effects (through the income of current hand-tomouth consumers), and additional New-Keynesian effects (through the income of future hand-to-mouth consumers feeding back into inflation). The New Keynesian effects become more potent when prices are more flexible (when $\kappa$ increases), which is intuitive since these effects work through inflation. This is not the case for the Keynesian effects.

It is important to understand how these results depend on fixed interest rates, due, say, to a binding zero lower bound. Away from this bound, monetary policy could be chosen to replicate the flexible price allocation with zero inflation. The required nominal interest rate is impacted by the presence of hand-to-mouth consumers

$$
i_{t}=\tilde{\sigma}\left[(1-\xi)+\tilde{\Theta}_{n}\right] \dot{g}_{t}+\tilde{\sigma} \tilde{\Theta}_{\tau} \dot{t}_{t}^{r},
$$

but consumption is not

$$
c_{t}=-(1-\xi) g_{t} .
$$

Hence away from the zero bound, we get the neoclassical multiplier, which is determined completely statically and does not depend on the presence of hand-to-mouth consumers. ${ }^{14}$ In contrast, whenever monetary policy does not or cannot replicate the flexible price allocation, then hand-to-mouth consumers do make a difference for fiscal multipliers. Gali et al. (2007) consider a Taylor rule which falls short of replicating the flexible price allocation. Here we have focused on fixed interest rates, motivated by liquidity traps.

Panel (a) in Figure 3 displays the response of consumption over time for an AR(1) government spending shock. The shock mean reverts quite quickly here. The response of consumption is non-monotonic in the fraction of hand-to-mouth agents. Higher $\chi$ implies there are more hand to mouth consumers and fewer savers. At the same time, future spending hand-to-mouth agents also contributes to inflation, and hence to current spending by optimizing agents. Which of these effects dominates is ambiguous. Overall, for our simulations, more hand-to-mouth agents means higher multipliers at impact, but the effect reverses at some point in time. This figure makes clear that the interplay between the increase in future spending by hand-to-mouth agents, the inflation that it generates, and the current and future spending decisions of optimizing agents is extremely potent and can generate very large multipliers.

\footnotetext{
${ }^{14}$ Note however that hand-to-mouth agents might change the associated allocation of optimizers. They just don't matter for the aggregate allocation.
} 


\subsection{Hand to Mouth in the Open Economy}

We now turn to the open economy version with hand to mouth agents. In the appendix, we show that the system becomes

$$
\begin{aligned}
\dot{\pi}_{H, t} & =\rho \pi_{H, t}-\tilde{\kappa}\left(c_{t}+(1-\xi) g_{t}\right)-(1-\mathcal{G}) \lambda \tilde{\sigma} \tilde{\alpha} \tilde{\omega} \theta, \\
\dot{c}_{t} & =-\tilde{\sigma}^{-1} \pi_{H, t}+\tilde{\Theta}_{n \dot{g}_{t}}-\tilde{\Theta}_{\tau} \dot{t}_{t}^{r},
\end{aligned}
$$

with initial condition

$$
c_{0}=\tilde{\Theta}_{n} g_{0}-\tilde{\Theta}_{\tau} t_{0}^{r},
$$

for some constants $\tilde{\kappa}, \tilde{\alpha}, \tilde{\omega}, \tilde{\sigma}, \tilde{\Theta}_{n}$ and $\tilde{\Theta}_{\tau}$ defined in the appendix. As usual, we define

$$
\tilde{v}=\frac{\rho-\sqrt{\rho^{2}+4 \kappa \tilde{\sigma}^{-1}}}{2} \quad \quad \bar{v}=\frac{\rho+\sqrt{\rho^{2}+4 \kappa \tilde{\sigma}^{-1}}}{2} .
$$

Proposition 14 (Complete Markets). With hand-to-mouth agents and complete markets for optimizers, we have

$$
c_{t}=\tilde{\Theta}_{n} g_{t}-\tilde{\Theta}_{\tau} \tau_{t}^{r}+\int_{-t}^{\infty} \alpha_{s}^{c, t, H M} g_{t+s} d s-\int_{-t}^{\infty} \gamma_{s}^{c, t, H M} t_{t+s}^{r} d s,
$$

where

$$
\begin{aligned}
& \alpha_{s}^{c, t, H M}=\left(1+\frac{\tilde{\Theta}_{n}}{1-\xi}\right) \tilde{\alpha}_{s}^{c, t} \quad \gamma_{s}^{c, t, H M}=\frac{\tilde{\Theta}_{\tau}}{1-\xi} \tilde{\alpha}_{s}^{c, t} \\
& \tilde{\alpha}_{s}^{c, t}= \begin{cases}-\tilde{\sigma}^{-1} \mathcal{K}(1-\xi) e^{-\tilde{\nu} s} \frac{1-e^{(\tilde{v}-\tilde{v})(s+t)}}{\tilde{v}-\tilde{v}} & s<0 \\
-\tilde{\sigma}^{-1} \mathcal{K}(1-\xi) e^{-\tilde{\tilde{\nu}} s} \frac{1-e^{-(\tilde{v}-\tilde{v}) t}}{\tilde{v}-\tilde{v}} & s \geq 0\end{cases}
\end{aligned}
$$

Just as in the closed economy case, hand-to-mouth consumers introduce additional Keynesian effects (through the spending of current hand-to-mouth agents) and New Keynesian effects through cumulated inflation (via past and future hand-to-mouth agents). Just as in the closed economy case, the Keynesian effects increase consumption in response to contemporaneous positive government spending shocks. The difference with the closed economy case is that the New Keynesian effects tend to depress consumption in response to positive government spending shocks. A pure illustration of the Keynesian effect is initial consumption $c_{0}$ (for which New Keynesian effects are 0 ), which is not 0 anymore, but instead $c_{0}=\tilde{\Theta}_{n} g_{0}-\tilde{\Theta}_{\tau} t_{0}^{r}$.

The right panel of Figure 3 displays consumption over time for an AR(1) government 
liquidity trap

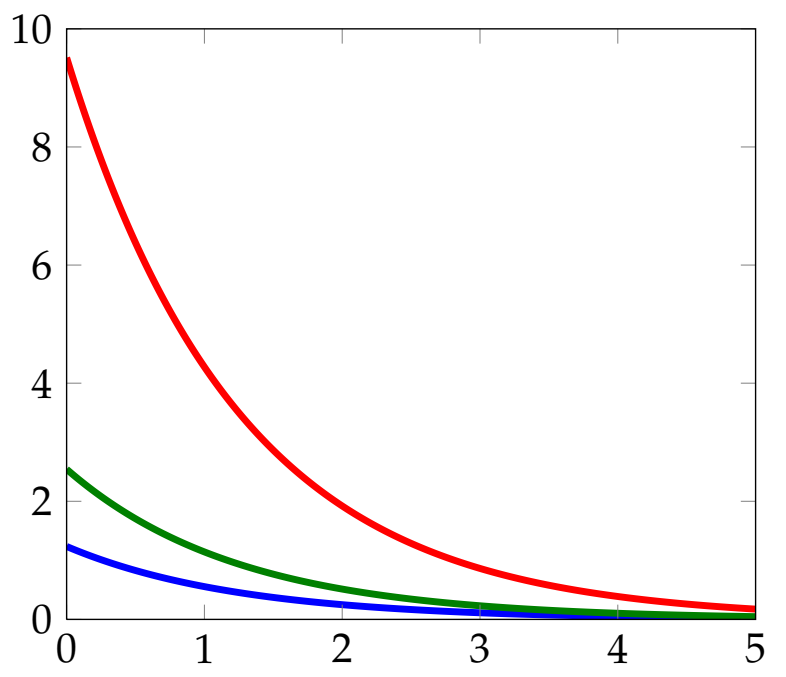

currency union

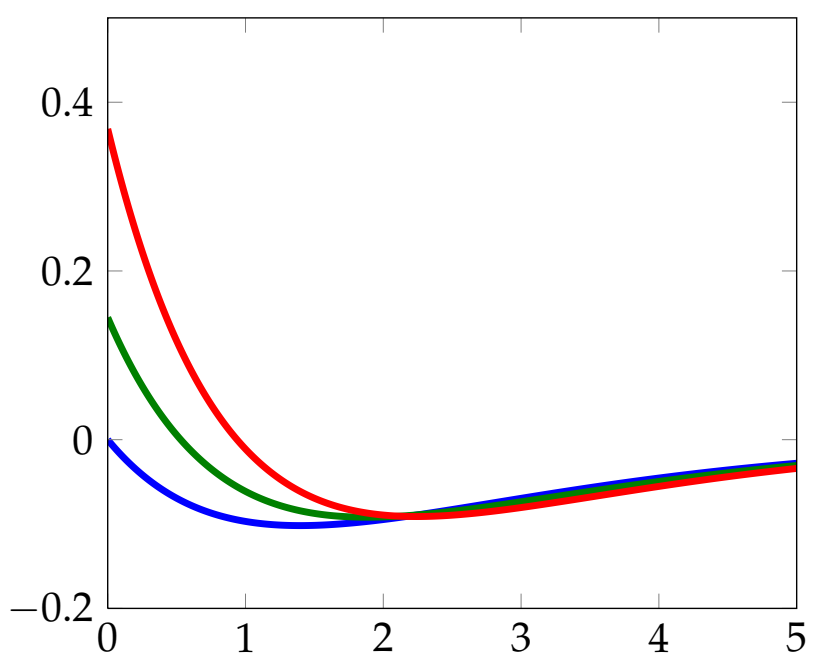

Figure 3: Consumption response to an AR1 government spending shock with mean reversion $\rho_{g}=0.8$ (with a half life of $\frac{\log 2}{\rho_{g}} \approx .87$ ), for with different fractions $\chi$ of handto-mouth agents: $\chi=0$ (blue), $\chi=0.075$ (green) and $\chi=0.15$ (red). Panel (a) shows liquidity trap case while panel (b) shows the currency union case. Parameters are $\sigma=1$, $\eta=\gamma=1, \epsilon=6, \phi=3, \lambda=0.14$ and $\alpha=0.4$.

spending shock. The higher the fraction $\chi$ of hand to mouth agents, the higher the initial response of consumption. At impact, more hand-to-mouth agents means more Keynesian effects through the spending of hand to mouth agents but less new New-Keynesian effects through the spending of optimizing agents. More hand-to-mouth agents unambiguously raises aggregate spending at impact. The effect reverses at some point in time because of the higher terms of trade appreciation when there are more hand-to-mouth agents (and current and future government spending is small).

\section{References}

Acconcia, Antonio, Giancarlo Corsetti, and Saverio Simonelli, "Mafia and Public Spending: Evidence on the Fiscal Multiplier from a Quasi-experiment," CEPR Discussion Papers, C.E.P.R. Discussion Papers April 2011.

Auerbach, Alan and Yuriy Gorodnichenko, "Fiscal Multipliers in Recession and Expansion," in "Fiscal Policy after the Financial Crisis" NBER Chapters, National Bureau of Economic Research, Inc, 2012. 
Barro, Robert J. and Charles J. Redlick, "Macroeconomic Effects from Government Purchases and Taxes," NBER Working Papers 15369, National Bureau of Economic Research, Inc September 2009.

Campbell, John Y. and N. Gregory Mankiw, "Consumption, Income and Interest Rates: Reinterpreting the Time Series Evidence," in "NBER Macroeconomics Annual 1989, Volume 4" NBER Chapters, National Bureau of Economic Research, Inc, 1989, pp. 185246.

Chodorow-Reich, G., L. Feiveson, Z. Liscow, and W.G. Woolston, "Does State Fiscal Relief During Recessions Increase Employment? Evidence from the American Recovery and Reinvestment Act," University of California at Berkeley Working Paper 2011.

Christiano, Lawrence, Martin Eichenbaum, and Sergio Rebelo, "When Is the Government Spending Multiplier Large?," Journal of Political Economy, 2011, 119 (1), 78-121.

Clemens, J. and S. Miran, "The Effects of State Budget Cuts on Employment and Income," Harvard University Working Paper 2010.

Cohen, Lauren, Joshua D. Coval, and Christopher Malloy, "Do Powerful Politicians Cause Corporate Downsizing?," NBER Working Papers, National Bureau of Economic Research, Inc March 2010.

Cole, Harold L. and Maurice Obstfeld, "Commodity Trade and International Risk Sharing: How Much Do Financial Markets Matter?," Journal of Monetary Economics, 1991, 28 (1), 3-24.

Corsetti, Giancarlo, Keith Kuester, and Gernot J. Muller, "Floats, Pegs and the Transmission of Fiscal Policy," Journal Economia Chilena (The Chilean Economy), August 2011, $14(2), 5-38$.

Dornbusch, Rudiger, "Exchange Rate Economics: Where Do We Stand?," Brookings Papers on Economic Activity, 1980, 11 (1, Tenth), 143-206.

Eggertsson, Gauti B., "What Fiscal Policy is Effective at Zero Interest Rates?," in "NBER Macroeconomics Annual 2010, Volume 25" NBER Chapters, National Bureau of Economic Research, Inc, 2011, pp. 59-112.

Erceg, Christopher J. and Jesper Linde, "Fiscal Consolidation in an Open Economy," American Economic Review, May 2012, 102 (3), 186-91. 
Farhi, Emmanuel and Ivan Werning, "Dealing with the Trilemma: Optimal Capital Controls with Fixed Exchange Rates," NBER Working Papers 18199, National Bureau of Economic Research, Inc June 2012.

_ and _ , "Fiscal Unions," NBER Working Papers 18280, National Bureau of Economic Research, Inc June 2012.

Fishback, Price V. and Valentina Kachanovskaya, "In Search of the Multiplier for Federal Spending in the States During the Great Depression," NBER Working Papers, National Bureau of Economic Research, Inc November 2010.

Gali, Jordi and Tommaso Monacelli, "Monetary Policy and Exchange Rate Volatility in a Small Open Economy," Review of Economic Studies, 07 2005, 72 (3), 707-734.

_ and _ , "Optimal monetary and fiscal policy in a currency union," Journal of International Economics, September 2008, 76 (1), 116-132.

_ , J. David Lopez-Salido, and Javier Valles, "Understanding the Effects of Government Spending on Consumption," Journal of the European Economic Association, 03 2007, 5 (1), 227-270.

Gordon, Robert J. and Robert Krenn, "The End of the Great Depression 1939-41: Policy Contributions and Fiscal Multipliers," NBER Working Papers 16380, National Bureau of Economic Research, Inc September 2010.

Keynes, J.M., “The German transfer problem," The Economic Journal, 1929, 39 (153), 1-7.

Mankiw, Gregory N., "The Savers-Spenders Theory of Fiscal Policy," American Economic Review, May 2000, 90 (2), 120-125.

Nakamura, Emi and Jon Steinsson, "Fiscal Stimulus in a Monetary Union: Evidence from U.S. Regions," NBER Working Papers 17391, National Bureau of Economic Research, Inc September 2011.

Ohlin, B., "The reparation problem: a discussion," Economic Journal, 1929, 39 (154), 172182.

Ramey, Valerie A., "Can Government Purchases Stimulate the Ecnomy?," Journal of Economic Literature, 2011, 49 (3), 673-685.

Serrato, J. C. S. and P. Wingender, "Estimating Local Multipliers," University of California at Berkeley Working Paper 2010. 
Shoag, Danny, "The Impact of Government Spending Shocks: Evidence on the Multiplier from State Pension Plan Returns," Harvard University Working Paper 2010.

Werning, Ivan, "Managing a Liquidity Trap: Monetary and Fiscal Policy," 2012. NBER Working Papers.

Woodford, Michael, "Simple Analytics of the Government Expenditure Multiplier," American Economic Journal: Macroeconomics, January 2011, 3 (1), 1-35.

\section{A Proof of Proposition 1}

We need to solve the system

$$
\begin{aligned}
\dot{c}_{t} & =\hat{\sigma}^{-1}\left(i_{t}-\pi_{t}-r_{t}\right), \\
\dot{\pi}_{t} & =\rho \pi_{t}-\kappa\left(c_{t}+(1-\Gamma) g_{t}\right) .
\end{aligned}
$$

Equivalently

$$
\ddot{c}_{t}-\rho \dot{c}_{t}-\hat{\sigma}^{-1} \mathcal{K}\left(c_{t}+(1-\Gamma) g_{t}\right)=0 .
$$

which has characteristic roots

$$
\bar{v}=\frac{\rho+\sqrt{\rho^{2}+4 \hat{\sigma}^{-1} \mathcal{K}}}{2} \quad v=\frac{\rho-\sqrt{\rho^{2}+4 \hat{\sigma}^{-1} \mathcal{K}}}{2} .
$$

Based on these we make the guess that

$$
c_{t}=A \int_{t} e^{-\bar{v}(z-t)} g_{z} d z-A \int_{t} e^{-v(z-t)} g_{z} d z
$$

for some constant $A$ and verify that

$$
\begin{aligned}
\dot{c}_{t} & =-A g_{t}+\bar{v} A \int_{t} e^{-\bar{v}(z-t)} g_{z} d z+A g_{t}-v A \int_{t} e^{-v(z-t)} g_{z} d z, \\
& =\bar{v} A \int_{t} e^{-\bar{v}(z-t)} g_{z} d z-v A \int_{t} e^{-v(z-t)} g_{z} d z, \\
\ddot{c}_{t} & =\bar{v}^{2} A \int_{t} e^{-\bar{v}(z-t)} g_{z} d s-v^{2} A \int_{t} e^{-\bar{v}(z-t)} g_{z} d s+(-\bar{v}+v) A g_{t} .
\end{aligned}
$$

This implies that

$$
\ddot{c}_{t}-\rho \dot{c}_{t}-\sigma^{-1} \mathcal{K}\left(c_{t}+(1-\Gamma) g_{t}\right)=\left((-\bar{v}+v) A-\sigma^{-1} \mathcal{K}(1-\Gamma)\right) g_{t}=0 .
$$


if and only if

$$
A=\frac{\hat{\sigma}^{-1} \mathcal{K}(1-\Gamma)}{(-\bar{v}+v)}=-\frac{\hat{\sigma}^{-1} \mathcal{\kappa}(1-\Gamma)}{\sqrt{\rho^{2}+4 \sigma^{-1} \mathcal{K}}} .
$$

Hence we can write

$$
c_{t}=\frac{\hat{\sigma}^{-1} \mathcal{K}(1-\Gamma)}{\sqrt{\rho^{2}+4 \sigma^{-1} \mathcal{K}}} \int_{0}^{\infty}\left(e^{(\bar{v}-v) s}-1\right) e^{-\bar{v} s} g_{t+s} d s
$$

To characterize the properties of the multiplier write

$$
\alpha_{s}^{c}=\frac{\left(\Delta^{2}-\rho^{2}\right)(1-\Gamma)}{4 \Delta}\left(e^{\Delta s}-1\right) e^{-\frac{\rho+\Delta}{2} s},
$$

where $\Delta=\sqrt{\rho^{2}+4 \hat{\sigma}^{-1} \kappa}$, the claims that $\alpha_{0}^{c}=0$ and $\alpha_{s}^{c}>0$ for $s>0$ are immediate.

The first derivative is

$$
\begin{aligned}
\frac{\partial \alpha_{s}^{c}}{\partial s} & =\frac{\left(\Delta^{2}-\rho^{2}\right)(1-\Gamma)}{4 \Delta}\left(\Delta e^{\Delta s} e^{-\frac{\rho+\Delta}{2} s}-\frac{\rho+\Delta}{2}\left(e^{\Delta s}-1\right) e^{-\frac{\rho+\Delta}{2} s}\right), \\
& =\frac{\left(\Delta^{2}-\rho^{2}\right)(1-\Gamma)}{4 \Delta}\left(\frac{\rho+\Delta}{2}+\frac{\Delta-\rho}{2} e^{\Delta s}\right) e^{-\frac{\rho+\Delta}{2} s}>0,
\end{aligned}
$$

since $\Delta>\rho$ as long as $\kappa>0$. The second derivative is

$$
\begin{aligned}
\frac{\partial^{2} \alpha_{s}^{c}}{\partial s^{2}}(s) & =e^{-\frac{\rho+\Delta}{2} s}\left(-\frac{\rho+\Delta}{2}\left(\frac{\rho+\Delta}{2}+\frac{\Delta-\rho}{2} e^{\Delta s}\right)+\Delta \frac{\Delta-\rho}{2} e^{\Delta s}\right) \\
& =e^{-\frac{\rho+\Delta}{2} s}\left(-\left(\frac{\rho+\Delta}{2}\right)^{2}+\left(\frac{\Delta-\rho}{2}\right)^{2} e^{\Delta s}\right)>0 .
\end{aligned}
$$

Define $\bar{s}$ by the condition that

$$
-\left(\frac{\rho+\Delta}{2}\right)^{2}+\left(\frac{\Delta-\rho}{2}\right)^{2} e^{\Delta \bar{s}}=0
$$

then $\alpha_{s}^{c}$ is locally strictly convex for all $s \geq \bar{s}$. It follows that $\alpha_{s}^{c}$ is unbounded.

\section{B Proof of Proposition 2}

The two limits are immediate. To establish monotonicity, we use brute force to compute 


$$
\begin{aligned}
\frac{\partial \alpha_{s}^{c}}{\partial \Delta}=\frac{(1-\Gamma)}{2}\left(e^{\Delta s}-1\right) e^{-\frac{\rho+\Delta}{2} s}-\frac{\left(\Delta^{2}-\rho^{2}\right)(1-\Gamma)}{4 \Delta^{2}}\left(e^{\Delta s}-1\right) e^{-\frac{\rho+\Delta}{2} s} & \\
& +\frac{\left(\Delta^{2}-\rho^{2}\right)(1-\Gamma)}{4 \Delta} s e^{\Delta s} e^{-\frac{\rho+\Delta}{2} s} \\
& \quad+\frac{\left(\Delta^{2}-\rho^{2}\right)(1-\Gamma)}{4 \Delta}\left(e^{\Delta s}-1\right) e^{-\frac{\rho+\Delta}{2} s}\left(-\frac{1}{2} s\right) .
\end{aligned}
$$

Rearranging we get

$$
\begin{aligned}
e^{\frac{\rho+\Delta}{2} s} \frac{\partial \alpha_{s}^{c}}{\partial \Delta}=\frac{1}{2}\left(1-\frac{\Delta^{2}-\rho^{2}}{2 \Delta^{2}}\right)(1-\Gamma) & \left(e^{\Delta s}-1\right) \\
& +\frac{1}{2} \frac{\left(\Delta^{2}-\rho^{2}\right)(1-\Gamma)}{4 \Delta} s e^{\Delta s}+\frac{\left(\Delta^{2}-\rho^{2}\right)(1-\Gamma)}{4 \Delta}
\end{aligned}
$$

so that all terms are positive.

\section{Proof of Proposition 4}

With complete markets, $\theta=0$. Imagine an experiment where $i_{t}^{*}=\rho, g_{t}^{*}=y_{t}^{*}=c_{t}^{*}=0$ $=0$. If $g_{t}=0$ throughout then $y_{t}=c_{t}=0$. The system becomes

$$
\begin{gathered}
\dot{\pi}_{H, t}=\rho \pi_{H, t}-\kappa\left[c_{t}+(1-\xi) g_{t}\right], \\
\dot{c}_{t}=-\hat{\sigma}^{-1} \pi_{H, t}, \\
c_{0}=0 .
\end{gathered}
$$

Let $X_{t}=\left[\pi_{H, t}, c_{t}\right]^{\prime}, B_{t}=\left[-\kappa(1-\xi) g_{t}, 0\right]^{\prime}=-\kappa(1-\xi) g_{t} E_{1}$ and $A=\left[\begin{array}{cc}\rho & -\kappa \\ -\hat{\sigma}^{-1} & 0\end{array}\right]$. We have $\dot{X}_{t}=A X_{t}+B_{t}$. The matrix $A$ has one positive and one negative eigenvalue. The negative eigenvalue is given by $v=\frac{\rho-\sqrt{\rho^{2}+4 \kappa \hat{\sigma}^{-1}}}{2}$. The associated eigenvector is $X_{v}=[-v \hat{\sigma}, 1]^{\prime}$. The solution is

$$
X_{t}=\alpha_{v} e^{v t} X_{v}+\kappa(1-\xi) \int_{t}^{\infty} g_{s} e^{-A(s-t)} E_{1} d s
$$

where $X_{0}$ and $\alpha_{v}$ solve the system of three equations in three unknowns

$$
X_{0}-\kappa(1-\xi) \int_{0}^{\infty} g_{t} e^{-A t} E_{1} d t=\alpha_{v} X_{v}
$$




$$
E_{2}^{\prime} X_{0}=(1-\mathcal{G})(1-\alpha) \theta
$$

The solution is

$$
\begin{gathered}
\alpha_{v}=-\kappa(1-\xi) \int_{0}^{\infty} E_{2}^{\prime} g_{t} e^{-A t} E_{1} d t \\
\pi_{H, 0}=\kappa(1-\xi) \int_{0}^{\infty} g_{t} E_{1}^{\prime} e^{-A t} E_{1} d t+v \hat{\sigma} \kappa(1-\xi) \int_{0}^{\infty} E_{2}^{\prime} g_{t} e^{-A t} E_{1} d t \\
c_{0}=0 .
\end{gathered}
$$

Hence we have

$$
\begin{gathered}
c_{t}=-e^{v t} \kappa(1-\xi) \int_{0}^{\infty} g_{t} E_{2}^{\prime} e^{-A t} E_{1} d t+\kappa(1-\xi) \int_{t}^{\infty} g_{s} E_{2}^{\prime} e^{-A(s-t)} E_{1} d s, \\
\pi_{H, t}=v \hat{\sigma} e^{v t} \kappa(1-\xi) \int_{0}^{\infty} g_{t} E_{2}^{\prime} e^{-A t} E_{1} d t+\kappa(1-\xi) \int_{t}^{\infty} g_{s} E_{1}^{\prime} e^{-A(s-t)} E_{1} d s .
\end{gathered}
$$

Denote the positive eigenvalue of $A$ by $\bar{v}=\frac{\rho+\sqrt{\rho^{2}+4 \kappa \hat{\sigma}^{-1}}}{2}$ and the corresponding eigenvector by $X_{\bar{v}}=[-\bar{v} \hat{\sigma}, 1]^{\prime}$. We have $E_{1}=\frac{1}{(\bar{v}-v) \hat{\sigma}}\left(X_{v}-X_{\bar{v}}\right)$. Hence we can write

$$
\begin{aligned}
& c_{t}=-e^{v t} \kappa(1-\xi) \int_{0}^{\infty} g_{t} E_{2}^{\prime} e^{-A t} \frac{1}{(\bar{v}-v) \hat{\sigma}}\left(X_{v}-X_{\bar{v}}\right) d t \\
& \quad+\kappa(1-\xi) \int_{t}^{\infty} g_{s} E_{2}^{\prime} e^{-A(s-t)} \frac{1}{(\bar{v}-v) \hat{\sigma}}\left(X_{v}-X_{\bar{v}}\right) d s, \\
& \pi_{H, t}=v \hat{\sigma} e^{v t} \kappa(1-\xi) \int_{0}^{\infty} g_{t} E_{2}^{\prime} e^{-A t} \frac{1}{(\bar{v}-v) \hat{\sigma}}\left(X_{v}-X_{\bar{v}) d t}\right. \\
& \quad+\kappa(1-\xi) \int_{t}^{\infty} g_{s} E_{1}^{\prime} e^{-A(s-t)} \frac{1}{(\bar{v}-v) \hat{\sigma}}\left(X_{v}-X_{\bar{v}}\right) d s .
\end{aligned}
$$

We get

$$
\begin{gathered}
c_{t}=-e^{v t} \kappa(1-\xi) \int_{0}^{\infty} g_{t} \frac{1}{\hat{\sigma}} \frac{e^{-v t}-e^{-\bar{v} t}}{\bar{v}-v} d t+\kappa(1-\xi) \int_{t}^{\infty} g_{s} \frac{1}{\hat{\sigma}} \frac{e^{-v(s-t)}-e^{-\bar{v}(s-t)}}{\bar{v}-v} d s, \\
\pi_{H, t}=v \hat{\sigma} e^{v t} \kappa(1-\xi) \int_{0}^{\infty} g_{t} \frac{1}{\hat{\sigma}} \frac{e^{-v t}-e^{-\bar{v} t}}{\bar{v}-v} d t+\kappa(1-\xi) \int_{t}^{\infty} g_{s} \frac{v e^{-v(s-t)}-\bar{v} e^{-\bar{v}(s-t)}}{v-\bar{v}} d s .
\end{gathered}
$$

Now suppose that $g_{t}=g e^{-\rho_{g} t}$. Then

$$
c_{t}=-e^{v t}\left(1-e^{-\left(v+\rho_{g}\right) t}\right) \kappa(1-\xi) g\left[E_{2}^{\prime}\left(A+\rho_{g} I\right)^{-1} E_{1}\right]
$$


where

$$
\begin{gathered}
A+\rho_{g} I=\left[\begin{array}{cc}
\rho+\rho_{g} & -\kappa \\
-\hat{\sigma}^{-1} & \rho_{g}
\end{array}\right], \\
\left(A+\rho_{g} I\right)^{-1}=\frac{1}{\rho_{g}\left(\rho+\rho_{g}\right)-\kappa \hat{\sigma}^{-1}}\left[\begin{array}{cc}
\rho_{g} & \kappa \\
\hat{\sigma}^{-1} & \rho+\rho_{g}
\end{array}\right],
\end{gathered}
$$

so that

$$
E_{2}^{\prime}\left(A+\rho_{g} I\right)^{-1} E_{1}=\frac{\hat{\sigma}^{-1}}{\rho_{g}\left(\rho+\rho_{g}\right)-\kappa \hat{\sigma}^{-1}} .
$$

\section{Proof of Proposition 6}

Let $X_{t}=\left[\pi_{H, t}, c_{t}\right]^{\prime}, B_{t}=\left[-\kappa(1-\xi) g_{t}-(1-\mathcal{G}) \lambda \hat{\sigma} \alpha \omega \theta, 0\right]^{\prime}=-\kappa(1-\xi) g_{t} E_{1}-(1-$ $\mathcal{G}) \lambda \hat{\sigma} \alpha \omega \theta E_{1}$ and $A=\left[\begin{array}{cc}\rho & -\kappa \\ -\hat{\sigma}^{-1} & 0\end{array}\right]$. We have $\dot{X}_{t}=A X_{t}+B_{t}$. The matrix $A$ has one positive and one negative eigenvalue. The negative eigenvalue is given by $v=\frac{\rho-\sqrt{\rho^{2}+4 \kappa \hat{\sigma}^{-1}}}{2}$. The associated eigenvector is $X_{v}=[-v \hat{\sigma}, 1]^{\prime}$. The solution is

$$
X_{t}=\alpha_{v} e^{\nu t} X_{v}+\kappa(1-\xi) \int_{t}^{\infty} g_{s} e^{-A(s-t)} E_{1} d s+(1-\mathcal{G}) \lambda \hat{\sigma} \alpha \omega \theta A^{-1} E_{1}
$$

where $X_{0}, \alpha_{v}$ and $\theta$ solve the system of four equations in four unknowns

$$
\begin{gathered}
X_{0}-\kappa(1-\xi) \int_{0}^{\infty} g_{t} e^{-A t} E_{1} d t-(1-\mathcal{G}) \lambda \hat{\sigma} \alpha \omega \theta A^{-1} E_{1}=\alpha_{v} X_{v} \\
E_{2}^{\prime} X_{0}=(1-\mathcal{G})(1-\alpha) \theta \\
\theta=\Omega\left[\frac{\rho}{\rho-v} \alpha_{v}+\theta(1-\mathcal{G}) \lambda \hat{\sigma} \alpha \omega E_{2}^{\prime} A^{-1} E_{1}+\kappa(1-\xi) \rho \int_{0}^{\infty} g_{s} e^{-\rho s} E_{2}^{\prime}(A-\rho I)^{-1}\left(1-e^{-(A-\rho I) s}\right) E_{1} d s\right] .
\end{gathered}
$$

We find

$$
\begin{aligned}
& \theta\left[(1-\mathcal{G})(1-\alpha)-(1-\mathcal{G}) \lambda \hat{\sigma} \alpha \omega E_{2}^{\prime} A^{-1} E_{1}\right]-\kappa(1-\xi) \int_{0}^{\infty} g_{t} E_{2}^{\prime} e^{-A t} E_{1} d t=\alpha_{v}, \\
\theta= & \Omega\left[\frac{\rho}{\rho-v} \alpha_{v}+\theta(1-\mathcal{G}) \lambda \hat{\sigma} \alpha \omega E_{2}^{\prime} A^{-1} E_{1}+\kappa(1-\xi) \rho \int_{0}^{\infty} g_{s} e^{-\rho s} E_{2}^{\prime}(A-\rho I)^{-1}\left(1-e^{-(A-\rho I) s}\right) E_{1} d s\right] .
\end{aligned}
$$

We then have

$$
c_{t}=-e^{\nu t} \kappa(1-\xi) \int_{0}^{\infty} g_{t} E_{2}^{\prime} e^{-A t} E_{1} d t+\kappa(1-\xi) \int_{t}^{\infty} g_{s} E_{2}^{\prime} e^{-A(s-t)} E_{1} d s
$$




$$
\begin{array}{r}
+\theta\left[(1-\mathcal{G})(1-\alpha) e^{v t}-\left(1-e^{\nu t}\right)(1-\mathcal{G}) \hat{\sigma} \frac{\alpha \omega}{1+\phi}\right], \\
\theta=\frac{\Omega \mathcal{K}(1-\xi)\left[-\frac{\rho}{\rho-\nu} \int_{0}^{\infty} g_{t} E_{2}^{\prime} e^{-A t} E_{1} d t+\rho \int_{0}^{\infty} g_{s} e^{-\rho s} E_{2}^{\prime}(A-\rho I)^{-1}\left(1-e^{-(A-\rho I) s}\right) E_{1} d s\right]}{1-\Omega\left[\frac{\rho}{\rho-\nu}(1-\mathcal{G})(1-\alpha)+\frac{v}{\rho-\nu}(1-\mathcal{G}) \hat{\sigma} \frac{\alpha \omega}{1+\phi}\right]} .
\end{array}
$$

Recall that $E_{1}=\frac{1}{(\bar{v}-v) \hat{\sigma}}\left(X_{v}-X_{\bar{v}}\right)$. We can therefore rewrite

$$
\begin{aligned}
& c_{t}=-e^{v t} \kappa(1-\xi) \int_{0}^{\infty} g_{t} \frac{1}{\hat{\sigma}} \frac{e^{-v t}-e^{-\bar{v} t}}{\bar{v}-v} d t+\kappa(1-\xi) \int_{t}^{\infty} g_{s} \frac{1}{\hat{\sigma}} \frac{e^{-v(s-t)}-e^{-\bar{v}(s-t)}}{\bar{v}-v} d s, \\
& +\theta\left[(1-\mathcal{G})(1-\alpha) e^{\nu t}-\left(1-e^{\nu t}\right)(1-\mathcal{G}) \hat{\sigma} \frac{\alpha \omega}{1+\phi}\right], \\
& \theta=\frac{\Omega \kappa(1-\xi)\left[-\frac{\rho}{\rho-\nu} \int_{0}^{\infty} g_{t} \hat{\sigma}^{-1}\left(\frac{e^{-v t}-e^{-\bar{v} t}}{\bar{v}-v}\right) d t+\rho \int_{0}^{\infty} g_{s} e^{-\rho s} \hat{\sigma}^{-1} \frac{\frac{1-e^{-(\nu-\rho) s}}{v-\rho}-\frac{1-e^{-(\bar{v}-\rho) s}}{\bar{v}-\rho}}{\bar{v}-v} d s\right]}{1-\Omega\left[\frac{\rho}{\rho-\nu}(1-\mathcal{G})(1-\alpha)+\frac{v}{\rho-v}(1-\mathcal{G}) \hat{\sigma} \frac{\alpha \omega}{1+\phi}\right]} .
\end{aligned}
$$

Suppose that $g_{t}=g e^{-\rho_{g} t}$. We can solve $\alpha_{v}$ and $\theta$ as the solution of the following system of two equations in two unknowns

$$
\begin{gathered}
\alpha_{v}+\theta\left[(1-\mathcal{G}) \lambda \hat{\sigma} \alpha \omega E_{2}^{\prime} A^{-1} E_{1}-(1-\mathcal{G})(1-\alpha)\right]=-g \kappa(1-\xi) E_{2}^{\prime}\left(A+\rho_{g} I\right)^{-1} E_{1} \\
-\alpha_{v} \frac{\left(\frac{\omega}{\sigma}-1\right) \hat{\sigma}}{1+(1-\mathcal{G})\left(\frac{\omega}{\sigma}-1\right) \hat{\sigma}(1-\alpha)} \frac{\rho}{\rho-v} E_{2}^{\prime} X_{v}+\theta\left[1-\frac{(1-\mathcal{G})\left(\frac{\omega}{\sigma}-1\right) \hat{\sigma}}{1+(1-\mathcal{G})\left(\frac{\omega}{\sigma}-1\right) \hat{\sigma}(1-\alpha)} \lambda \hat{\sigma} \alpha \omega E_{2}^{\prime} A^{-1} E_{1}\right] \\
=\frac{\left(\frac{\omega}{\sigma}-1\right) \hat{\sigma}}{1+(1-\mathcal{G})\left(\frac{\omega}{\sigma}-1\right) \hat{\sigma}(1-\alpha)}\left[\kappa(1-\xi) g \frac{\rho}{\rho+\rho_{g}} E_{2}^{\prime}\left(A+\rho_{g} I\right)^{-1} E_{1}\right] .
\end{gathered}
$$

where

$$
E_{2}^{\prime}\left(A+\rho_{g} I\right)^{-1} E_{1}=\frac{\hat{\sigma}^{-1}}{\rho_{g}\left(\rho+\rho_{g}\right)-\kappa \hat{\sigma}^{-1}},
$$

which is of the same sign as $v+\rho_{g}$. Hence we get that $\theta$ is of the same sign as $1-\frac{\omega}{\sigma}$ (for $\frac{\omega}{\sigma}$ close to 1$)$ where $\omega=\sigma \gamma+(1-\alpha)(\sigma \eta-1)$. And then we can solve

$$
\begin{aligned}
c_{t}=\left[e^{\nu t}(1-\mathcal{G})(1-\alpha)+\left(1-e^{\nu t}\right)(1-\mathcal{G}) \lambda \hat{\sigma} \alpha \omega E_{2}^{\prime} A^{-1} E_{1}\right] & \theta \\
& -e^{\nu t}\left(1-e^{-\left(v+\rho_{g}\right) t}\right) g \kappa(1-\xi) E_{2}^{\prime}\left(A+\rho_{g} I\right)^{-1} E_{1} .
\end{aligned}
$$




\section{E Proof of Proposition 8}

We have

$$
\mathrm{nfa}_{0}=\int_{0}^{\infty} e^{-\rho t} g_{t} d t
$$

The system is

$$
\begin{gathered}
\dot{\pi}_{H, t}=\rho \pi_{H, t}-\kappa\left[c_{t}+(1-\xi) g_{t}\right]-(1-\mathcal{G}) \lambda \hat{\sigma} \alpha \omega \theta, \\
\dot{c}_{t}=-\hat{\sigma}^{-1} \pi_{H, t} \\
\mathcal{c}_{0}=(1-\mathcal{G})(1-\alpha) \theta, \\
\theta=\frac{\rho}{\alpha} \frac{1}{1-\mathcal{G}} \frac{1}{\Gamma} \int_{0}^{\infty} e^{-\rho t} g_{t} d t+\Omega \rho \int_{0}^{\infty} e^{-\rho t} c_{t} d t .
\end{gathered}
$$

The solution is

$$
X_{t}=\alpha_{v} e^{v t} X_{v}+(1-\mathcal{G}) \lambda \hat{\sigma} \alpha \omega \theta A^{-1} E_{1}+\kappa(1-\xi) \int_{t}^{\infty} g_{s} e^{-A(s-t)} E_{1} d s,
$$

where $X_{0}$ and $\alpha_{v}$ solve the system of three equations in three unknowns

$$
\begin{gathered}
X_{0}-(1-\mathcal{G}) \lambda \hat{\sigma} \alpha \omega \theta A^{-1} E_{1}-\kappa(1-\xi) \int_{0}^{\infty} g_{t} e^{-A t} E_{1} d t=\alpha_{v} X_{v} \\
E_{2}^{\prime} X_{0}=(1-\mathcal{G})(1-\alpha) \theta \\
\theta=\frac{\rho}{\alpha} \frac{1}{1-\mathcal{G}} \frac{1}{\Gamma} \int_{0}^{\infty} e^{-\rho t} g_{t} d t \\
+\Omega\left[\frac{\rho}{\rho+v} \alpha_{v}+(1-\mathcal{G}) \lambda \hat{\sigma} \alpha \omega \theta E_{2}^{\prime} A^{-1} E_{1}\right. \\
\left.+\kappa(1-\xi) \int_{0}^{\infty} g_{t} E_{2}^{\prime} e^{-A t} E_{1} d t-\kappa(1-\xi) \int_{0}^{\infty} g_{t} e^{-\rho t} E_{2}^{\prime} e^{-A t} E_{1} d t\right] .
\end{gathered}
$$

The Cole Obstfeld case. In the Cole-Obstfeld case, we get

$$
\begin{aligned}
\alpha_{v} & =\left[(1-\mathcal{G})(1-\alpha)-\lambda \alpha E_{2}^{\prime} A^{-1} E_{1}\right] \frac{\rho}{\alpha} \frac{1}{1-\mathcal{G}} \int_{0}^{\infty} e^{-\rho t} g_{t} d t-\kappa(1-\xi) \int_{0}^{\infty} g_{t} E_{2}^{\prime} e^{-A t} E_{1} d t, \\
c_{t} & =\left[\frac{\rho}{\alpha} \frac{1}{1-\mathcal{G}} \int_{0}^{\infty} e^{-\rho t} g_{t} d t\right]\left[(1-\mathcal{G})(1-\alpha) e^{v t}+\left(1-e^{v t}\right) \lambda \alpha E_{2}^{\prime} A^{-1} E_{1}\right]
\end{aligned}
$$




$$
-e^{\nu t} \kappa(1-\xi) \int_{0}^{\infty} g_{t} E_{2}^{\prime} e^{-A t} E_{1} d t+\kappa(1-\xi) \int_{t}^{\infty} g_{s} E_{2}^{\prime} e^{-A(s-t)} E_{1} d s
$$

We now specialize to $g_{t}=g e^{-\rho_{g} t}$. We find

$$
\begin{gathered}
\alpha_{v}=\left[(1-\mathcal{G})(1-\alpha)-\lambda \alpha E_{2}^{\prime} A^{-1} E_{1}\right] \frac{\rho}{\rho+\rho_{g}} \frac{1}{\alpha} \frac{1}{1-\mathcal{G}} g-\kappa(1-\xi) g E_{2}^{\prime}\left(A+\rho_{g} I\right)^{-1} E_{1}, \\
c_{t}=g\left[e^{\nu t}(1-\mathcal{G})(1-\alpha)-\left(1-e^{\nu t}\right) \frac{\lambda \alpha}{\kappa}\right] \frac{1}{1-\mathcal{G}} \frac{1}{\alpha} \frac{\rho}{\rho+\rho_{g}}-g e^{\nu t}\left(\frac{1-e^{-\left(v+\rho_{g}\right) t}}{\nu+\rho_{g}}\right) \kappa(1-\xi) \frac{1-\mathcal{G}}{\bar{v}+\rho_{g}} .
\end{gathered}
$$

We see that $c_{0}=g \frac{1-\alpha}{\alpha} \frac{\rho}{\rho+\rho_{g}}$ and $\lim _{t \rightarrow \infty} \mathcal{C}_{t}=-g \frac{1}{1-\mathcal{G}} \frac{\lambda}{\kappa} \frac{\rho}{\rho+\rho_{g}}$. Hence in the case of a stimulus $g>0$, we have $c_{0}>0>\lim _{t \rightarrow \infty} c_{t}$.

\section{F Proof of Proposition 9}

Here we assume that prices are entirely rigid. The system becomes

$$
\begin{gathered}
\dot{c}_{t}=0, \\
c_{0}=(1-\mathcal{G})(1-\alpha) \theta \\
\theta=\frac{\rho}{\alpha} \frac{1}{1-\mathcal{G}} \frac{1}{\Gamma} \int_{0}^{\infty} e^{-\rho t} g_{t} d t+\Omega \rho \int_{0}^{\infty} e^{-\rho t} c_{t} d t .
\end{gathered}
$$

The solution is

$$
\begin{aligned}
c_{t} & =\frac{(1-\mathcal{G})(1-\alpha)}{1-\Omega(1-\mathcal{G})(1-\alpha)} \frac{\rho}{\alpha} \frac{1}{1-\mathcal{G}} \frac{1}{\Gamma} \int_{0}^{\infty} e^{-\rho s} g_{s} d s, \\
\theta & =\frac{1}{1-\Omega(1-\mathcal{G})(1-\alpha)} \frac{\rho}{\alpha} \frac{1}{1-\mathcal{G}} \frac{1}{\Gamma} \int_{0}^{\infty} e^{-\rho t} g_{t} d t .
\end{aligned}
$$

In the special case where $g_{t}=g e^{-\rho_{g} t}$, we get

$$
\begin{aligned}
c_{t} & =\frac{(1-\mathcal{G})(1-\alpha)}{1-\Omega(1-\mathcal{G})(1-\alpha)} \frac{1}{\alpha} \frac{1}{1-\mathcal{G}} \frac{1}{\Gamma} \frac{\rho}{\rho+\rho_{g}} g, \\
\theta & =\frac{1}{1-\Omega(1-\mathcal{G})(1-\alpha)} \frac{1}{\alpha} \frac{1}{1-\mathcal{G}} \frac{1}{\Gamma} \frac{\rho}{\rho+\rho_{g}} g .
\end{aligned}
$$




\section{G Closed Form Solution for Government Spending Paid by Foreign, General Case}

We specialize to $g_{t}=g e^{-\rho_{g} t}$. The system becomes

$$
\begin{gathered}
X_{0}-(1-\mathcal{G}) \lambda \hat{\sigma} \alpha \omega \theta A^{-1} E_{1}-\kappa(1-\xi) g\left(A+\rho_{g} I\right)^{-1} E_{1}=\alpha_{v} X_{v}, \\
E_{2}^{\prime} X_{0}=(1-\mathcal{G})(1-\alpha) \theta \\
\theta=\frac{\rho}{\rho+\rho_{g}} \frac{1}{\alpha} \frac{1}{1-\mathcal{G}} \frac{1}{\Gamma} g \\
+\Omega\left[\frac{\rho}{\rho+v} \alpha_{v}+(1-\mathcal{G}) \lambda \hat{\sigma} \alpha \omega \theta E_{2}^{\prime} A^{-1} E_{1}\right. \\
\left.+\kappa(1-\xi) g E_{2}^{\prime}\left(A+\rho_{g} I\right)^{-1} E_{1}-\kappa(1-\xi) E_{2}^{\prime} g\left(A+\left(\rho+\rho_{g}\right) I\right)^{-1} E\right]_{1} .
\end{gathered}
$$

The solution is

$$
\begin{gathered}
\alpha_{v}=(1-\mathcal{G})(1-\alpha) \theta\left[1-\frac{\alpha}{1-\alpha} \lambda \hat{\sigma} \omega E_{2}^{\prime} A^{-1} E_{1}\right]-\kappa(1-\xi) g E_{2}^{\prime}\left(A+\rho_{g} I\right)^{-1} E_{1}, \\
\theta=\frac{\rho}{\rho+\rho_{g}} \frac{1}{\alpha} \frac{1}{1-\mathcal{G}} \frac{1}{\Gamma} g+\Omega\left[\frac{\rho}{\rho+v} \alpha_{v}+(1-\mathcal{G}) \lambda \hat{\sigma} \alpha \omega \theta E_{2}^{\prime} A^{-1} E_{1}\right. \\
\left.+\kappa(1-\xi) g E_{2}^{\prime}\left(A+\rho_{g} I\right)^{-1} E_{1}-\kappa(1-\xi) E_{2}^{\prime} g\left(A+\left(\rho+\rho_{g}\right) I\right)^{-1} E_{1}\right] .
\end{gathered}
$$

We can solve further. Define

$$
\begin{gathered}
\tilde{\Gamma}=1-\Omega(1-\mathcal{G}) \lambda \hat{\sigma} \alpha \omega \theta E_{2}^{\prime} A^{-1} E_{1} \\
\hat{\Gamma}=\left[1-(1-\mathcal{G})(1-\alpha)\left[1-\frac{\alpha}{1-\alpha} \lambda \hat{\sigma} \omega E_{2}^{\prime} A^{-1} E_{1}\right] \frac{1}{\tilde{\Gamma}} \Omega \frac{\rho}{\rho+v}\right],
\end{gathered}
$$

and

$$
\begin{array}{r}
\hat{\Omega}=(1-\mathcal{G})(1-\alpha)\left[1-\frac{\alpha}{1-\alpha} \lambda \hat{\sigma} \omega E_{2}^{\prime} A^{-1} E_{1}\right] \frac{1}{\tilde{\Gamma}} \frac{1}{\hat{\Gamma}} \Omega \kappa(1-\xi)\left[E_{2}^{\prime}\left(A+\rho_{g} I\right)^{-1} E_{1}\right. \\
\left.-E_{2}^{\prime}\left(A+\left(\rho+\rho_{g}\right) I\right)^{-1} E_{1}\right],
\end{array}
$$




$$
\begin{aligned}
& \tilde{\Omega}=\frac{\rho}{\rho+\rho_{g}} \frac{1}{\alpha} \frac{1}{1-\mathcal{G}}\left[\frac{1}{\Gamma}\right.\left.\frac{1}{\tilde{\Gamma}}-1\right] \\
&+\frac{1}{\tilde{\Gamma}} \Omega \frac{\rho}{\rho+v}\left[-\frac{1}{\hat{\Gamma}} \kappa(1-\xi) E_{2}^{\prime}\left(A+\rho_{g}\right)^{-1} E_{1}\right. \\
&\left.+(1-\mathcal{G})(1-\alpha)\left[1-\frac{\alpha}{1-\alpha} \lambda \hat{\sigma} \omega E_{2}^{\prime} A^{-1} E_{1}\right] \frac{\rho}{\rho+\rho_{g}} \frac{1}{\alpha} \frac{1}{1-\mathcal{G}} \frac{1}{\Gamma \tilde{\Gamma} \hat{\Gamma}}+\hat{\Omega}\right] \\
&+\kappa(1-\xi) \frac{1}{\tilde{\Gamma}} \Omega\left[E_{2}^{\prime}\left(A+\rho_{g} I\right)^{-1} E_{1}-E_{2}^{\prime} g\left(A+\left(\rho+\rho_{g}\right) I\right)^{-1} E_{1}\right],
\end{aligned}
$$

and

$$
\begin{aligned}
& \overline{\bar{\Omega}}=\left[\frac{1}{\Gamma \tilde{\Gamma} \hat{\Gamma}}-1\right](1-\mathcal{G})(1-\alpha)\left[1-\frac{\alpha}{1-\alpha} \lambda \hat{\sigma} \omega E_{2}^{\prime} A^{-1} E_{1}\right] \frac{\rho}{\rho+\rho_{g}} \frac{1}{\alpha} \frac{1}{1-\mathcal{G}} \\
& +\hat{\Omega}-\left(\frac{1}{\hat{\Gamma}}-1\right) \kappa(1-\xi) E_{2}^{\prime}\left(A+\rho_{g}\right)^{-1} E_{1} .
\end{aligned}
$$

We have

$$
\begin{aligned}
& \theta=\frac{1}{\tilde{\Gamma}} \frac{\rho}{\rho+\rho_{g}} \frac{1}{\alpha} \frac{1}{1-\mathcal{G}} \frac{1}{\Gamma^{g}} \\
& +\frac{1}{\tilde{\Gamma}} \Omega\left[\frac{\rho}{\rho+v} \alpha_{v}+\kappa(1-\xi) g E_{2}^{\prime}\left(A+\rho_{g} I\right)^{-1} E_{1}-\kappa(1-\xi) E_{2}^{\prime} g\left(A+\left(\rho+\rho_{g}\right) I\right)^{-1} E_{1}\right], \\
& \alpha_{v}=-\kappa(1-\xi) g E_{2}^{\prime}\left(A+\rho_{g} I\right)^{-1} E_{1} \\
& \quad+(1-\mathcal{G})(1-\alpha)\left[1-\frac{\alpha}{1-\alpha} \lambda \hat{\sigma} \omega E_{2}^{\prime} A^{-1} E_{1}\right] \frac{1}{\tilde{\Gamma}} \frac{\rho}{\rho+\rho_{g}} \frac{1}{\alpha} \frac{1}{1-\mathcal{G}} \frac{1}{\Gamma} g \\
& \quad+(1-\mathcal{G})(1-\alpha)\left[1-\frac{\alpha}{1-\alpha} \lambda \hat{\sigma} \omega E_{2}^{\prime} A^{-1} E_{1}\right] \frac{1}{\tilde{\Gamma}} \Omega\left[\frac{\rho}{\rho+v} \alpha_{v}\right. \\
& \left.\quad+\kappa(1-\xi) g E_{2}^{\prime}\left(A+\rho_{g} I\right)^{-1} E_{1}-\kappa(1-\xi) E_{2}^{\prime} g\left(A+\left(\rho+\rho_{g}\right) I\right)^{-1} E_{1}\right] .
\end{aligned}
$$

We find

$$
\begin{aligned}
& \alpha_{v}=-g \frac{1}{\hat{\Gamma}} \kappa(1-\xi) E_{2}^{\prime}\left(A+\rho_{g}\right)^{-1} E_{1} \\
& \quad+g(1-\mathcal{G})(1-\alpha)\left[1-\frac{\alpha}{1-\alpha} \lambda \hat{\sigma} \omega E_{2}^{\prime} A^{-1} E_{1}\right] \frac{\rho}{\rho+\rho_{g}} \frac{1}{\alpha} \frac{1}{1-\mathcal{G}} \frac{1}{\Gamma \tilde{\Gamma} \hat{\Gamma}}+g \hat{\Omega} .
\end{aligned}
$$


We can now replace to find $\theta$ :

$$
\begin{aligned}
& \theta=\frac{1}{\tilde{\Gamma}} \frac{\rho}{\rho+\rho_{g}} \frac{1}{\alpha} \frac{1}{1-\mathcal{G}} \frac{1}{\Gamma} g+\frac{1}{\tilde{\Gamma}} \Omega \frac{\rho}{\rho+v} \alpha_{v} \\
& +g \kappa(1-\xi) \frac{1}{\tilde{\Gamma}} \Omega\left[E_{2}^{\prime}\left(A+\rho_{g} I\right)^{-1} E_{1}-E_{2}^{\prime} g\left(A+\left(\rho+\rho_{g}\right) I\right)^{-1} E_{1}\right], \\
& \theta=g \frac{1}{\tilde{\Gamma}} \frac{\rho}{\rho+\rho_{g}} \frac{1}{\alpha} \frac{1}{1-\mathcal{G}} \frac{1}{\Gamma} \\
& +g \frac{1}{\tilde{\Gamma}} \Omega \frac{\rho}{\rho+v}\left[-\frac{1}{\hat{\Gamma}} \kappa(1-\xi) E_{2}^{\prime}\left(A+\rho_{g}\right)^{-1} E_{1}\right. \\
& \left.+(1-\mathcal{G})(1-\alpha)\left[1-\frac{\alpha}{1-\alpha} \lambda \hat{\sigma} \omega E_{2}^{\prime} A^{-1} E_{1}\right] \frac{\rho}{\rho+\rho_{g}} \frac{1}{\alpha} \frac{1}{1-\mathcal{G}} \frac{1}{\Gamma \tilde{\Gamma} \hat{\Gamma}}+\hat{\Omega}\right] \\
& +g \kappa(1-\xi) \frac{1}{\tilde{\Gamma}} \Omega\left[E_{2}^{\prime}\left(A+\rho_{g} I\right)^{-1} E_{1}-E_{2}^{\prime} g\left(A+\left(\rho+\rho_{g}\right) I\right)^{-1} E_{1}\right], \\
& \theta=g \frac{\rho}{\rho+\rho_{g}} \frac{1}{\alpha} \frac{1}{1-\mathcal{G}} \frac{1}{\Gamma} \frac{1}{\tilde{\Gamma}} \\
& +g \frac{1}{\tilde{\Gamma}} \Omega \frac{\rho}{\rho+v}\left[-\frac{1}{\hat{\Gamma}} \kappa(1-\xi) E_{2}^{\prime}\left(A+\rho_{g}\right)^{-1} E_{1}\right. \\
& \left.+(1-\mathcal{G})(1-\alpha)\left[1-\frac{\alpha}{1-\alpha} \lambda \hat{\sigma} \omega E_{2}^{\prime} A^{-1} E_{1}\right] \frac{\rho}{\rho+\rho_{g}} \frac{1}{\alpha} \frac{1}{1-\mathcal{G}} \frac{1}{\Gamma \tilde{\Gamma} \hat{\Gamma}}+\hat{\Omega}\right] \\
& +g \kappa(1-\xi) \frac{1}{\tilde{\Gamma}} \Omega\left[E_{2}^{\prime}\left(A+\rho_{g} I\right)^{-1} E_{1}-E_{2}^{\prime} g\left(A+\left(\rho+\rho_{g}\right) I\right)^{-1} E_{1}\right], \\
& \theta=g \frac{\rho}{\rho+\rho_{g}} \frac{1}{\alpha} \frac{1}{1-\mathcal{G}} \\
& +g \frac{\rho}{\rho+\rho_{g}} \frac{1}{\alpha} \frac{1}{1-\mathcal{G}}\left[\frac{1}{\Gamma} \frac{1}{\tilde{\Gamma}}-1\right] \\
& +g \frac{1}{\tilde{\Gamma}} \Omega \frac{\rho}{\rho+v}\left[-\frac{1}{\hat{\Gamma}} \kappa(1-\xi) E_{2}^{\prime}\left(A+\rho_{g}\right)^{-1} E_{1}\right. \\
& \left.+(1-\mathcal{G})(1-\alpha)\left[1-\frac{\alpha}{1-\alpha} \lambda \hat{\sigma} \omega E_{2}^{\prime} A^{-1} E_{1}\right] \frac{\rho}{\rho+\rho_{g}} \frac{1}{\alpha} \frac{1}{1-\mathcal{G}} \frac{1}{\Gamma \tilde{\Gamma} \hat{\Gamma}}+\hat{\Omega}\right] \\
& +g \kappa(1-\xi) \frac{1}{\tilde{\Gamma}} \Omega\left[E_{2}^{\prime}\left(A+\rho_{g} I\right)^{-1} E_{1}-E_{2}^{\prime} g\left(A+\left(\rho+\rho_{g}\right) I\right)^{-1} E_{1}\right], \\
& \theta=g \frac{\rho}{\rho+\rho_{g}} \frac{1}{\alpha} \frac{1}{1-\mathcal{G}}+g \tilde{\Omega} .
\end{aligned}
$$


We can plug back into

$$
X_{t}=\alpha_{v} e^{\nu t} X_{v}+(1-\mathcal{G}) \lambda \hat{\sigma} \alpha \omega \theta A^{-1} E_{1}+\kappa(1-\xi) \int_{t}^{\infty} g_{s} e^{-A(s-t)} E_{1} d s .
$$

We get

$$
c_{t}=\alpha_{\nu} e^{\nu t}+(1-\mathcal{G}) \lambda \hat{\sigma} \alpha \omega \theta E_{2}^{\prime} A^{-1} E_{1}+g e^{-\rho_{g} t} \kappa(1-\xi) E_{2}^{\prime}\left(A+\rho_{g} I\right)^{-1} E_{1},
$$

which we can rewrite as

$$
\begin{aligned}
& c_{t}=g \kappa(1-\xi) E_{2}^{\prime}\left(A+\rho_{g} I\right)^{-1} E_{1} e^{\nu t}\left(1-e^{-\left(\nu+\rho_{g}\right) t}\right) \\
&+g(1-\mathcal{G})(1-\alpha)\left[e^{\nu t}+\left(1-e^{\nu t}\right) \frac{\alpha}{1-\alpha} \lambda \hat{\sigma} \omega E_{2}^{\prime} A^{-1} E_{1}\right] \frac{\rho}{\rho+\rho_{g}} \frac{1}{\alpha} \frac{1}{1-\mathcal{G}} \\
&+g \tilde{\Omega}(1-\mathcal{G}) \lambda \hat{\sigma} \alpha \omega E_{2}^{\prime} A^{-1} E_{1} \\
&+g\left[\frac{1}{\Gamma \tilde{\Gamma} \hat{\Gamma}}-1\right](1-\mathcal{G})(1-\alpha)\left[1-\frac{\alpha}{1-\alpha} \lambda \hat{\sigma} \omega E_{2}^{\prime} A^{-1} E_{1}\right] \frac{\rho}{\rho+\rho_{g}} \frac{1}{\alpha} \frac{1}{1-\mathcal{G}} e^{\nu t} \\
&+g \hat{\Omega} e^{\nu t}-g\left(\frac{1}{\hat{\Gamma}}-1\right) \kappa(1-\xi) E_{2}^{\prime}\left(A+\rho_{g}\right)^{-1} E_{1} e^{v t},
\end{aligned}
$$

or

$$
\begin{aligned}
c_{t}=g \kappa(1-\xi) E_{2}^{\prime}\left(A+\rho_{g} I\right)^{-1} E_{1} e^{\nu t}\left(1-e^{-\left(\nu+\rho_{g}\right) t}\right) & \\
& +g(1-\mathcal{G})(1-\alpha)\left[e^{\nu t}+\left(1-e^{\nu t}\right) \frac{\alpha}{1-\alpha} \lambda \hat{\sigma} \omega E_{2}^{\prime} A^{-1} E_{1}\right] \frac{\rho}{\rho+\rho_{g}} \frac{1}{\alpha} \frac{1}{1-\mathcal{G}} \\
& +g \tilde{\Omega}(1-\mathcal{G}) \lambda \hat{\sigma} \alpha \omega E_{2}^{\prime} A^{-1} E_{1}+g e^{\nu t} \overline{\bar{\Omega}} .
\end{aligned}
$$

\section{H Proof of Proposition 7}

With active monetary policy, we have $i_{t}^{*}-\rho=-\hat{\sigma}(1-\xi) \dot{g}_{t}^{*}$ and $c_{t}^{*}=-(1-\xi) g_{t}^{*}$. With complete markets, $\theta=0$. The system is

$$
\begin{gathered}
\dot{\pi}_{H, t}=\rho \pi_{H, t}-\kappa c_{t}+\lambda \hat{\sigma} \alpha(\omega-1)(1-\xi) g_{t}^{*}, \\
\dot{c}_{t}=-\hat{\sigma}^{-1} \pi_{H, t}-(1-\alpha(\omega-1))(1-\xi) \dot{g}_{t}^{*}, \\
c_{0}=-(1-\xi) g_{0}^{*} .
\end{gathered}
$$


The solution is

$X_{t}=\alpha_{v} e^{\nu t} X_{v}-\lambda \hat{\sigma} \alpha(\omega-1)(1-\xi) \int_{t}^{\infty} g_{s}^{*} e^{-A(s-t)} E_{1} d s+(1-\alpha(\omega-1))(1-\xi) \int_{t}^{\infty} \dot{g}_{s}^{*} e^{-A(s-t)} E_{2} d s$,

or

$$
\begin{aligned}
X_{t}=\alpha_{v} e^{\nu t} & X_{v}-\lambda \hat{\sigma} \alpha(\omega-1)(1-\xi) \int_{t}^{\infty} g_{s}^{*} e^{-A(s-t)} E_{1} d s \\
& -(1-\alpha(\omega-1))(1-\xi) g_{t}^{*} E_{2}+(1-\alpha(\omega-1))(1-\xi) \int_{t}^{\infty} g_{s}^{*} A e^{-A(s-t)} E_{2} d s,
\end{aligned}
$$

where $X_{0}$ and $\alpha_{v}$ solve the system of three equations in three unknowns

$$
\begin{gathered}
X_{0}+\lambda \hat{\sigma} \alpha(\omega-1)(1-\xi) \int_{0}^{\infty} g_{s}^{*} e^{-A s} E_{1} d s+(1-\alpha(\omega-1))(1-\xi) g_{0}^{*} E_{2} \\
-(1-\alpha(\omega-1))(1-\xi) \int_{0}^{\infty} g_{s}^{*} A e^{-A s} E_{2} d s=\alpha_{v} X_{v}, \\
E_{2}^{\prime} X_{0}=-(1-\xi) g_{0}^{*} .
\end{gathered}
$$

We get

$$
\begin{aligned}
-\alpha(\omega-1)(1-\xi) g_{0}^{*}+\lambda \hat{\sigma} \alpha(\omega-1) & (1-\xi) \int_{0}^{\infty} g_{s}^{*} E_{2}^{\prime} e^{-A s} E_{1} d s \\
& -(1-\alpha(\omega-1))(1-\xi) \int_{0}^{\infty} g_{s}^{*} A e^{-A s} E_{2} d s=\alpha_{v},
\end{aligned}
$$

and

$$
\begin{aligned}
& c_{t}=-e^{\nu t} \alpha(\omega-1)(1-\xi) g_{0}^{*}-(1-\alpha(\omega-1))(1-\xi) g_{t}^{*} \\
& \quad+e^{\nu t} \lambda \hat{\sigma} \alpha(\omega-1)(1-\xi) \int_{0}^{\infty} g_{s}^{*} E_{2}^{\prime} e^{-A s} E_{1} d s-\lambda \hat{\sigma} \alpha(\omega-1)(1-\xi) \int_{t}^{\infty} g_{s}^{*} E_{2}^{\prime} e^{-A(s-t)} E_{1} d s \\
& -e^{\nu t}(1-\alpha(\omega-1))(1-\xi) \int_{0}^{\infty} g_{s}^{*} E_{2}^{\prime} A e^{-A s} E_{2} d s+(1-\alpha(\omega-1))(1-\xi) \int_{t}^{\infty} g_{s}^{*} E_{2}^{\prime} A e^{-A(s-t)} E_{2} d s .
\end{aligned}
$$

Using $E_{1}=\frac{1}{(\bar{v}-v) \hat{\sigma}}\left(X_{v}-X_{\bar{v}}\right)$ and $E_{2}=\frac{1}{(\bar{v}-v)}\left(\bar{v} X_{v}-v X_{\bar{v}}\right)$, we can rewrite this as

$$
c_{t}=-e^{v t} \alpha(\omega-1)(1-\xi) g_{0}^{*}-(1-\alpha(\omega-1))(1-\xi) g_{t}^{*}
$$




$$
\begin{aligned}
& +e^{v t} \lambda \hat{\sigma} \alpha(\omega-1)(1-\xi) \int_{0}^{\infty} g_{s}^{*} E_{2}^{\prime} e^{-A s} \frac{1}{(\bar{v}-v) \hat{\sigma}}\left(X_{v}-X_{\bar{v}}\right) d s \\
& -\lambda \hat{\sigma} \alpha(\omega-1)(1-\xi) \int_{t}^{\infty} g_{s}^{*} E_{2}^{\prime} e^{-A(s-t)} \frac{1}{(\bar{v}-v) \hat{\sigma}}\left(X_{v}-X_{\bar{v}}\right) d s \\
& -e^{v t}(1-\alpha(\omega-1))(1-\xi) \int_{0}^{\infty} g_{s}^{*} E_{2}^{\prime} A e^{-A s} \frac{1}{(\bar{v}-v)}\left(\bar{v} X_{v}-v X_{\bar{v}}\right) d s \\
& +(1-\alpha(\omega-1))(1-\xi) \int_{t}^{\infty} g_{s}^{*} E_{2}^{\prime} A e^{-A(s-t)} \frac{1}{(\bar{v}-v)}\left(\bar{v} X_{v}-v X_{\bar{v}}\right) d s,
\end{aligned}
$$

or

$$
\begin{aligned}
c_{t}=-e^{v t} \alpha(\omega-1) & (1-\xi) g_{0}^{*}-(1-\alpha(\omega-1))(1-\xi) g_{t}^{*} \\
& +e^{v t} \lambda \hat{\sigma} \alpha(\omega-1)(1-\xi) \int_{0}^{\infty} g_{s}^{*} \frac{1}{\hat{\sigma}} \frac{e^{-v s}-e^{-\bar{v} s}}{\bar{v}-v} d s \\
- & \lambda \hat{\sigma} \alpha(\omega-1)(1-\xi) \int_{t}^{\infty} g_{s}^{*} \frac{1}{\hat{\sigma}} \frac{e^{-v(s-t)}-e^{-\bar{v}(s-t)}}{\bar{v}-v} d s \\
- & e^{v t}(1-\alpha(\omega-1))(1-\xi) \int_{0}^{\infty} g_{s}^{*} \bar{v} v \frac{e^{-v s}-e^{-\bar{v} s}}{\bar{v}-v} d s \\
& +(1-\alpha(\omega-1))(1-\xi) \int_{t}^{\infty} g_{s}^{*} \bar{v} v \frac{e^{-v(s-t)}-e^{-\bar{v}(s-t)}}{\bar{v}-v} d s,
\end{aligned}
$$

or

$$
\begin{aligned}
& c_{t}=-e^{v t} \alpha(\omega-1)(1-\xi) g_{0}^{*}-(1-\alpha(\omega-1))(1-\xi) g_{t}^{*} \\
&+ e^{v t} \hat{\sigma} \frac{\alpha}{\hat{\sigma}+\phi}(\omega-1) \hat{\sigma}^{-1} \mathcal{K}(1-\xi) \int_{0}^{\infty} g_{s}^{*} \frac{e^{-v s}-e^{-\bar{v} s}}{\bar{v}-v} d s \\
&- \hat{\sigma} \frac{\alpha}{\hat{\sigma}+\phi}(\omega-1) \hat{\sigma}^{-1} \mathcal{K}(1-\xi) \int_{t}^{\infty} g_{s}^{*} \frac{e^{-v(s-t)}-e^{-\bar{v}(s-t)}}{\bar{v}-v} d s \\
& e^{v t}(1-\alpha(\omega-1)) \hat{\sigma}^{-1} \mathcal{K}(1-\xi) \int_{0}^{\infty} g_{s}^{*} \frac{e^{-v s}-e^{-\bar{v} s}}{\bar{v}-v} d s \\
&-(1-\alpha(\omega-1)) \hat{\sigma}^{-1} \mathcal{K}(1-\xi) \int_{t}^{\infty} g_{s}^{*} \frac{e^{-v(s-t)}-e^{-\bar{v}(s-t)}}{\bar{v}-v} d s,
\end{aligned}
$$

or

$$
\begin{aligned}
c_{t}=- & e^{v t} \alpha(\omega-1)(1-\xi) g_{0}^{*}-(1-\alpha(\omega-1))(1-\xi) g_{t}^{*} \\
& +e^{v t} \hat{\sigma}^{-1} \mathcal{K}(1-\xi)\left[\hat{\sigma} \frac{\alpha}{\hat{\sigma}+\phi}(\omega-1)+(1-\alpha(\omega-1))\right] \int_{0}^{\infty} g_{s}^{*} \frac{e^{-v s}-e^{-\bar{v} s}}{\bar{v}-v} d s \\
& \quad-\hat{\sigma}^{-1} \mathcal{K}(1-\xi)\left[\hat{\sigma} \frac{\alpha}{\hat{\sigma}+\phi}(\omega-1)+(1-\alpha(\omega-1))\right] \int_{t}^{\infty} g_{s}^{*} \frac{e^{-v(s-t)}-e^{-\bar{v}(s-t)}}{\bar{v}-v} d s .
\end{aligned}
$$


In the case where $g_{t}^{*}=g^{*} e^{-\rho_{g} t}$, we get

$$
\begin{gathered}
X_{t}=\alpha_{v} e^{v t} X_{v}-\lambda \hat{\sigma} \alpha(\omega-1)(1-\xi) g^{*} e^{-\rho_{g} t}\left(A+\rho_{g} I\right)^{-1} E_{1} \\
-(1-\alpha(\omega-1))(1-\xi) \rho_{g} g^{*} e^{-\rho_{g} t}\left(A+\rho_{g} I\right)^{-1} E_{2}, \\
-(1-\xi) g_{0}^{*}+\lambda \hat{\sigma} \alpha(\omega-1)(1-\xi) g^{*} E_{2}^{\prime}\left(A+\rho_{g} I\right)^{-1} E_{1} \\
+(1-\alpha(\omega-1))(1-\xi) \rho_{g} g^{*} E_{2}^{\prime}\left(A+\rho_{g} I\right)^{-1} E_{2}=\alpha_{v},
\end{gathered}
$$

so that

$$
\begin{aligned}
& \quad c_{t}=-e^{\nu t}(1-\xi) g_{0}^{*} \\
& +e^{\nu t} \lambda \hat{\sigma} \alpha(\omega-1)(1-\xi) g^{*} E_{2}^{\prime}\left(A+\rho_{g} I\right)^{-1} E_{1}-\lambda \hat{\sigma} \alpha(\omega-1)(1-\xi) g^{*} e^{-\rho_{g} t} E_{2}^{\prime}\left(A+\rho_{g} I\right)^{-1} E_{1} \\
& +e^{\nu t}(1-\alpha(\omega-1))(1-\xi) \rho_{g} g^{*} E_{2}^{\prime}\left(A+\rho_{g} I\right)^{-1} E_{2}-(1-\alpha(\omega-1))(1-\xi) \rho_{g} g^{*} e^{-\rho_{g} t} E_{2}^{\prime}\left(A+\rho_{g} I\right)^{-1} E_{2},
\end{aligned}
$$

or

$$
\begin{aligned}
& \quad c_{t}=-e^{\nu t}(1-\xi) g_{0}^{*} \\
& +e^{\nu t}\left(1-e^{-\left(v+\rho_{g}\right) t}\right)(1-\xi) g^{*}\left[\lambda \hat{\sigma} \alpha(\omega-1) E_{2}^{\prime}\left(A+\rho_{g} I\right)^{-1} E_{1}+(1-\alpha(\omega-1)) \rho_{g} E_{2}^{\prime}\left(A+\rho_{g} I\right)^{-1} E_{2}\right] .
\end{aligned}
$$

We use

$$
\begin{gathered}
\left(A+\rho_{g} I\right)^{-1}=\frac{1}{\rho_{g}\left(\rho+\rho_{g}\right)-\kappa \hat{\sigma}^{-1}}\left[\begin{array}{cc}
\rho_{g} & \kappa \\
\hat{\sigma}^{-1} & \rho+\rho_{g}
\end{array}\right], \\
E_{2}^{\prime}\left(A+\rho_{g} I\right)^{-1} E_{1}=\frac{\hat{\sigma}^{-1}}{\rho_{g}\left(\rho+\rho_{g}\right)-\kappa \hat{\sigma}^{-1}}, \\
E_{2}^{\prime}\left(A+\rho_{g} I\right)^{-1} E_{2}=\frac{\rho+\rho_{g}}{\rho_{g}\left(\rho+\rho_{g}\right)-\kappa \hat{\sigma}^{-1}},
\end{gathered}
$$

to get

$$
c_{t}=-e^{\nu t}(1-\xi) g_{0}^{*}+e^{\nu t}\left(1-e^{-\left(v+\rho_{g}\right) t}\right)(1-\xi) g^{*} \frac{\lambda \alpha(\omega-1)+(1-\alpha(\omega-1)) \rho_{g}\left(\rho+\rho_{g}\right)}{\rho_{g}\left(\rho+\rho_{g}\right)-\kappa \hat{\sigma}^{-1}} .
$$

In the Cole-Obstfeld case with $g_{t}^{*}=g^{*} e^{-\rho_{g} t}$, the solution is simple. We get

$$
X_{t}=\alpha_{v} e^{v t} X_{v}-e^{-\rho_{g} t}(1-\xi) \rho_{g} g^{*}\left(A+\rho_{g} I\right)^{-1} E_{2}
$$


where $X_{0}$ and $\alpha_{v}$ solve the system of three equations in three unknowns

$$
\begin{gathered}
X_{0}+(1-\xi) \rho_{g} g^{*}\left(A+\rho_{g} I\right)^{-1} E_{2}=\alpha_{v} X_{v}, \\
E_{2}^{\prime} X_{0}=-(1-\xi) g^{*} .
\end{gathered}
$$

We get

$$
\alpha_{v}=(1-\xi) g^{*}\left[\frac{\rho_{g}\left(\rho+\rho_{g}\right)}{\rho_{g}\left(\rho+\rho_{g}\right)-\kappa(1-\mathcal{G})}-1\right]
$$

and hence

$$
c_{t}=-e^{\nu t}(1-\xi) g^{*}\left[1-\left(1-e^{-\left(\rho_{g}+v\right) t}\right) \frac{\rho_{g}\left(\rho+\rho_{g}\right)}{\rho_{g}\left(\rho+\rho_{g}\right)-\kappa(1-\mathcal{G})}\right] .
$$

\section{Derivation of the Loglinearization in Section 7.1}

We have the following aggregation equations:

$$
\begin{aligned}
& c_{t}=\chi c_{t}^{r}+(1-\chi) c_{t}^{o}, \\
& n_{t}=\chi n_{t}^{r}+(1-\chi) n_{t}^{o} .
\end{aligned}
$$

We have the following conditions characterizing the solution of the agents' problems:

$$
\begin{gathered}
\dot{c}_{t}^{o}=(1-\mathcal{G}) \sigma^{-1}\left(i_{t}-\bar{r}_{t}-\pi_{t}\right), \\
c_{t}^{r}=\frac{W N^{r}}{Y}\left(w_{t}+n_{t}^{r}\right)-t_{t}^{r}, \\
w_{t}=\frac{\sigma}{1-\mathcal{G}} c_{t}^{r}+\phi n_{t}^{r} . \\
w_{t}=\frac{\sigma}{1-\mathcal{G}} c_{t}+\phi n_{t},
\end{gathered}
$$

where $w_{t}$ denotes real wages.

Combining and re-arranging, we get

$$
\begin{gathered}
n_{t}^{r}=\phi^{-1}\left(w_{t}-\frac{\sigma}{1-\mathcal{G}} c_{t}^{r}\right), \\
c_{t}^{r}=\frac{W N^{r}}{Y}\left[\left(1+\phi^{-1}\right)\left(\frac{\sigma}{1-\mathcal{G}} c_{t}+\phi n_{t}\right)-\phi^{-1} \frac{\sigma}{1-\mathcal{G}} c_{t}^{r}\right]-t_{t}^{r},
\end{gathered}
$$




$$
\begin{gathered}
c_{t}^{r}=\frac{\frac{W N^{r}}{Y}\left(1+\phi^{-1}\right)\left(\frac{\sigma}{1-\mathcal{G}} c_{t}+\phi n_{t}\right)-t_{t}^{r}}{1+\phi^{-1} \frac{\sigma}{1-\mathcal{G}} \frac{W N^{r}}{Y}}, \\
c_{t}\left[\frac{1-\chi \frac{W N^{r}}{Y} \frac{\sigma}{1-\mathcal{G}}+(1-\chi) \phi^{-1} \frac{\sigma}{1-\mathcal{G}} \frac{W N^{r}}{Y}}{1+\phi^{-1} \frac{\sigma}{1-\mathcal{G}} \frac{W N^{r}}{Y}}\right]=\chi \frac{\frac{W N^{r}}{Y}\left(1+\phi^{-1}\right) \phi n_{t}-t_{t}^{r}}{1+\phi^{-1} \frac{\sigma}{1-\mathcal{G}} \frac{W N^{r}}{Y}}+(1-\chi) c_{t}^{o}, \\
c_{t}=\chi \frac{\frac{W N^{r}}{Y}\left(1+\phi^{-1}\right) \phi n_{t}-t_{t}^{r}}{1-\chi \frac{W N^{r}}{Y} \frac{\sigma}{1-\mathcal{G}}+(1-\chi) \phi^{-1} \frac{\sigma}{1-\mathcal{G}} \frac{W N^{r}}{Y}}+(1-\chi) \frac{1+\phi^{-1} \frac{\sigma}{1-\mathcal{G}} \frac{W N^{r}}{Y}}{1-\chi \frac{W N^{r}}{Y} \frac{\sigma}{1-\mathcal{G}}+(1-\chi) \phi^{-1} \frac{\sigma}{1-\mathcal{G}} \frac{W N^{r}}{Y}} c_{t}^{o}, \\
c_{t}=\chi \frac{\phi(1+\phi) n_{t}-\frac{Y}{W N^{r}} \phi t_{t}^{r}}{\frac{Y}{W N^{r}} \phi-\chi \frac{\sigma}{1-\mathcal{G}} \phi+(1-\chi) \frac{\sigma}{1-\mathcal{G}}}+(1-\chi) \frac{\frac{Y}{W N^{r}} \phi+\frac{\sigma}{1-\mathcal{G}}}{\frac{\gamma}{W N^{r}} \phi-\chi \frac{\sigma}{1-\mathcal{G}} \phi+(1-\chi) \frac{\sigma}{1-\mathcal{G}}} c_{t}^{o}, \\
c_{t}=\chi(1-\mathcal{G}) \frac{\phi(1+\phi) n_{t}-\mu \phi t_{t}^{r}}{(1-\mathcal{G}) \mu \phi+\sigma-\chi \sigma(1+\phi)}+(1-\chi) \frac{(1-\mathcal{G}) \mu \phi+\sigma}{(1-\mathcal{G}) \mu \phi+\sigma-\chi \sigma(1+\phi)} c_{t}^{o},
\end{gathered}
$$

and finally

$$
c_{t}=\Theta_{n} n_{t}-\Theta_{\tau} t_{t}^{r}+\bar{\sigma}^{-1} \sigma \frac{1}{1-\mathcal{G}} c_{t}^{o},
$$

where

$$
\begin{gathered}
\bar{\sigma}^{-1}=\sigma^{-1}(1-\chi)(1-\mathcal{G}) \frac{(1-\mathcal{G}) \mu \phi+\sigma}{\phi(1-\mathcal{G}) \mu+\sigma-\chi \sigma(1+\phi)}, \\
\Theta_{n}=\chi(1-\mathcal{G}) \frac{(1+\phi) \phi}{\phi(1-\mathcal{G}) \mu+\sigma-\chi \sigma(1+\phi)}, \\
\Theta_{\tau}=\chi(1-\mathcal{G}) \frac{\mu \phi}{\phi(1-\mathcal{G}) \mu+\sigma-\chi \sigma(1+\phi)} .
\end{gathered}
$$

Differentiating, we get

$$
c_{t}=\Theta_{n} \dot{n}_{t}-\Theta_{\tau} \dot{t}_{t}^{r}+\bar{\sigma}^{-1}\left(i_{t}-\bar{r}_{t}-\pi_{t}\right),
$$

and using $\dot{n}_{t}=\dot{c}_{t}+\dot{g}_{t}$, we find the Euler equation

$$
\dot{c}_{t}=\tilde{\sigma}^{-1}\left(i_{t}-\bar{r}_{t}-\pi_{t}\right)+\tilde{\Theta}_{n} \dot{g}_{t}-\tilde{\Theta}_{\tau} \dot{t}_{t}^{r},
$$

where

$$
\begin{aligned}
\tilde{\sigma}^{-1} & =\frac{\bar{\sigma}^{-1}}{1-\Theta_{n}}, \\
\tilde{\Theta}_{n} & =\frac{\Theta_{n}}{1-\Theta_{n}}, \\
\tilde{\Theta}_{\tau} & =\frac{\Theta_{\tau}}{1-\Theta_{n}} .
\end{aligned}
$$


The New Keynesian Philips Curve is unchanged

$$
\dot{\pi}_{t}=\rho \pi_{t}-\kappa\left[c_{t}+(1-\xi) g_{t}\right] .
$$

\section{J Proof of Proposition 13}

Let $X_{t}=\left[\pi_{t}, c_{t}\right]^{\prime}, B_{t}=\left[-\kappa(1-\xi) g_{t}, \tilde{\Theta}_{n} \dot{g}_{t}-\tilde{\Theta}_{\tau} \dot{t}_{t}^{r}\right]^{\prime}=-\kappa(1-\xi) g_{t} E_{1}+\left[\tilde{\Theta}_{n} \dot{g}_{t}-\tilde{\Theta}_{\tau} \dot{t}_{t}^{r}\right] E_{2}$ and $A=\left[\begin{array}{cc}\rho & -\kappa \\ -\tilde{\sigma}^{-1} & 0\end{array}\right]$. We have $\dot{X}_{t}=A X_{t}+B_{t}$. The matrix $A$ has one positive and one negative eigenvalue. The negative eigenvalue is given by $\tilde{v}=\frac{\rho-\sqrt{\rho^{2}+4 \kappa \tilde{\sigma}^{-1}}}{2}$ and the positive eigenvalue is given by $\tilde{v}=\frac{\rho+\sqrt{\rho^{2}+4 \kappa \tilde{\sigma}^{-1}}}{2}$. The associated eigenvectors are $X_{\tilde{v}}=$ $[-\tilde{v} \tilde{\sigma}, 1]^{\prime}$ and $X_{\tilde{v}}=[-\tilde{\tilde{v}} \tilde{\sigma}, 1]^{\prime}$. The solution is

$$
X_{t}=\alpha_{\tilde{\nu}} e^{\tilde{\nu} t} X_{\tilde{v}}+\kappa(1-\xi) \int_{t}^{\infty} g_{s} e^{-A(s-t)} E_{1} d s-\int_{t}^{\infty}\left[\tilde{\Theta}_{n} \dot{g}_{s}-\tilde{\Theta}_{\tau} \dot{t}_{s}^{r}\right] e^{-A(s-t)} E_{2} d s,
$$

where $X_{0}$ and $\alpha_{v}$ solve the system of two equations in three unknowns

$$
X_{0}-\kappa(1-\xi) \int_{0}^{\infty} g_{t} e^{-A t} E_{1} d t+\int_{0}^{\infty}\left[\tilde{\Theta}_{n} \dot{g}_{t}-\tilde{\Theta}_{\tau} \dot{t}_{t}^{r}\right] e^{-A t} E_{2} d t=\alpha_{v} X_{v}
$$

We pick the solution with $\alpha_{v}=0$, just as in the "Fiscal Multipliers" paper. We get

$$
X_{t}=\kappa(1-\xi) \int_{t}^{\infty} g_{s} e^{-A(s-t)} E_{1} d s-\int_{t}^{\infty}\left[\tilde{\Theta}_{n} \dot{g}_{s}-\tilde{\Theta}_{\tau} \dot{t}_{s}^{r}\right] e^{-A(s-t)} E_{2} d s,
$$

which we can rewrite as

$$
X_{t}=\kappa(1-\tilde{\xi}) \int_{t}^{\infty} g_{s} e^{-A(s-t)} E_{1} d s+\left[\tilde{\Theta}_{n} g_{t}-\tilde{\Theta}_{\tau} t_{t}^{r}\right] E_{2}-\int_{t}^{\infty}\left[\tilde{\Theta}_{n} g_{s}-\tilde{\Theta}_{\tau} t_{s}^{r}\right] A e^{-A(s-t)} E_{2} d s
$$

Therefore we get

$$
c_{t}=\tilde{\Theta}_{n} g_{t}+\tilde{\Theta}_{\tau} t_{t}^{r}+\kappa(1-\xi) \int_{t}^{\infty} g_{s} E_{2} e^{-A(s-t)} E_{1} d s-\int_{t}^{\infty}\left[\tilde{\Theta}_{n} g_{s}-\tilde{\Theta}_{\tau} t_{s}^{r}\right] E_{2} A e^{-A(s-t)} E_{2} d s .
$$

Using $E_{1}=\frac{1}{(\tilde{v}-\tilde{v}) \tilde{\sigma}}\left(X_{\tilde{v}}-X_{\tilde{v}}\right)$ and $E_{2}=\frac{1}{(\tilde{v}-\tilde{v})}\left(\tilde{\bar{v}} X_{\tilde{v}}-\tilde{v} X_{\bar{v}}\right)$, we can rewrite this as

$$
c_{t}=\tilde{\Theta}_{n} g_{t}-\tilde{\Theta}_{\tau} t_{t}^{r}+\int_{t}^{\infty} \kappa \tilde{\sigma}^{-1}\left[(1-\xi) g_{s}+\tilde{\Theta}_{n} g_{s}-\tilde{\Theta}_{\tau} t_{s}^{r}\right] e^{-\tilde{v}(s-t)} \frac{e^{(\tilde{v}-\tilde{v})(s-t)}-1}{\tilde{v}-\tilde{v}} d s .
$$




\section{K Derivation of the Loglinearization for the Hand-to-Mouth Economy in Section 7.2}

Assume that $c_{t}^{*}=0$ and $i_{t}^{*}=\rho$. The loglinearized equations are

$$
\begin{gathered}
c_{t}^{o}=(1-\mathcal{G}) \theta+\frac{(1-\alpha)(1-\mathcal{G})}{\sigma} s_{t}, \\
y_{t}=-\alpha(1-\chi)(1-\mathcal{G}) \theta+c_{t}+\frac{\alpha(1-\mathcal{G}) \omega}{\sigma} s_{t}+g_{t}, \\
n x_{t}=-\alpha(1-\chi)(1-\mathcal{G}) \theta+\alpha(1-\mathcal{G})\left(\frac{\omega}{\sigma}-1\right) s_{t}, \\
y_{t}=n_{t}, \\
\dot{c}_{t}^{o}=-(1-\mathcal{G}) \sigma^{-1}\left(\pi_{H, t}+\alpha \dot{s}_{t}\right), \\
c_{t}^{r}=\frac{1}{\mu}\left(w_{t}+n_{t}^{r}\right)-t_{t}^{r}, \\
\hat{c}_{t}=\chi c_{t}^{r}+(1-\chi) c_{t}^{o}, \\
n_{t}=\chi n_{t}^{r}+(1-\chi) n_{t}^{o}, \\
w_{t}=\frac{\sigma}{1-\mathcal{G}} c_{t}^{r}+\phi n_{t}^{r}, \\
w_{t}=\frac{\sigma}{1-\mathcal{G}} \hat{c}_{t}+\phi n_{t} .
\end{gathered}
$$

Note that we have denoted total consumption of home agents by $\hat{c}_{t}$ to avoid a confusion with $c_{t}$, the total consumption of home goods by private agents (both home and foreign).

We use the equations the last six equations to get

$$
\hat{c}_{t}=\Theta_{n} n_{t}-\Theta_{\tau} t_{t}^{r}+\bar{\sigma}^{-1} \sigma \frac{1}{1-\mathcal{G}} c_{t}^{o} .
$$

Next we use $n_{t}=y_{t}$ together with the goods market clearing condition to get

$$
n_{t}=-\alpha(1-\chi)(1-\mathcal{G}) \theta+\frac{\alpha(1-\mathcal{G}) \omega}{\sigma} s_{t}+\hat{c}_{t}+g_{t} .
$$

Differentiating the Backus-Smith condition, we get (we could have gotten this equation directly from the definition of $s_{t}$ )

$$
\dot{s}_{t}=-\pi_{H, t}
$$


Now get can get to an equation involving total (home + foreign) consumption of the domestic good $c_{t}=y_{t}-g_{t}$ which yields

$$
c_{t}=-\alpha(1-\chi)(1-\mathcal{G}) \theta+\hat{c}_{t}+\frac{\alpha(1-\mathcal{G}) \omega}{\sigma} s_{t} .
$$

Differentiating, we get

$$
\dot{c}_{t}=\dot{\hat{c}}_{t}+\frac{\alpha(1-\mathcal{G}) \omega}{\sigma} \dot{s}_{t}
$$

then combining with the equation for $\hat{c}_{t}$

$$
\dot{c}_{t}=\Theta_{n} \dot{n}_{t}-\Theta_{\tau} \dot{t}_{t}^{r}+\bar{\sigma}^{-1} \sigma \frac{1}{1-\mathcal{G}} \dot{c}_{t}^{o}+\frac{\alpha(1-\mathcal{G}) \omega}{\sigma} \dot{s}_{t}
$$

and replacing $n_{t}=\hat{c}_{t}+g_{t}$

$$
\dot{c}_{t}=\Theta_{n}\left(\dot{\hat{c}}_{t}+\dot{g}_{t}\right)-\Theta_{\tau} \dot{t}_{t}^{r}+\bar{\sigma}^{-1} \sigma \frac{1}{1-\mathcal{G}} \dot{c}_{t}^{o}+\frac{\alpha(1-\mathcal{G}) \omega}{\sigma} \dot{s}_{t},
$$

and rearranging

$$
\dot{c}_{t}=\tilde{\Theta}_{n} \dot{g}_{t}-\tilde{\Theta}_{\tau} \dot{t}_{t}^{r}+\tilde{\sigma}^{-1} \sigma \frac{1}{1-\mathcal{G}} \dot{c}_{t}^{o}+\frac{1}{1-\Theta_{n}} \frac{\alpha(1-\mathcal{G}) \omega}{\sigma} \dot{\boldsymbol{s}}_{t},
$$

then using the Euler equation for optimizers

$$
\dot{c}_{t}=\tilde{\Theta}_{n} \dot{g}_{t}-\tilde{\Theta}_{\tau} \dot{t}_{t}^{r}-\tilde{\sigma}^{-1} \pi_{t}+\alpha\left[-\tilde{\sigma}^{-1}+\frac{\sigma^{-1}}{1-\Theta_{n}}(1-\mathcal{G}) \omega\right] \dot{s}_{t}
$$

and finally combining with the expression for $\dot{s}_{t}=-\pi_{t}$

$$
\dot{c}_{t}=\tilde{\Theta}_{n} \dot{g}_{t}-\tilde{\Theta}_{\tau} \dot{t}_{t}^{r}-\left[\tilde{\sigma}^{-1}-\alpha \tilde{\sigma}^{-1}+\alpha \frac{\sigma^{-1}}{1-\Theta_{n}}(1-\mathcal{G}) \omega\right] \pi_{t}
$$

which we can rewrite as

$$
\begin{gathered}
\dot{c}_{t}=\tilde{\Theta}_{n} \dot{g}_{t}-\tilde{\Theta}_{\tau} \dot{t}_{t}^{r}-\frac{\bar{\sigma}^{-1}}{1-\Theta_{n}}\left[1-\alpha+\alpha \frac{\sigma^{-1}(1-\mathcal{G})}{\hat{\sigma}^{-1}} \omega\right] \pi_{t,} \\
\dot{c}_{t}=\tilde{\Theta}_{n} \dot{g}_{t}-\tilde{\Theta}_{\tau} \dot{t}_{t}^{r}-\frac{\sigma^{-1}(1-\chi)(1-\mathcal{G})}{1-\Theta_{n}}\left[(1-\alpha) \frac{(1-\mathcal{G}) \mu \phi+\sigma}{\phi(1-\mathcal{G}) \mu+\sigma-\chi \sigma(1+\phi)}+\alpha \omega\right] \pi_{t}, \\
\dot{c}_{t}=\tilde{\Theta}_{n} \dot{g}_{t}-\tilde{\Theta}_{\tau} \dot{t}_{t}^{r}-\tilde{\sigma}^{-1} \pi_{t},
\end{gathered}
$$


where

$$
\tilde{\sigma}^{-1}=\frac{\sigma^{-1}(1-\chi)(1-\mathcal{G})}{1-\Theta_{n}}\left[(1-\alpha) \frac{(1-\mathcal{G}) \mu \phi+\sigma}{\phi(1-\mathcal{G}) \mu+\sigma-\chi \sigma(1+\phi)}+\alpha \omega\right]
$$

i.e. ${ }^{15}$

$$
\tilde{\sigma}=\sigma \frac{1-\Theta_{n}}{(1-\chi)(1-\mathcal{G})} \frac{1}{(1-\alpha) \frac{(1-\mathcal{G}) \mu \phi+\sigma}{\phi(1-\mathcal{G}) \mu+\sigma-\chi \sigma(1+\phi)}+\alpha \omega} .
$$

This is our Euler equation.

To derive an initial condition, we use

$$
\begin{gathered}
\hat{c}_{t}=\Theta_{n} n_{t}-\Theta_{\tau} t_{t}^{r}+\bar{\sigma}^{-1} \sigma \frac{1}{1-\mathcal{G}} c_{t}^{o}, \\
c_{t}=-\alpha(1-\chi)(1-\mathcal{G}) \theta+\hat{c}_{t}+\frac{\alpha(1-\mathcal{G}) \omega}{\sigma} s_{t}, \\
c_{t}^{o}=(1-\mathcal{G}) \theta+\frac{(1-\alpha)(1-\mathcal{G})}{\sigma} s_{t},
\end{gathered}
$$

and

$$
n_{t}=c_{t}+g_{t}
$$

to get

$$
\left(1-\Theta_{n}\right) c_{t}=-\alpha(1-\chi)(1-\mathcal{G}) \theta+\bar{\sigma}^{-1} \sigma \theta+\Theta_{n} g_{t}-\Theta_{\tau} t_{t}^{r}+\bar{\sigma}^{-1}(1-\alpha) s_{t}+\frac{\alpha(1-\mathcal{G}) \omega}{\sigma} s_{t},
$$

and apply it at $t=0$ with $s_{0}=0$ to get

$$
c_{0}=\left[\bar{\sigma}^{-1} \frac{\sigma}{1-\Theta_{n}}-\alpha \frac{(1-\chi)(1-\mathcal{G})}{1-\Theta_{n}}\right] \theta+\tilde{\Theta}_{n} g_{0}-\tilde{\Theta}_{\tau} t_{0}^{r}
$$

Hence with complete markets, this boils down to the simple condition

$$
c_{0}=\tilde{\Theta}_{n} g_{0}-\tilde{\Theta}_{\tau} t_{0}^{r}
$$

Finally we need to compute

$$
m c_{t}=w_{t}+p_{t}-p_{H, t}=w_{t}+\alpha s_{t}
$$

\footnotetext{
${ }^{15}$ We can check that when there are no hand-to-mouth consumers, this boils down to

$$
\dot{c}_{t}=-\sigma^{-1}(1-\mathcal{G})[1+\alpha(\omega-1)] \pi_{t},
$$

which is exactly the expression that we found.
} 
We have

$$
\begin{gathered}
w_{t}=\frac{\sigma}{1-\mathcal{G}} \hat{c}_{t}+\phi n_{t}, \\
w_{t}=\frac{\sigma}{1-\mathcal{G}} \hat{c}_{t}+\phi\left(c_{t}+g_{t}\right),
\end{gathered}
$$

which using

$$
\hat{c}_{t}=c_{t}+\alpha(1-\chi)(1-\mathcal{G}) \theta-\frac{\alpha(1-\mathcal{G}) \omega}{\sigma} s_{t},
$$

we can rewrite as

$$
w_{t}=\frac{\sigma}{1-\mathcal{G}} c_{t}+\sigma \alpha(1-\chi) \theta-\alpha \omega s_{t}+\phi\left(c_{t}+g_{t}\right),
$$

so that

$$
w_{t}+\alpha s_{t}=\frac{\sigma}{1-\mathcal{G}^{C}} c_{t}+\sigma \alpha(1-\chi) \theta+\alpha(1-\omega) s_{t}+\phi\left(c_{t}+g_{t}\right),
$$

which using

$$
\left[\bar{\sigma}^{-1}(1-\alpha)+\alpha \omega \frac{(1-\mathcal{G})}{\sigma}\right] s_{t}=\left(1-\Theta_{n}\right) c_{t}+\alpha(1-\chi)(1-\mathcal{G}) \theta-\bar{\sigma}^{-1} \sigma \theta-\Theta_{n} g_{t}+\Theta_{\tau} t_{t}^{r},
$$

we can rewrite as

$$
\begin{aligned}
w_{t}+\alpha s_{t}=\left(\frac{\sigma}{1-\mathcal{G}}+\phi\right) c_{t}+\phi g_{t}+\sigma \alpha(1-\chi) \theta & \\
& +\alpha(1-\omega) \frac{\left(1-\Theta_{n}\right) c_{t}+\alpha(1-\chi)(1-\mathcal{G}) \theta-\bar{\sigma}^{-1} \sigma \theta-\Theta_{n} g_{t}+\Theta_{\tau} t_{t}^{r}}{\bar{\sigma}^{-1}(1-\alpha)+\alpha \omega \frac{(1-\mathcal{G})}{\sigma}}, \\
w_{t}+\alpha s_{t}=\left[\frac{\sigma}{1-\mathcal{G}}+\phi+\frac{\alpha(1-\omega)\left(1-\Theta_{n}\right)}{\left.\bar{\sigma}^{-1}(1-\alpha)+\alpha \omega \frac{(1-\mathcal{G})}{\sigma}\right] c_{t}}\right. & +\left[\sigma \alpha(1-\chi)+\alpha(1-\omega) \frac{\alpha(1-\chi)(1-\mathcal{G})-\bar{\sigma}^{-1} \sigma}{\bar{\sigma}^{-1}(1-\alpha)+\alpha \omega \frac{(1-\mathcal{G})}{\sigma}}\right] \theta \\
+ & +\left[\phi-\frac{\alpha(1-\omega) \Theta_{n}}{\bar{\sigma}^{-1}(1-\alpha)+\alpha \omega \frac{(1-\mathcal{G})}{\sigma}}\right] g_{t}+\frac{\alpha(1-\omega) \Theta_{\tau} t_{t}^{r}}{\bar{\sigma}^{-1}(1-\alpha)+\alpha \omega \frac{(1-\mathcal{G})}{\sigma}} .
\end{aligned}
$$

We can then replace this expression in to get the New Keynesian Philips Curve

$$
\dot{\pi}_{H, t}=\rho \pi_{H, t}-\lambda\left(w_{t}+\alpha s_{t}\right) .
$$


To summarize the system is summarized by

$$
\begin{gathered}
\dot{c}_{t}=\tilde{\Theta}_{n} \dot{g}_{t}-\tilde{\Theta}_{\tau} \dot{t}_{t}^{r}-\tilde{\sigma}^{-1} \pi_{H, t} \\
\dot{\pi}_{H, t}=\rho \pi_{H, t}-\lambda\left(w_{t}+\alpha s_{t}\right), \\
c_{0}=\left[\bar{\sigma}^{-1} \frac{\sigma}{1-\Theta_{n}}-\alpha \frac{(1-\chi)(1-\mathcal{G})}{1-\Theta_{n}}\right] \theta+\tilde{\Theta}_{n} g_{0}-\tilde{\Theta}_{\tau} t_{0}^{r},
\end{gathered}
$$

where

$$
\begin{aligned}
w_{t}+\alpha s_{t}=\left[\frac{\sigma}{1-\mathcal{G}}+\phi+\right. & \left.\frac{\alpha(1-\omega)\left(1-\Theta_{n}\right)}{\bar{\sigma}^{-1}(1-\alpha)+\alpha \omega \frac{(1-\mathcal{G})}{\sigma}}\right] c_{t} \\
+\left[\sigma \alpha(1-\chi)+\alpha(1-\omega) \frac{\alpha(1-\chi)(1-\mathcal{G})-\bar{\sigma}^{-1} \sigma}{\bar{\sigma}^{-1}(1-\alpha)+\alpha \omega \frac{(1-\mathcal{G})}{\sigma}}\right] \theta & \\
& +\left[\phi-\frac{\alpha(1-\omega) \Theta_{n}}{\bar{\sigma}^{-1}(1-\alpha)+\alpha \omega \frac{(1-\mathcal{G})}{\sigma}}\right] g_{t}+\frac{\alpha(1-\omega) \Theta_{\tau} t_{t}^{r}}{\bar{\sigma}^{-1}(1-\alpha)+\alpha \omega \frac{(1-\mathcal{G})}{\sigma}} .
\end{aligned}
$$

Complete markets (for optimizers) amounts to $\theta=0$. Define $\tilde{\alpha}$ by

$$
(1-\tilde{\alpha})(1-\mathcal{G})=\left[\bar{\sigma}^{-1} \frac{\sigma}{1-\Theta_{n}}-\alpha \frac{(1-\chi)(1-\mathcal{G})}{1-\Theta_{n}}\right] .
$$

Define

$$
\tilde{\kappa}=\frac{\sigma}{1-\mathcal{G}}+\phi+\frac{\alpha(1-\omega)\left(1-\Theta_{n}\right)}{\bar{\sigma}^{-1}(1-\alpha)+\alpha \omega \frac{(1-\mathcal{G})}{\sigma}} .
$$

Define $\tilde{\xi}$ by

$$
\tilde{\kappa}(1-\tilde{\xi})=\left[\phi-\frac{\alpha(1-\omega) \Theta_{n}}{\bar{\sigma}^{-1}(1-\alpha)+\alpha \omega \frac{(1-\mathcal{G})}{\sigma}}\right] .
$$

And define $\tilde{\omega}$ by

$$
(1-\mathcal{G}) \tilde{\sigma} \tilde{\alpha} \tilde{\omega}=\sigma \alpha(1-\chi)+\alpha(1-\omega) \frac{\alpha(1-\chi)(1-\mathcal{G})-\bar{\sigma}^{-1} \sigma}{\bar{\sigma}^{-1}(1-\alpha)+\alpha \omega \frac{(1-\mathcal{G})}{\sigma}} .
$$

Then we can rewrite the system as

$$
\begin{gathered}
\dot{\pi}_{H, t}=\rho \pi_{H, t}-\tilde{\kappa}\left(c_{t}+(1-\tilde{\xi}) g_{t}\right)-(1-\mathcal{G}) \lambda \tilde{\sigma} \tilde{\alpha} \tilde{\omega} \theta, \\
\dot{c}_{t}=-\tilde{\sigma}^{-1} \pi_{H, t}+\tilde{\Theta}_{n} \dot{g}_{t}-\tilde{\Theta}_{\tau} \dot{t}_{t}^{r},
\end{gathered}
$$


with an initial condition

$$
c_{0}=(1-\mathcal{G})(1-\tilde{\alpha}) \theta+\tilde{\Theta}_{n} g_{0}-\tilde{\Theta}_{\tau} t_{0}^{r} .
$$

\section{Proof of Proposition 14}

We treat the case where optimizers have access co complete markets. The system is

$$
\begin{gathered}
\dot{\pi}_{H, t}=\rho \pi_{H, t}-\tilde{\kappa}\left(c_{t}+(1-\tilde{\zeta}) g_{t}\right), \\
\dot{c}_{t}=-\tilde{\sigma}^{-1} \pi_{H, t}+\tilde{\Theta}_{n} \dot{g}_{t}-\tilde{\Theta}_{\tau} \dot{t}_{t}^{r}, \\
c_{0}=\tilde{\Theta} g_{0}-\tilde{\Theta}_{\tau} t_{0}^{r} .
\end{gathered}
$$

Let $X_{t}=\left[\pi_{H, t}, c_{t}\right]^{\prime}, B_{t}=\left[-\tilde{\kappa}(1-\tilde{\xi}) g_{t}, \tilde{\Theta}_{n} \dot{g}_{t}-\tilde{\Theta}_{\tau} \dot{t}_{t}^{r}\right]^{\prime}=-\tilde{\kappa}(1-\tilde{\xi}) g_{t} E_{1}+\left[\tilde{\Theta}_{n} \dot{g}_{t}-\tilde{\Theta}_{\tau} \dot{t}_{t}^{r}\right] E_{2}$ and $A=\left[\begin{array}{cc}\rho & -\tilde{\kappa} \\ -\tilde{\sigma}^{-1} & 0\end{array}\right]$. We have $\dot{X}_{t}=A X_{t}+B_{t}$. The matrix $A$ has one positive and one negative eigenvalue. The negative eigenvalue is given by $\tilde{v}=\frac{\rho-\sqrt{\rho^{2}+4 \tilde{\kappa} \tilde{\kappa}^{-1}}}{2}$ and the positive eigenvalue is given by $\tilde{v}=\frac{\rho+\sqrt{\rho^{2}+4 \tilde{\kappa} \tilde{\tilde{\sigma}}-1}}{2}$. The associated eigenvectors are $X_{\tilde{v}}=[-\tilde{v} \tilde{\sigma}, 1]^{\prime}$ and $X_{\tilde{v}}=[-\tilde{v} \tilde{\sigma}, 1]^{\prime}$. The solution is

$$
X_{t}=\alpha_{\tilde{\nu}} e^{\tilde{\nu} t} X_{\tilde{\nu}}+\tilde{\kappa}(1-\tilde{\xi}) \int_{t}^{\infty} g_{s} e^{-A(s-t)} E_{1} d s-\int_{t}^{\infty}\left[\tilde{\Theta}_{n} \dot{g}_{s}-\tilde{\Theta}_{\tau} \dot{t}_{s}^{r}\right] e^{-A(s-t)} E_{2} d s,
$$

which we can rewrite as

$X_{t}=\alpha_{\tilde{\nu}} e^{\tilde{e} t} X_{\tilde{v}}+\tilde{\kappa}(1-\xi) \int_{t}^{\infty} g_{s} e^{-A(s-t)} E_{1} d s+\left[\tilde{\Theta}_{n} g_{t}-\tilde{\Theta}_{\tau} t_{t}^{r}\right] E_{2}-\int_{t}^{\infty}\left[\tilde{\Theta}_{n} g_{s}-\tilde{\Theta}_{\tau} t_{s}^{r}\right] A e^{-A(s-t)} E_{2} d s$,

where $X_{0}$ and $\alpha_{\tilde{v}}$ solve the system of three equations in three unknowns

$$
\begin{gathered}
X_{0}-\tilde{\kappa}(1-\tilde{\xi}) \int_{0}^{\infty} g_{t} e^{-A t} E_{1} d t-\left[\tilde{\Theta}_{n} g_{t}-\tilde{\Theta}_{\tau} t_{t}^{r}\right] E_{2}+\int_{0}^{\infty}\left[\tilde{\Theta}_{n} g_{t}-\tilde{\Theta}_{\tau} t_{t}^{r}\right] A e^{-A t} E_{2} d t=\alpha_{\tilde{v}} X_{\tilde{v}}, \\
E_{2}^{\prime} X_{0}=\tilde{\Theta} g_{0}-\tilde{\Theta}_{\tau} t_{0}^{r} .
\end{gathered}
$$

This yields

$$
-\tilde{\kappa}(1-\xi) \int_{0}^{\infty} g_{t} e^{-A t} E_{1} d t+\int_{0}^{\infty}\left[\tilde{\Theta}_{n} g_{t}-\tilde{\Theta}_{\tau} t_{t}^{r}\right] A e^{-A t} E_{2} d t=\alpha_{\tilde{v}} .
$$


We therefore have

$$
\begin{aligned}
& c_{t}=\left[-\tilde{\kappa}(1-\tilde{\xi}) \int_{0}^{\infty} g_{t} e^{-A t} E_{1} d t+\int_{0}^{\infty}\left[\tilde{\Theta}_{n} g_{t}-\tilde{\Theta}_{\tau} t_{t}^{r}\right] A e^{-A t} E_{2} d t\right] e^{\tilde{\nu} t} \\
& +\tilde{\kappa}(1-\xi) \int_{t}^{\infty} g_{s} E_{2}^{\prime} e^{-A(s-t)} E_{1} d s-\int_{t}^{\infty}\left[\tilde{\Theta}_{n} \dot{g}_{s}-\tilde{\Theta}_{\tau} \dot{t}_{s}^{r}\right] E_{2}^{\prime} e^{-A(s-t)} E_{2} d s, \\
& c_{t}=\left[-\tilde{\kappa}(1-\tilde{\xi}) \int_{0}^{\infty} g_{t} e^{-A t} E_{1} d t+\int_{0}^{\infty}\left[\tilde{\Theta}_{n} g_{t}-\tilde{\Theta}_{\tau} t_{t}^{r}\right] A e^{-A t} E_{2} d t\right] e^{\tilde{\nu} t} \\
& +\tilde{\kappa}(1-\tilde{\xi}) \int_{t}^{\infty} g_{s} E_{2}^{\prime} e^{-A(s-t)} E_{1} d s+\left[\tilde{\Theta}_{n} g_{t}-\tilde{\Theta}_{\tau} t_{t}^{r}\right]-\int_{t}^{\infty}\left[\tilde{\Theta}_{n} g_{s}-\tilde{\Theta}_{\tau} t_{s}^{r}\right] E_{2}^{\prime} A e^{-A(s-t)} E_{2} d s, \\
& c_{t}=\tilde{\Theta}_{n} g_{t}-\tilde{\Theta}_{\tau} t_{t}^{r}+ \\
& {\left[-\tilde{\kappa}(1-\tilde{\xi}) \int_{0}^{\infty} g_{t} e^{-A t} E_{1} d t+\int_{0}^{\infty}\left[\tilde{\Theta}_{n} g_{t}-\tilde{\Theta}_{\tau} t_{t}^{r}\right] A e^{-A t} E_{2} d t\right] e^{v t}} \\
& +\tilde{\kappa}(1-\xi) \int_{t}^{\infty} g_{s} E_{2}^{\prime} e^{-A(s-t)} E_{1} d s-\int_{t}^{\infty}\left[\tilde{\Theta}_{n} g_{s}-\tilde{\Theta}_{\tau} t_{s}^{r}\right] E_{2}^{\prime} A e^{-A(s-t)} E_{2} d s, \\
& c_{t}=\tilde{\Theta}_{n} g_{t}-\tilde{\Theta}_{\tau} t_{t}^{r}+ \\
& {\left[-\int_{0}^{\infty} \tilde{\kappa} \tilde{\sigma}^{-1}\left[(1-\tilde{\xi}) g_{s}+\tilde{\Theta}_{n} g_{s}-\tilde{\Theta}_{\tau} t_{s}^{r}\right] e^{-\tilde{v} s} \frac{e^{(\tilde{v}-\tilde{v}) s}-1}{\tilde{v}-\tilde{v}} d s\right] e^{\tilde{\nu} t}} \\
& +\int_{t}^{\infty} \tilde{\kappa} \tilde{\sigma}^{-1}\left[(1-\xi) g_{s}+\tilde{\Theta}_{n} g_{s}-\tilde{\Theta}_{\tau} t_{s}^{r}\right] e^{-\tilde{v}(s-t)} \frac{e^{(\tilde{v}-\tilde{v})(s-t)}-1}{\tilde{v}-\tilde{v}} d s .
\end{aligned}
$$

